\title{
Numerische Untersuchung zur instationären Kutta-Bedingung
}

\author{
Dissertation \\ zur Erlangung des Doktorgrades \\ der Mathematisch-Naturwissenschaftlichen Fakultäten \\ der Georg-August-Universität zu Göttingen
}

vorgelegt von

Guido van Bebber

aus

Kleve

Göttingen 2000 
D7

Referent: Prof. Dr. D. Ronneberger Korreferent: Prof. Dr. H. Eckelmann

Tag der mündlichen Prüfung: 20. Juni 2000 


\section{Inhaltsverzeichnis}

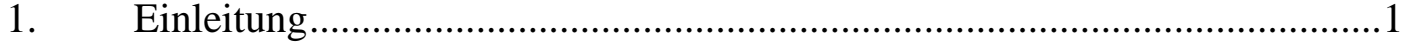

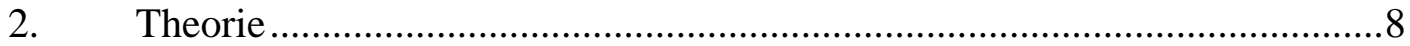

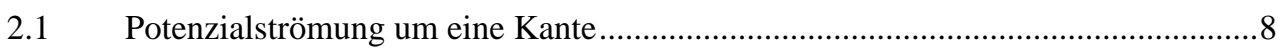

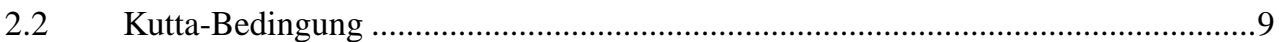

2.2.1 Definition und Historie .............................................................................. 10

2.2.2 Gültigkeit für instationäre Strömungen .....................................................11

2.3 Modell der instationären Strömungsablösung .......................................................11

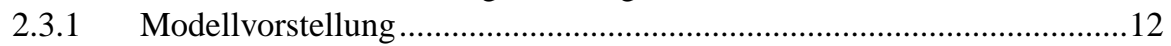

2.3.2 Zeitskalen des Modells .............................................................................14

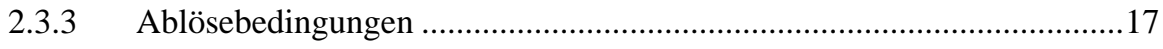

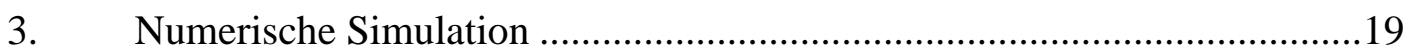

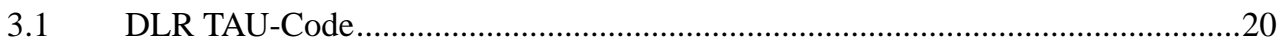

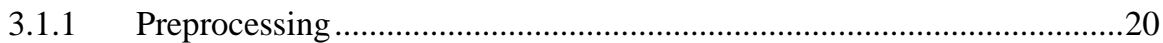

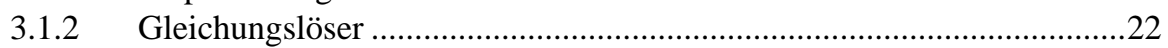

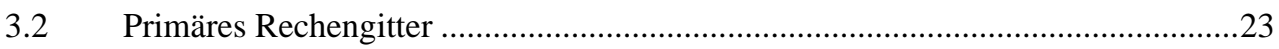

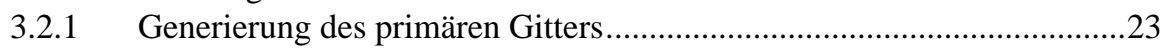

3.2.2 Größe der Einströmränder ..................................................................25

3.2.3 Anforderungen der Einströmrandbedingungen an das Gitter..................26

3.2.4 Vorlauf der Strömung bis zur Kante ......................................................26

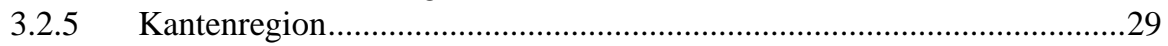

3.3 Berechnung der Wirbelstärke am Rand .............................................................

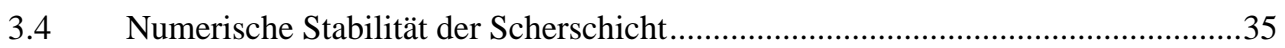

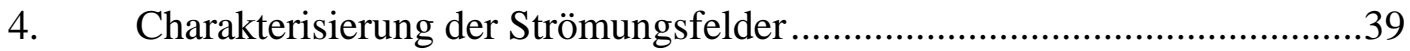

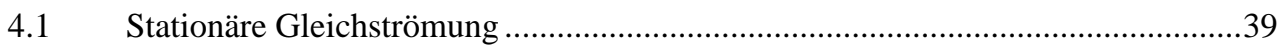

4.1.1 Stationäre Einströmrandbedingungen......................................................40

4.1.2 Eigenschaften der Gleichströmung ......................................................42

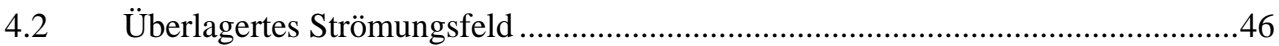

4.2.1 Instationäre Einströmrandbedingung ......................................................46

4.2.2 Charakterisierung der Anregung...........................................................4

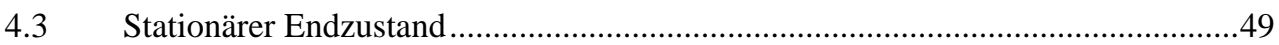

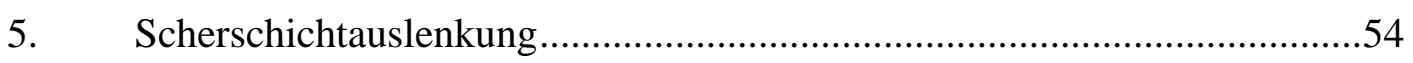

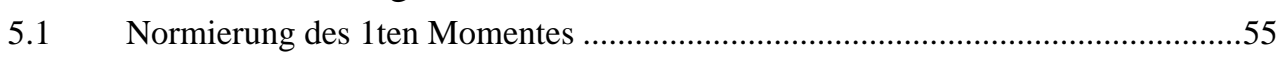

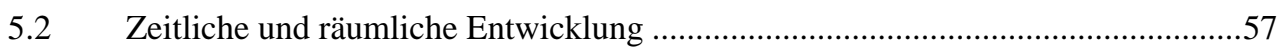

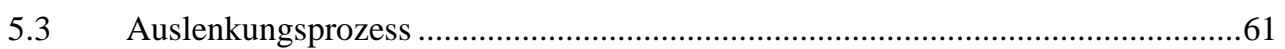

5.3.1 Abhängigkeit der maximalen Auslenkung vom Messort .........................61

5.3.2 Effekte in der Kantenregion...................................................................63

5.3.3 Zeitliche Entwicklung der maximalen Auslenkung ...................................64

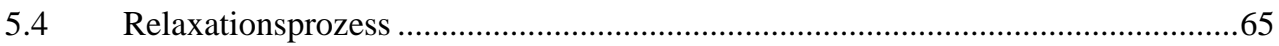

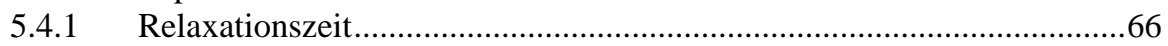

5.4.2 Phasen der Relaxation.......................................................................66

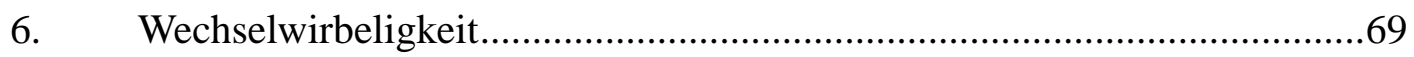

6.1 Auslenkung der Scherschicht .........................................................................

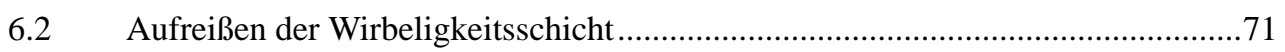

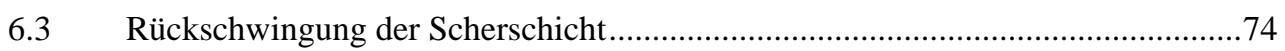

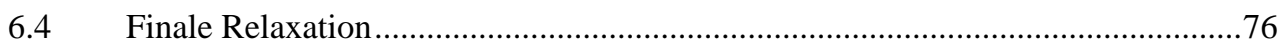

7. Zusammenfassung, Schlussfolgerungen und Ausblick ...............................78

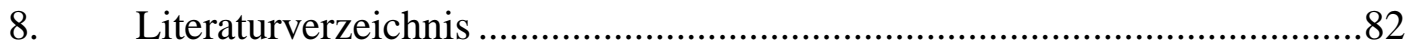




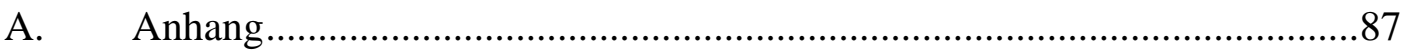

A.1 Charakterisierende strömungsphysikalische Größen ......................................87

A.2 Validierung nach der DFG-Benchmarkstudie .............................................. 89

A.2.1 Allgemeine Spezifikationen .......................................................... 90

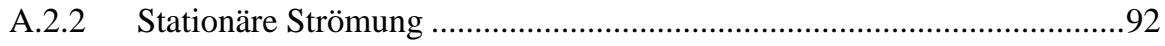

A.2.3 Instationäre Strömung...................................................................93 


\section{Symbolverzeichnis}

\section{Griechische Bezeichnungen}

$\delta_{\text {mom }}(\mathrm{x})$ : zweites Moment der Wirbeligkeitsverteilung

$\gamma$ : charakteristische Länge der Scherschichtauslenkung

$\Theta$ : Impulsverlustdicke

$v$ : kinematische Viskosität

$\xi$ : dimensionsloser Parameter zur Bestimmung der Geschwindigkeit an der Wand

$\rho$ : Dichte

$\tau^{\mathrm{i}}: \tau^{\mathrm{i}}$-Code ist die Bezeichnung für den DLR TAU-Code für inkompressible Fluide

$\omega$ : Wirbeligkeit

$\Omega_{\text {mom }}(\mathrm{x})$ : nulltes Moment der Wirbeligkeitsverteilung

\section{Lateinische Bezeichnungen}

a : halbe Kanalhöhe

A : Amplitude der Potenzialströmung

H : Höhe der Einströmränder

$\mathrm{P}_{\mathrm{b}}:$ Randpunkt des Gitters

$\mathrm{P}_{\mathrm{i}}$ : Hilfspunkt bei der Berechnung der Wirbelstärke am Rand des Gitters

$\mathrm{P}_{0}$ : zusätzlicher Punkt, entstanden durch Verschiebung eines Randpunktes

$\operatorname{Re}_{\Theta}:$ Reynoldszahl, gebildet mit der Impulsverlustdicke

$\mathrm{St}_{\Theta}:$ Strouhalzahl, gebildet mit der Impulsverlustdicke

$\mathrm{t}:$ Zeitpunkt 
$\mathrm{T}_{\text {diff }}:$ Diffusionszeit

$\mathrm{T}_{\text {konv }}:$ Konvektionszeit

$\mathrm{u}:$ Strömungsgeschwindigkeit in $\mathrm{x}-$ Richtung

$\overrightarrow{\mathrm{u}}$ : Vektor der Strömungsgeschwindigkeit

$\mathrm{u}_{\mathrm{n}}$ : Strömungsgeschwindigkeit normal zur Wand

$\mathrm{u}_{\mathrm{t}}:$ Strömungsgeschwindigkeit tangential zur Wand

$\mathrm{U}_{0}$ : von y unabhängige Geschwindigkeit am Kanaleintritt

$\mathrm{U}_{\infty}$ : Geschwindigkeit der freien Strömung

$\mathrm{U}_{\Delta}$ : Geschwindigkeit der überlagerten Potenzialströmung

$\mathrm{v}:$ Strömungsgeschwindigkeit in y-Richtung

$\mathrm{v}_{\Delta}: \mathrm{v}$-Komponente der überlagerten Potenzialströmung

$\mathrm{Y}_{\text {mom }}(\mathrm{x})$ : erstes Moment der Wirbeligkeitsverteilung 


\section{Einleitung}

Bei vielen aerodynamischen und aeroakustischen Fragestellungen ist die Dynamik der instationären Scherschichtablösung von Bedeutung. Dieser Prozess tritt immer dort auf, wo eine instationäre Strömungsgrenzschicht von einer Körperoberfläche ablöst und in eine freie Scherschicht übergeht. Dies ist z.B. der Fall bei der Ablösung der Strömung von Tragflügeln und Rotorblättern oder beim Austritt des Strahls aus einem Triebwerk, aber auch bei der Überströmung von Kavitäten, wie Fahrwerkschächten bei Flugzeugen oder Dachfenstern bei Straßenfahrzeugen.

Vielfach ist die Dynamik der Strömung unmittelbar an der Kante maßgeblich für die Erzeugung und Streuung von Schall oder ggf. auch für die Selbstanregung von rückgekoppelten Schwingungen. Besonders im Ablösebereich beeinflussen äußere Störungen die Eigendynamik der Scherschicht, so dass z.B. nachfolgend eine räumlich anwachsende Instabilität (z.B. Kelvin-Helmholtz-Instabilität) entstehen kann.

Zur Beschreibung der instationären Strömungsablösung ist die Annahme einer geeigneten Ablösebedingung grundlegend. In der Literatur finden sich hierzu verschiedene theoretische Beiträge, die jeweils von der Abflussbedingung einer stationären Strömung, der sog. Kutta-Bedingung, ausgehen und diese u.a. zu einer quasistationären Kutta-Bedingung für instationäre Strömungen erweitern.

Ziel dieser Arbeit ist die numerische Untersuchung der instationären Kutta-Bedingung. Diese wird für die Strömungsablösung an der Hinterkante einer Splitterplatte analysiert. Die Grenzschicht der Hauptströmung oberhalb der Platte geht an deren Hinterkante in eine freie Scherschicht über, die sich als Wirbeligkeitsschicht beschreiben läßt. Unterhalb der Platte strömt das Fluid mit ca. 10\% der Hauptströmungsgeschwindigkeit. Dieser Bereich wird nachfolgend als Spülströmung bezeichnet. Die Anregung dieser stationären Grundströmung erfolgt durch einen Druckgradientenimpuls. Dies entspricht der Überlagerung eines Potenzialfeldes, welches zu einer Umströmung der Hinterkante und somit zur Verletzung der Kutta-Bedingung führt (s. Abbildung 1.1).

Die Auslenkung der Strömung ist mit der Erzeugung zusätzlicher Wirbeligkeit verbunden. Erst nach deren Umverteilung ist die Kutta-Bedingung wieder erfüllt. Die an dieser Relaxation der Strömung beteiligten Prozesse und deren Wechselwirkungen sind bisher nicht vollständig bekannt. Diese Arbeit soll darüber weiteren Aufschluss geben. 

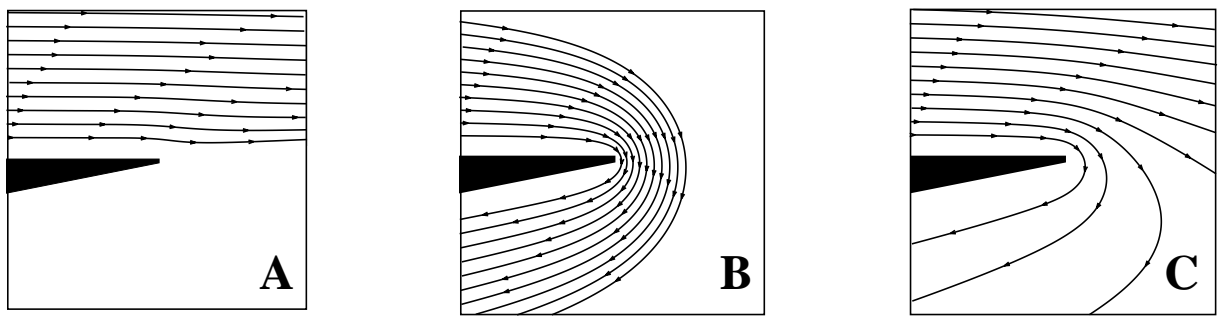

Abbildung 1.1: Prozess der instationären Scherschichtauslenkung: Einer stationär ablösenden Strömung (A) wird ein Potenzialfeld (B) überlagert. Dies führt zur Verletzung der Kutta-Bedingung; die Strömung (C) löst nicht mehr tangential ab.

Die gewonnenen Ergebnisse werden anhand eines in [Graf (1998)] vorgestellten Wirbeltransportmodells interpretiert. Die Wirbeligkeit wird dort als passive, nicht mit der Strömung wechselwirkende Substanz, betrachtet. Es wird die Frage aufgeworfen, in wie weit die Umverteilung der Wirbeligkeit die Strömung beeinflusst und daher die Wirbeligkeit als aktive Substanz behandelt werden muss. In dieser Arbeit soll ein Beitrag zur Beurteilung der Gültigkeit des Modells geliefert werden.

\section{Literaturübersicht}

In einem Übersichtsartikel beschreibt Crighton (1985) die Bedeutung der Kutta-Bedingung für verschiedene Anwendungen. In der Strömungsakustik ist dies z.B. die Erzeugung, Absorption und Streuung von Schall an überströmten Kanten. In den Arbeiten von Howe (1976) zur Schallentstehung an der Hinterkante einer Tragfläche, Bechert (1980) zur Schallabsorption an einem Düsenaustritt, Rienstra (1981) über die Schallstreuung an einer Hinterkante und Ronneberger (1987, 1967/68) zur Schallausbreitung an einem überströmten Querschnittssprung werden unterschiedliche strömungsakustische Aspekte der Annahme einer Kutta-Bedingung untersucht. Auch für aerodynamische Untersuchungen ist die Voraussetzung einer zweckmäßigen Ablösebedingung bedeutend. Satyanarayana \& Davis (1978), Poling \& Telionis (1986), sowie Chen \& Ho (1987) untersuchten den Gültigkeitsbereich der Kutta-Bedingung für ein schwingendes Tragflächenprofil. Basu \& Hancock (1977) berechneten das reibungsfreie Strömungsfeld um ein bewegtes Profil und Türkyilmazoglu et al. (1999) entwikkelten eine numerische Methode zur Simulation des zweidimensionalen Nachlaufs hinter einem Profil. 
Aufgrund ihrer Bedeutung für die instationäre Strömungsablösung finden sich in der Literatur mehrere Beiträge zur Formulierung einer instationären Kutta-Bedingung.

Orszag \& Crow (1970) untersuchten die Auslenkung einer unendlich dünnen Scherschicht unter der Annahme von drei verschiedenen Randbedingungen. Sie prüften eine reine Kutta-Bedingung (full Kutta condition), für die die Scherschichtauslenkung $\eta \sim x^{3 / 2}$ ist. Unter der Annahme der von den Autoren favorisierten gleichgerichteten Kutta-Bedingung (rectified Kutta condition) ist der Winkel, in dem das Fluid von der Platte abströmt, einseitig begrenzt. Aufgrund von Viskositätseffekten sind diese beiden Ablösebedingungen mit instationärem Abschwimmen von Wirbeligkeit von der Platte in die Strömung verbunden. Abschließend wurde auch eine Nicht-Kutta-Bedingung (no Kutta condition) untersucht, bei der die instationäre Wirbeligkeit an der Platte haften bleibt.

Daniels (1978) greift die Überlegungen von Orszag \& Crow (1970) auf und beschreibt ihre Anwendbarkeit für eine viskose Strömung. Er verwendet dazu eine Multi-DeckTheorie, bei der das Gebiet der überströmten Platte in verschiedene Bereiche eingeteilt wird. Diese Abgrenzung richtet sich nach den dominierenden Eigenschaften der Strömung, die in der jeweiligen Region gesondert behandelt werden. Während die gleichgerichtete und die Nicht-Kutta-Bedingung als weniger wahrscheinlich angesehen werden, liefert die Annahme der reinen Kutta-Bedingung konsistente Ergebnisse.

Weitere theoretische Beiträge zur Beschreibung der Auslenkung einer ebenen, unendlich dünnen Scherschicht aufgrund einer äußeren Anregung finden sich noch bei Bechert \& Michel (1975).

Zur Verifizierung und Anwendbarkeit der theoretischen Untersuchungen liefern Experimente einen wichtigen Beitrag. Hierbei ist die Gültigkeit der verschiedenen Annahmen in Abhängigkeit von Strömungs- und Geometrieparametern von besonderem Interesse. In den theoretischen Arbeiten finden sich zu diesem Aspekt nur unzureichende Angaben, da vielfach eine idealisierte Strömung mit einer unendlich dünnen, ebenen Scherschicht untersucht wurde, wie sie in der Realität nicht existiert.

Viele experimentelle Studien beschränken sich bei der Beschreibung der Ergebnisse auf die Abhängigkeit von der Strouhalzahl, also von dem Verhältnis der charakteristischen Reaktionszeit der Strömung zur Periodendauer der Anregung. Eine mögliche 
Abhängigkeit von der Zähigkeit, also von der Reynoldszahl bleibt unberücksichtigt. Obwohl die Resultate auf diese Weise vergleichbar werden, ergibt sich kein einheitliches Bild von der Gültigkeit der quasistationären Kutta-Bedingung.

Für kleine Anregungsfrequenzen kann die Gültigkeit der quasistationären Kutta-Bedingung im Experiment nachgewiesen werden, wie Satyanarayana \& Davis (1978) und Bechert (1980) zeigen. Beim Übergang zu höheren Frequenzen kommt es zunehmend zur Verletzung einer quasistationären Ablösebedingung. Die Untersuchungen u.a. von Satyanarayana \& Davis (1978), Poling \& Telionis (1986), Chen \& Ho (1987) und Bechert \& Stahl (1988) zeigen dies für unterschiedliche Fragestellungen. Die von Ronneberger (1987) experimentell gewonnenen Ergebnisse führen bei höheren Strouhalzahlen zu einer Abweichung von der Theorie, die mit der instationären Verschiebung des Ablösepunktes erklärt werden kann.

Neben der Strouhalzahl ist die Gültigkeit der Theorie auch in Abhängigkeit anderer Parameter zu klären, wie Chen \& Ho (1987) empfehlen. Ein Einfluss der Reynoldszahl wurde z.B. von Bechert \& Pfitzenmaier (1975) bei der Untersuchung eines Düsenstrahls festgestellt. Die Abgrenzungen des Parameterraumes, in dem eine quasistationäre Kutta-Bedingung gültig ist, weichen in sämtlichen der genannten Arbeiten deutlich voneinander $\mathrm{ab}$, so dass weitere Beiträge zur Formulierung eines eindeutigen Kriteriums notwendig sind.

\section{Motivation}

Eine theoretische Formulierung bedarf zum einen einer Überprüfung durch das Experiment und wird zum anderen unter Berücksichtigung sämtlicher relevanter Randbedingungen sehr komplex, so dass die Suche nach einer analytischen Lösung aussichtslos erscheint. Die meisten der genannten theoretischen Abhandlungen beschränken sich auf die Untersuchung einer nichtviskosen Strömung. Die Experimente können deutlich realitätsnäher ausgelegt werden und liefern wertvolle Beiträge besonders zur Anwendbarkeit theoretischer Beschreibungen und Modelle. Sie unterliegen jedoch apparativen Einschränkungen, seien sie technischer oder finanzieller Natur, so dass sich einzelne Aspekte oder z.T. auch eine gesamte Fragestellung der experimentellen Untersuchung entzieht. 
Neben der reinen Theorie und dem reinen Experiment hat sich in letzter Zeit die numerische Simulation als eine dritte Untersuchungsmethode etablieren können. Mit fortschreitender Entwicklung leistungsfähiger Algorithmen und zunehmender verfügbarer Rechenleistung ist auch die Komplexität simulierbarer Prozesse gestiegen.

So geht die Motivation zur Durchführung numerischer Simulationen u.a. aus [Graf (1998)] hervor. Dort wird bemängelt, dass sich der messtechnischen Untersuchung gerade der wichtige Bereich direkt an der Hinterkante aus optischen Gründen entzieht. Mit der dort verwendeten Technik kann man aufgrund optischer Einschränkungen nicht beliebig dicht an der Hinterkante messen, und dies auch nicht mit wünschenswerter Genauigkeit. Gleiches berichten auch [Chen \& Ho (1987)], die zudem noch die Verwendung invasiver Messtechnik ausschließen, aufgrund der Sensitivität der ablösenden Strömung.

Einer Limitierung dieser Art unterliegt die numerische Simulation nicht. Das verwendete Rechengitter kann beliebig fein sein, sofern sich der Bedarf an Ressourcen (Rechenzeit, Speicherbedarf) innerhalb praktikabler Grenzen bewegt. Sämtliche strömungsmechanische Größen wie Geschwindigkeitsvektoren und Druck sind zu jedem Zeitschritt an jedem Gitterpunkt verfügbar. Die Auswertung unterliegt aber auch hier den verfügbaren Kapazitäten.

Darüber hinaus kann im Gegensatz zu den meisten experimentellen Untersuchungen die Antwort der Strömung auf einen Impuls bestimmt werden. Dies stellt zwar hohe Ansprüche an die räumliche und zeitliche Diskretisierung, welche in problemspezifischen Studien ermittelt werden müssen, dafür können aber Hinweise über charakteristische Zeiten direkt erhalten werden. Die Daten gestatten eine Analyse der Dynamik im Zeitbereich, ohne vorherige Transformation des ggf. nicht vollständig abgedeckten Frequenzbereiches.

Es ergeben sich somit großen Chancen der interdisziplinären Untersuchung einer Fragestellung sowohl mit Theorie und Experiment als auch mit numerischer Simulation. Jede Disziplin kann gemäß ihrer Vorzüge einen ergänzenden Beitrag zu einer umfassenden Beschreibung des physikalischen Prozesses liefern. In der vorliegenden Arbeit 
wird dies durch Konzeption, Realisierung und Analyse von Simulationen getan. Diese gehören mit zu den ersten Anwendungen eines neu entwickelten Simulationsprogramms.

\section{Aufbau der Arbeit}

Als Einführung in die Thematik wird im Kapitel 2 „Theorie“ ein kurzer Überblick über den theoretischen Hintergrund vermittelt. Dabei ist die Potenzialströmung und deren Eigenschaften von Bedeutung. Zum einen soll die Anregung in Form eines überlagerten Potenzialfeldes erfolgen. Zum anderen ergibt sich aus der potenzialtheoretischen Beschreibung der Umströmung einer Hinterkante die Notwendigkeit der Annahme einer zusätzlichen Abströmbedingung. Nach der Einführung dieser Kutta-Bedingung wird der Zusammenhang zwischen ihrer Gültigkeit und der Umverteilung von Wirbeligkeit hergestellt. Dazu wird das in [Graf (1998)] vorgestellte „Modell der instationären Strömungsablösung“ erläutert.

Im Kapitel 3 „Numerische Simulation“ werden zunächst die wichtigsten Merkmale des verwendeten $\tau^{\mathrm{i}}$-Codes beschrieben. Da diese eng mit der Art der Realisierung der zugrunde liegenden Fragestellung verknüpft sind, widmen sich weitere Abschnitte speziellen Studien der Umsetzbarkeit des zu untersuchenden Strömungsszenarios. Es werden u.a. Anforderungen an die Diskretisierung ermittelt, um die Zuverlässigkeit und Genauigkeit der Rechnungen zu gewährleisten.

Unter Beachtung der in Kapitel 3 ermittelten Voraussetzungen zur Durchführung einer verlässlichen Simulation werden in Kapitel 4 die Ergebnisse der getrennten Berechnung der stationären Gleichströmung und des überlagerten Strömungsfeldes vorgestellt. Dabei kommt der Entwicklung der Einströmrandbedingungen besondere Bedeutung zu, da diese für die Eigenschaften der resultierenden Strömungsfelder maßgeblich sind.

Die Ergebnisse der Simulation der Reaktion der Gleichströmung auf einen Druckgradientenimpuls werden in den nachfolgenden Kapiteln dargestellt. Im Kapitel 5 wird die Dynamik der Auslenkung der Scherschicht in Raum und Zeit untersucht. Grundlage hierzu ist das 1te Moment der Wirbeligkeitsverteilung. Dessen zeitlicher Verlauf deutet zwar auf eine Besonderheit der instationären Strömungsablösung hin, reicht jedoch zur vollständigen Beschreibung des Vorganges nicht aus. Im folgenden Kapitel 6 „Wech- 
selwirbeligkeit“ erfolgt daher die Betrachtung des Feldes des durch die Anregung Entstandenen Anteils der Wirbeligkeit. Dessen zeitliche Entwicklung beschreibt den Relaxationsprozess anhand der im Modell verwendeten Größe der Wirbeligkeit.

Nachfolgend werden die Ergebnisse der numerischen Simulationen in Kapitel 7 zusammengefasst. Hieraus lassen sich Schlussfolgerungen zum Zusammenhang der instationären Kutta-Bedingung mit der Umverteilung von Wirbeligkeit ziehen. Aus diesen leiten sich Anregungen für weiterführende numerische Untersuchungen ab. 


\section{Theorie}

In diesem Kapitel sind einige grundlegende Betrachtungsweisen der instationären Strömungsablösung zusammengefasst. Der in Abschnitt 2.1 beschriebenen Potenzialströmung kommt doppelte Bedeutung zu. Zum einen weist die plötzliche Änderung des Strömungsfeldes Eigenschaften einer Potenzialströmung auf; dadurch vereinfacht sich die Betrachtung des Ablöseprozesses anhand der Wirbelverteilung. Zum anderen erwächst aus der potenzialtheoretischen Beschreibung der Strömung an einer Kante die Notwendigkeit der Annahme einer zusätzlichen Ablösebedingung. Diese sog. KuttaBedingung wird im Abschnitt 2.2 vorgestellt. Die Übertragung der Kutta-Bedingung auf das Ablösen einer instationären Strömung konnte bisher noch nicht vollständig erfolgen. Grund dafür ist die Komplexität der Interaktion elementarer Einzelprozesse. Ein Modell, welches in Abschnitt 2.3 erläutert wird, soll dieses Strömungsszenario einer Interpretation zuführen.

\subsection{Potenzialströmung um eine Kante}

Wird eine Strömung nach der Potenzialtheorie beschrieben, so wird ein homogenes, reibungsfreies Fluid betrachtet, dessen Bewegung überall im Innern des Strömungsgebietes wirbelfrei ist. Für inkompressible Fluide stellen Potenzialströmungen unter geeigneten Randbedingungen exakte Lösungen der Navier-Stokes-Gleichung dar. Die geforderte Wirbelfreiheit gestattet nur die Verwendung schubspannungsfreier Randbedingungen, da im Falle einer Haftrandbedingung Wirbeligkeit in das Strömungsfeld diffundiert und dieses damit nicht länger rotationsfrei ist. Somit ist allgemein nur die Beschreibung eines reibungsfreien Fluids möglich.

Eine Übertragung der Ergebnisse einer reibungsfreien Berechnung auf eine viskose Strömung sind nur dann sinnvoll, wenn die Strömung nicht ablöst. Die Simulation einer Tragflügelumströmung z.B. nach der vortex-panel-Methode, bei der das Geschwindigkeitsfeld aus der einzelnen Oberflächenelementen zugeordneten Wirbelstärke berechnet wird, liefert viele gleichrangige Lösungen. Erst mit der Annahme einer zusätzlichen Abströmbedingung läßt sich das korrekte Ergebnis ermitteln [Anderson in VKI (1999)]. 
Nach der Methode der konformen Abbildung oder mit der Schwarz-Christoffel-Transformation (z.B. aus [Spurk (1996)]) läßt sich die Umströmung eines Körpers gemäß der Potenzialtheorie berechnen. In Abbildung 2.1 ist dies für die Umströmung einer Hinterkante dargestellt.

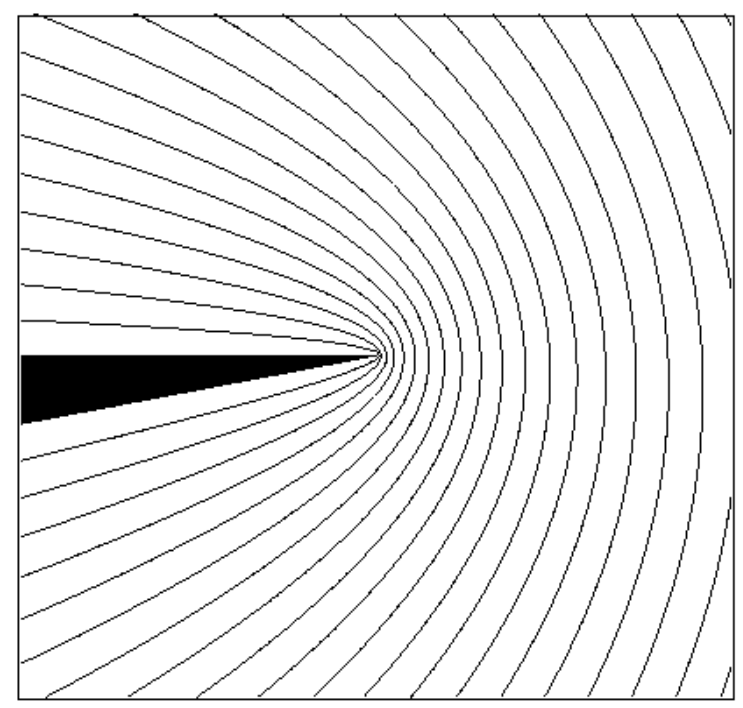

Abbildung 2.1: Stromlinien der Umströmung einer Hinterkante (Abbildung aus [Graf (1998)], berechnet nach der Potenzialtheorie)

Die Potenzialströmung verläuft wirbelfrei. Ist zur Umströmung einer Oberfläche eine Richtungsänderung notwendig, so ist damit auch eine Kraft, bzw. ein Druckgradient verbunden. Für eine Hinterkante entspricht dies der Zentripetalkraft, welche auch vom Radius der Kante abhängt. Geht dieser Radius gegen Null, so ist eine unendliche Kraft, hier ein Unterdruck, notwendig. Nach der Gleichung von Bernoulli erfordert dies eine unendlich schnelle Umströmung der Kante!

\subsection{Kutta-Bedingung}

Erst die Annahme einer Ablösebedingung zusätzlich zur potenzialtheoretische Umströmung der Hinterkante führt zu einem realistischen Strömungsfeld. In Abschnitt 2.2.1 werden einige gleichwertige Formulierungen dieser Bedingung aufgeführt und deren Entdeckung kurz skizziert. Ihre Gültigkeit ist für das Ablösen einer stationären Strömung unumstritten. Im instationären Fall jedoch sind die beteiligten Prozesse und ihre Wechselwirkungen deutlich komplexer, so dass eine ähnlich allgemeingültige Ablösebedingung noch nicht formuliert werden konnte, wie in Abschnitt 2.2.2 erläutert ist. 


\subsubsection{Definition und Historie}

Nach der Potenzialtheorie ergibt sich für die nicht viskose Umströmung einer Hinterkante eine Singularität der Strömungsgeschwindigkeit an der Kante. Dort ist die Geschwindigkeit unbegrenzt, und somit auch die auf die Kante wirkende Kraft. Aufgrund der für eine laminare, viskose Strömung geltenden Haftbedingung, muss die Geschwindigkeit des Fluids dort aber gleich null sein. Dieser Gegensatz ist aufgehoben, wenn zusätzlich eine Ablösebedingung angenommen wird, so dass die Singularität an der Kante möglichst gering ist. Für eine stationäre Strömung kann die Bedingung alternativ formuliert werden (z.B. [Poling \& Telionis (1986)]):

1) Die Druckverteilung um die Kante herum ist kontinuierlich

2) Die hintere Staupunkt-Stromlinie beginnt in der Hinterkante und teilt ihren Winkel

3) Die Umströmungsgeschwindigkeit ist begrenzt oder null.

Die Kutta-Bedingung, eigentlich Kutta-Joukowski-Hypothese, wurde von Kutta (1902) und Joukowski (1904) unabhängig voneinander bei der Untersuchung der Tragflügelumströmung gefunden. Bei der theoretischen Berechnung der Auftriebskraft eines Flügels fanden sie, dass diese genau proportional eines zirkulatorischen Anteils ist. Erst dessen Annahme führt zu einer korrekten Umströmung, wie Abbildung 2.2 zeigt. Das resultierende Strömungsfeld genügt auch den zuvor genannten gleichwertigen Formulierungen der Kutta-Bedingung.
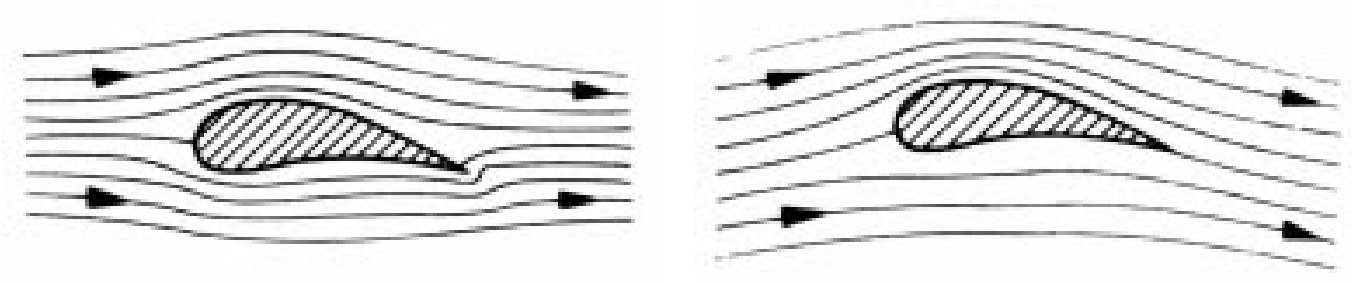

Abbildung 2.2: Stromlinien einer einfachen Potenzialströmung um einen Flügel ohne zirkulatorischen Anteil (links) im Vergleich zu einer realen Flügelumströmung mit Zirkulation (rechts)

(Abbildungen aus [Prandtl (1990)]) 
Im Folgenden wird die instationäre Strömungsablösung hauptsächlich durch die Umverteilung von Wirbeligkeit beschrieben. Die über der Platte entstehende Grenzschicht schwimmt ab und geht an der Hinterkante in eine freie Scherschicht über. Für eine stationäre Strömung läßt sich eine adäquate Kutta-Bedingung so definieren:

Die Wirbeligkeitsschicht löst tangential von der Hinterkante ab.

\subsubsection{Gültigkeit für instationäre Strömungen}

Wird eine stationär ablösende Scherschicht durch eine Störung ausgelenkt, so kann i.a. nicht vorausgesetzt werden, dass die klassische Kutta-Bedingung weiterhin uneingeschränkt gültig ist. Ist die Periodendauer der Anregung groß gegenüber der charakteristischen Antwortzeit, bzw. Relaxationszeit, der Strömung, so ist ein im Wesentlichen tangentiales Ablösen zu beobachten. Dies entspricht der Gültigkeit einer quasistationären Kutta-Bedingung. Für schnelle Änderungen der Anregung ist diese Annahme nicht mehr zulässig, wie verschiedene Untersuchungen zeigen (s. Literaturübersicht). Verschiedene Prozesse treten in diesem Fall in Wechselwirkung. Eine eindeutige Identifikation der beteiligten charakteristischen Mechanismen, bzw. deren quantitative Beschreibung, war bisher noch nicht möglich. Dies ist jedoch die Voraussetzung für die Formulierung einer allgemein gültigen Kutta-Bedingung für das Ablösen einer instationären Strömung.

\subsection{Modell der instationären Strömungsablösung}

Ein Modell soll die Wechselwirkungen zwischen einer instationären Strömung und einer einlaufenden Störung durch das Zusammenwirken elementarer Einzelprozesse beschreiben und so die maßgeblichen Eigenschaften von Strömung und Anregung einer Interpretation zuführen. Im Abschnitt 2.3.1 wird die Strömungsablösung durch ein Modell des Wirbeltransports beschrieben. Die charakteristischen Zeiten zur Auslenkung und Relaxation der Strömung werden in Abschnitt 2.3.2 aus den grundlegenden physikalischen Prozessen der Diffusion und Konvektion von Wirbeligkeit abgeleitet. Es zeigt sich, dass diese Zeiten und damit die gesamte Dynamik der Strömungsablösung sowohl von der Strouhal- als auch von der Reynoldszahl abhängen sollten. Dabei deutet sich allerdings schon an, dass die Reynoldszahl eine geringere Rolle als die Strouhalzahl spielt, einerseits, weil die Strouhalzahl sehr viel stärker variieren kann als die Reynoldszahl (bei hohen Reynoldszahlen ist die laminare Wand- 
grenzschicht instabil; kleine Reynoldszahlen kommen bei ablösenden Strömungen praktisch nicht vor), andererseits, weil die Reynoldszahl mit einer kleineren Potenz eingeht als die Strouhalzahl. Beide Kennzahlen ermöglichen den Vergleich unterschiedlicher Strömungen hinsichtlich der Gültigkeit der quasistationären Kutta-Bedingung. In Abschnitt 2.3.3 wird die qualitative Abhängigkeit der Ablösebedingung von der Strouhalzahl erläutert.

\subsubsection{Modellvorstellung}

In einem heuristischen Modell wird die Dynamik der Strömungsablösung von der Hinterkante durch den Transport von Wirbeligkeit betrachtet, wie sie in [Graf (1998)] beschrieben ist. Das stationäre Feld der Gleichströmung, bei der die Kutta-Bedingung erfüllt ist, hat eine entsprechende stationäre Wirbeligkeitsverteilung. Diese ist näherungsweise unabhängig von der Koordinate in Hauptströmungsrichtung (x-Koordinate). Die Verteilung wird durch eine Anregung gestört, die mit der Erzeugung zusätzlicher Wirbeligkeit verbunden ist. Durch Umverteilung innerhalb einer Relaxationszeit stellt sich eine neue, stationäre Wirbeligkeitsverteilung ein.

Die Anregung der Gleichströmung durch einen Druckgradienten-Impuls zur Zeit $\mathrm{t}=0$ führt zur Überlagerung einer Potenzialströmung um die Kante herum, wie in Abbildung 2.3 dargestellt ist. Die somit erfolgte Verletzung der klassischen Ablösebedingung ist gleichzeitig auch mit der Produktion von Wirbeligkeit an der Oberfläche der Splitterplatte verbunden. Wirbeligkeit kann in der zweidimensionalen Strömung ausschließlich an einer festen Oberfläche erzeugt werden. Unmittelbar nach der Anregung gleitet die Strömung an der Wand entlang, d.h. dort entsteht eine theoretisch unendlich hohe Wirbeligkeit, die somit eine Deltaverteilung aufweist. An der Hinterkante findet man eine doppelte Singularität. Zum einen ist auch dort die Wirbeligkeitsschicht unendlich dünn, zum anderen wird die Kante mit unendlich hoher Geschwindigkeit umströmt. Die durch die Überlagerung einer Potenzialströmung neu entstandenen Wirbeligkeit konzentriert sich daher vornehmlich auf den Bereich der Hinterkante.

Da das Fluid nahe der Wand aufgrund der Haftbedingung nahezu ruht, verbreitert sich die Grenzschicht zunächst hauptsächlich durch Diffusion. Für wirbeligkeitsbehaftete Fluidelemente weiter entfernt von der Wand in der Strömung ist dagegen die Konvek- 


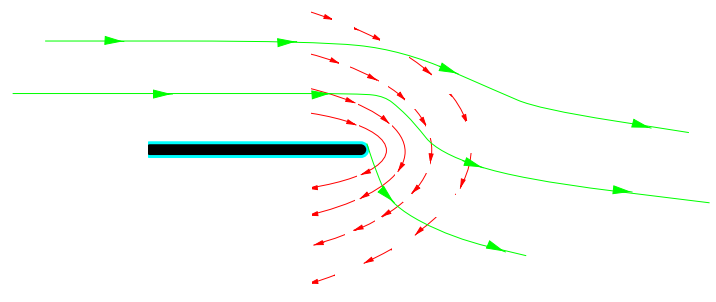

Abbildung 2.3: Anregung durch ein Potenzialfeld: Der Strömung wird ein Druckgradient aufgeprägt, dies führt zu einer plötzlichen Erhöhung der Strömungsgeschwindigkeit und zu einer Umströmung der Hinterkante. (Abbildung aus [Graf (1998)])

tion maßgeblich. Sie schwimmen innerhalb der sog. „Transitzeit“ durch das Nahfeld der Kante. Diese Fluidelemente werden entlang der durch das überlagerte Potenzialfeld ausgelenkten Stromlinien der Gleichströmung transportiert. Ihre Wirbeligkeit ändert sich nur wenig, wenn die Konvektionszeit kurz ist im Vergleich zur Diffusionszeit.

Die Verschiebung der Fluidelemente äußert sich in einer zeitabhängigen Auslenkung der Scherschicht, wie Abbildung 2.4 zeigt. Die somit erfolgte Verletzung der klassischen Ablösebedingung ist daher eng mit dem Versatz der Wirbeligkeitsverteilung der Gleichströmung und gleichzeitig auch mit der Produktion von Wirbeligkeit an der Oberfläche, bzw. Hinterkante der Splitterplatte verbunden.

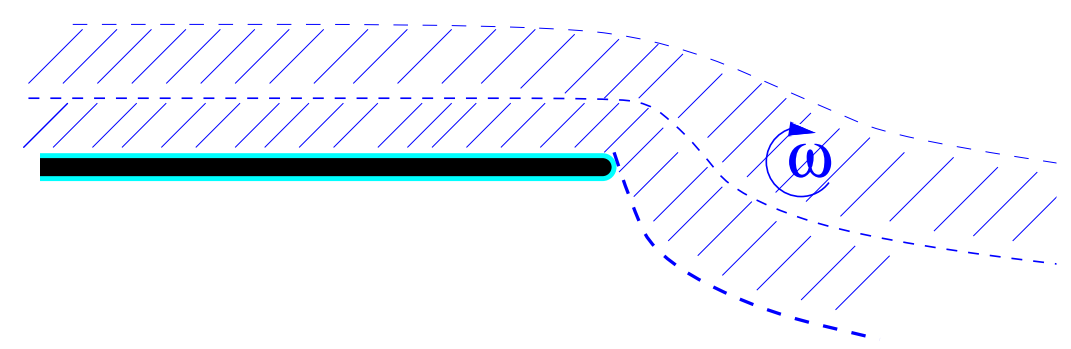

Abbildung 2.4: Ausgelenkte Wirbeligkeitsschicht: Wirbeligkeitsbehaftete Fluidteilchen folgen den veränderten Stromlinien, was zu einer Auslenkung der Wirbeligkeitsschicht führt.

(Abbildung aus [Graf (1998)])

Erst nach der Umverteilung der Wirbeligkeit durch Konvektion und Diffusion innerhalb einer „Relaxationszeit“, geht die Strömung in einen neuen, stationären Endzustand über. Während diese Prozesses kann es aufgrund einer möglichen Rückwirkung der umverteilten Wirbeligkeit auf die Strömung auch zur Produktion von entgegengerichteter Wirbeligkeit und damit verbunden auch zu einer Kompensation kommen. Die 
durch die Anregung erzeugte Wirbeligkeit hat sich auf die gesamte Scherschicht verteilt. Diese enthält ebenso wie die einlaufende Grenzschicht aufgrund der erhöhten Strömungsgeschwindigkeit mehr Wirbeligkeit als im Ausgangszustand, wie in Abbildung 2.5 dargestellt ist. Die Umverteilung der Wirbeligkeit kennzeichnet den Abbau der Kantenumströmung und somit auch der Scherschichtauslenkung. In diesem relaxierten Zustand gilt nun wieder die Kutta-Bedingung und die Strömung verhält sich somit quasistationär. Die Gültigkeit dieser quasistationären Kutta-Bedingung ist dort verletzt, wo die Änderungen der Strömungsgeschwindigkeit schnell sind, im Vergleich zur benötigten Relaxationszeit (u.a. [Poling \& Telionis (1986)]).

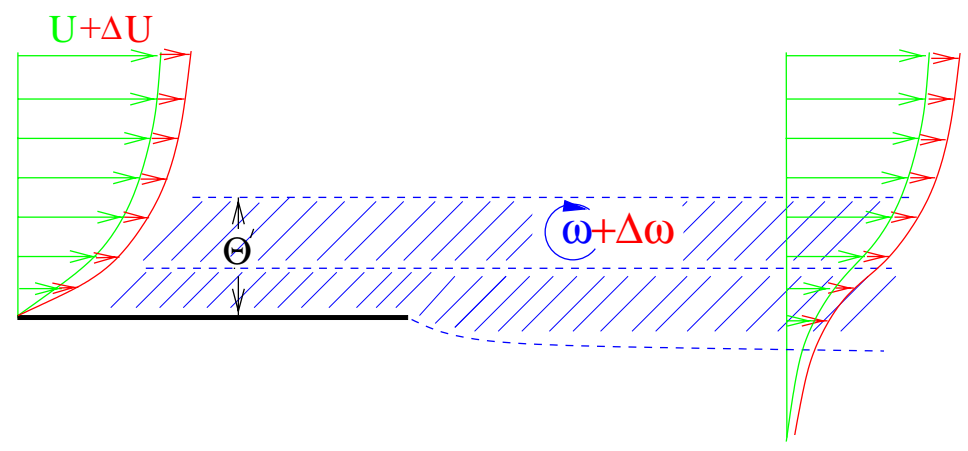

Abbildung 2.5: Stationärer Endzustand: Durch die Umverteilung von Wirbeligkeit relaxiert die Strömung in einen neuen Zustand. Die an der Plattenoberfläche erzeugte Wirbeligkeit wird durch Konvektion und Diffusion mit der Strömung bzw. in die Strömung hinein transportiert. (Abbildung aus [Graf (1998)])

\subsubsection{Zeitskalen des Modells}

Die Beschreibung der Dynamik der Strömungsablösung von der Hinterkante durch den Transport von Wirbeligkeit nach dem heuristischen Modell ist eng mit den charakteristischen Zeiten der Gleichströmung verbunden. Dem Modell gemäß werden die Zeitskalen aus den einzelnen, grundlegenden Strömungsvorgängen der Konvektion und der Diffusion abgeleitet. 
Die Konvektion wird von der Gleichströmung dominiert. Die Konvektionszeit $\mathrm{T}_{\text {konv }}$ wird daher definiert, als die Zeit, in der ein Fluidelement in der Hauptströmung eine Strecke der Größe der Impulsverlustdicke $\Theta$ zurücklegt:

$$
\mathrm{T}_{\text {konv }} \sim \frac{\Theta}{\mathrm{U}_{\infty}}
$$

In der Diffusionszeit $\mathrm{T}_{\text {diff }}$ diffundiert ein wirbeligkeitsbehaftetes Fluidelement entsprechend über eine Strecke der Größe der Impulsverlustdicke $\Theta$ :

$$
\mathrm{T}_{\mathrm{diff}} \sim \frac{\Theta^{2}}{\mathrm{v}}
$$

mit $v$ : kinematische Zähigkeit. Diese Beziehung leitet sich aus der Lösung der eindimensionalen Diffusionsgleichung (Navier-Stokes-Gleichung für den ebenen Fall) ab:

$$
\frac{\partial u(y, t)}{\partial t}=v \frac{\partial^{2} u(y, t)}{\partial y^{2}}
$$

Es wurden dazu die Randbedingungen eines ruhenden Fluids über einer plötzlich in Bewegung gesetzten Platte verwendet. Diese finden auch im Abschnitt 4.2.1, „Instationäre Einströmrandbedingung“ auf Seite 46, bei der numerischen Nachbildung der Diffusion von der Platte am Einströmrand Anwendung. Sie sind dort näher erläutert.

Aus der Beschreibung der einzelnen Phasen der instationären Scherschichtauslenkung nach der Wirkungsweise von Diffusion und Konvektion können die zugehörigen Zeiten der Anregung und Relaxation der Strömung abgeschätzt werden.

Nach einer impulsartigen Störung zur Zeit $t=0$ wird die Scherschicht ausgelenkt. Dies geschieht innerhalb der Transitzeit $\mathrm{T}_{\text {transit }}$, in der ein Fluidelement durch das Nahfeld der Kante strömt. Als Nahfeld wird der Bereich um die Kante bezeichnet, in dem sich das angeregte Potenzialfeld mit dem Gleichströmungsfeld überlagert. Die Transitzeit ist ausschließlich von der Konvektionszeit abhängig. Als charakteristische Länge, d.h. als Strecke, die das Fluidelement innerhalb der Transitzeit zurücklegt, wird auch hier die Impulsverlustdicke $\Theta$ verwendet. 
Nach einer Relaxationszeit $T_{\text {relax }}$ nimmt die Strömung wieder einen stationären Zustand ein. Sie wird definiert als die Zeit, innerhalb derer die Spitze der Wirbeligkeitsverteilung über der Platte eine Strecke der Länge der Impulsverlustdicke zurückgelegt hat. Unter Berücksichtigung von Diffusion und Konvektion ist die Abschätzung der Relaxationszeit nach [Graf (1998)]:

$$
\mathrm{T}_{\text {relax }} \sim\left(\frac{\Theta}{\mathrm{U}_{\infty}}\right)^{\frac{2}{3}} \cdot\left(\frac{\Theta^{2}}{\mathrm{v}}\right)^{\frac{1}{3}}=\left(\mathrm{T}_{\mathrm{konv}}\right)^{\frac{2}{3}} \cdot\left(\mathrm{T}_{\mathrm{diff}}\right)^{\frac{1}{3}}
$$

Die Konvektion ist der dominierende Prozess, sowohl beim Transit der Teilchen durch das Nahfeld der Hinterkante, als auch bei der Relaxation der Strömung in den stationären Zustand. Aus der zugehörigen Konvektionszeit kann ein Maß für die Gültigkeit der quasistationären Kutta-Bedingung abgeleitet werden. Es ist zu erwarten, dass die Bedingung dann erfüllt ist, wenn die Relaxation abgeschlossen ist, d.h wenn die Wirbeligkeit umverteilt wurde. Das Verhältnis aus Periodendauer T der Anregung und der Konvektionszeit der Strömung beschreibt die zeitliche Relation zwischen der Produktion und der Umverteilung von Wirbeligkeit. Es ist daher auch ein Maß für die Gültigkeit der quasistationären Kutta-Bedingung. Dieses Verhältnis entspricht bis auf den Faktor $2 \pi$ der Strouhalzahl, gebildet mit der Impulsverlustdicke:

$$
\mathrm{St}_{\Theta}=\frac{2 \pi \mathrm{fD}}{\mathrm{U}_{\infty}}=\frac{2 \pi \Theta}{\mathrm{TU}_{\infty}}=2 \pi \frac{\mathrm{T}_{\mathrm{konv}}}{\mathrm{T}}
$$

$\mathrm{f}$ ist die Frequenz der Anregung, mit $\mathrm{f}=\frac{1}{\mathrm{~T}}$ und $\mathrm{D}$ die typische Länge, hier die Impulsverlustdicke $\Theta$.

Die Simulationen im Rahmen dieser Arbeit wurden für Gleichströmungen mit verschiedener Viskosität $v$ durchgeführt. Diese Größe beeinflusst die Diffusion entscheidend, bleibt jedoch bei der Charakterisierung der instationären Ablösung ausschließlich durch die Strouhalzahl unberücksichtigt. Als weiteres Kriterium für die Gültigkeit der Kutta-Bedingung ist daher das Verhältnis aus Diffusionszeit zu Konvektionszeit geeignet. Es entspricht der mit der Impulsverlustdicke gebildeten Reynoldszahl:

$$
\operatorname{Re}_{\Theta}=\frac{\mathrm{T}_{\text {diff }}}{\mathrm{T}_{\text {konv }}}=\frac{\mathrm{U}_{\infty} \Theta}{\mathrm{v}}
$$




\subsubsection{Ablösebedingungen}

In einigen theoretischen Arbeiten (z.B. [Orszag \& Crow (1970)] und [Daniels (1978)]) wurden verschiedene Formen der Scherschichtauslenkung unter der Annahme einer entsprechenden Ablösebedingung diskutiert. Deren Gültigkeit in Abhängigkeit von der Strouhalzahl wurde in Experimenten (z.B. [Satyanarayana \& Davis (1978)] und [Poling \& Telionis (1986)]) ermittelt. Im Folgenden werden die möglichen Ablösebedingungen verschiedenen Phasen der oben beschriebenen Wirbelumverteilung zugeordnet. Damit ergeben sich bestimmte Intervalle der Strouhalzahl, in denen die Bedingungen gültig sein sollten (s. Abbildung 2.6):

a.)

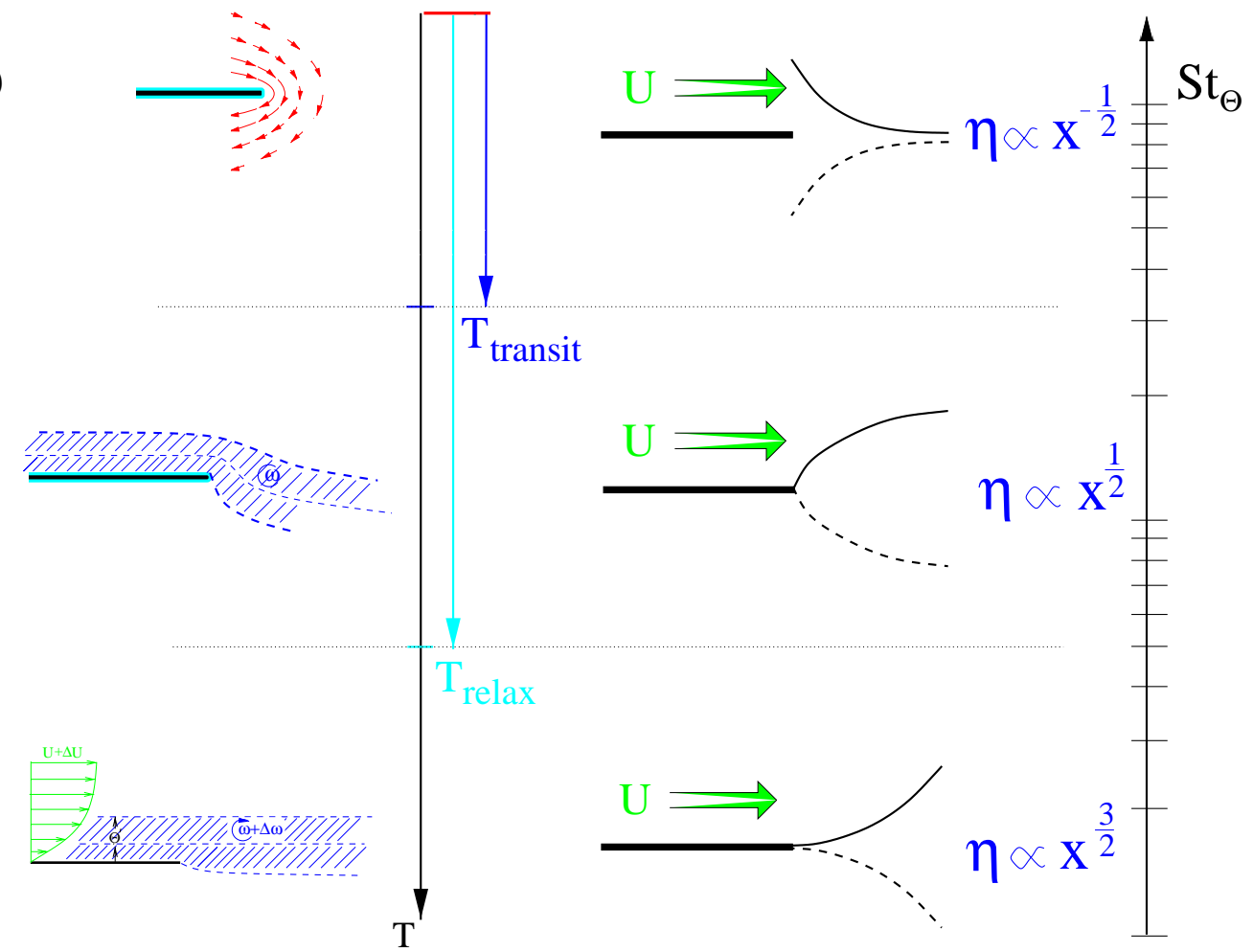

b.)

Abbildung 2.6: Phasen und Formen der Scherschichtauslenkung und deren Zusammenhang mit den charakteristischen Zeiten, die sich aus dem Modelle ableiten lassen.

(Abbildung aus [Graf (1998)]) 
Es lassen sich folgende Fälle unterscheiden:

a) Für hohe Frequenzen $\mathrm{St}_{\Theta}$ » 1 , bei denen die Periodendauer der Anregung sehr viel kürzer ist, als die Transitzeit, ist die Beschleunigung der Teilchen durch die Anregung deutlich größer, als durch die Strömung. Während einer Periode ändern sie ihren Ort x nur unwesentlich, daher ist die Auslenkung $\eta$ der Wirbeligkeitsschicht von der Geschwindigkeit des Potenzialfeldes abhängig. Diese ist proportional $\mathrm{x}^{-1 / 2}$, also:

$$
\eta(x) \sim x^{-1 / 2} \text { für } \mathrm{St}_{\Theta} \gg 1
$$

b) Die Teilchen folgen den ausgelenkten Stromlinien der Gleichströmung durch das Nahfeld der Kante, wenn die Periodendauer der Anregung groß gegen die Transitzeit und klein gegen die Relaxationszeit ist. Es gilt:

$$
\eta(\mathrm{x}) \sim \mathrm{x}^{1 / 2} \text { für } 1 \gg \mathrm{St}_{\Theta} \gg \frac{\mathrm{T}_{\text {transit }}}{\mathrm{T}_{\text {relax }}}
$$

c) Die quasistationäre Kutta-Bedingung ist dann gültig, wenn die Periodendauer der Anregung wesentlich größer als die Relaxationszeit der Strömung ist:

$$
\eta(x) \sim x^{3 / 2} \text { für } \frac{T_{\text {transit }}}{T_{\text {relax }}} \gg S t_{\Theta}
$$

Die Darstellung der Strömungsablösung (Abbildung 2.6) in diskreten, einzelnen Ablöseformen findet man allenfalls für hohe Reynoldszahlen. Allgemein muss davon ausgegangen werden, dass die Prozesse nicht unabhängig voneinander und nacheinander, sondern überlappend stattfinden. Die Überlappung wird mit abnehmender Reynoldszahl zunehmen. 


\section{Numerische Simulation}

In diesem Kapitel werden die numerischen Grundlagen vorgestellt, denn die Möglichkeiten und Limitierungen einer numerischen Simulation sind eng mit den Merkmalen des verwendeten Strömungslösers verbunden. Es bestehen fundamentale Unterschiede z.B. bei der Berechnung einer kompressiblen und einer inkompressiblen Strömung. Ein Code für kompressible Strömungen bedarf daher zur Simulation inkompressibler Strömungen einer Vorkonditionierung, dem gegenüber ist aber die Verwendung eines Codes ausschließlich zur Berechnung inkompressibler Strömungen vorzuziehen. Bei der Abbildung des Simulationsgebietes auf ein Rechengitter unterliegt die Wahl der elementaren Zelltypen (z.B. Prismen, Hexaeder) den Verarbeitungsmöglichkeiten des Strömungslösers. Nicht zuletzt ist Anwendbarkeit und die resultierende Genauigkeit des Simulationsergebnisses stark von den implementierten Algorithmen abhängig. Die Wahl des Werkzeugs ist somit eng mit Aufgabenstellung verknüpft.

In Abschnitt 3.1 wird ein Überblick über das verwendete Software-Paket, den DLR TAU-Code, gegeben. Eine Besonderheit bietet die Vorverarbeitung (Preprocessing) der Gitterdaten, sie ist in Abschnitt 3.1.1 beschrieben. Daran schließt sich in Abschnitt 3.1.2 die Beschreibung des Strömungslösers für inkompressible Fluide an. Da das Programm in einer ersten Version vorlag und sein Verhalten bei verschiedenartigen Strömungen noch nicht vollständig bekannt war, wurde eine Validierung nach der DFG-Benchmarkstudie durchgeführt. Diese ist im Anhang, Abschnitt A.2 auf Seite 89, dokumentiert. Damit konnte die Genauigkeit der Lösung abgeschätzt werden. Die Generierung des Rechengitters ist der erste grundlegende Schritt zur Simulation der instationären Scherschicht. In Abschnitt 3.2 ist dieser Prozess und die problemspezifischen Anforderungen daran näher erläutert. Den Bedingungen am Rand des Simulationsgebietes kommt in doppelter Hinsicht besondere Bedeutung zu. Zum einen wird die Strömung berechnet durch Lösen eines Anfangsrandwertproblems, zum anderen stellt der Rand die Grenze der räumlichen Diskretisierung dar, d.h. Randpunkte haben nicht wie Feldpunkte Nachbarelemente in jeder Richtung. Dies macht eine besondere Behandlung der festen Ränder bei der Berechnung der Wirbeligkeit notwendig (Abschnitt 3.3). Wie bereits erwähnt beeinflussen sowohl die Eigenschaften des 
Lösers, als auch die der zu simulierenden Strömung die Verhaltensweise des Lösungsprozesses und die Charakteristik des Ergebnisses. In Abschnitt 3.4 ist dies für die instationäre Simulation der Scherschicht dargestellt.

\subsection{DLR TAU-Code}

Die numerischen Simulationen wurden mit dem DLR TAU-Code [Schwamborn, Gerhold, Kessler (1999)] durchgeführt. Auf die verwendeten Module dieser Software wird in den folgenden Abschnitten näher eingegangen. Aus dem bereitgestellten primären Gitter generiert das Preprocessing-Modul das duale Gitter (Abschnitt 3.1.1), und die zugehörigen Mehrgitter-Ebenen, auf welchen der Löser dann arbeitet. Der TAU-Code beinhaltet einen Gleichungslöser zur Simulation kompressibler Strömung und einen zur Berechnung inkompressibler Strömung ( $\tau^{\mathrm{i}}$-Code), dieser wird hier ausschließlich verwendet und in Abschnitt 3.1.2 beschrieben.

\subsubsection{Preprocessing}

Eine Aufgabe des Preprocessing-Moduls ist die Konstruktion des eigentlichen Gitters, auf dem der Strömungslöser arbeitet. Dazu wird aus dem vorgegebenen primären Gitter, welches Zellen mit sehr ungünstigem Seitenverhältnis beinhalten kann, ein sog. duales Gitter generiert, welches aus sehr kompakten, konvexen Elementen besteht, deren Struktur zur Erhöhung der Genauigkeit des Simulationsergebnisses beiträgt. In Abbildung 3.1 ist ein Ausschnitt des primären Gitters (breitere Linien) und das zugehörige duale Gitter dargestellt.

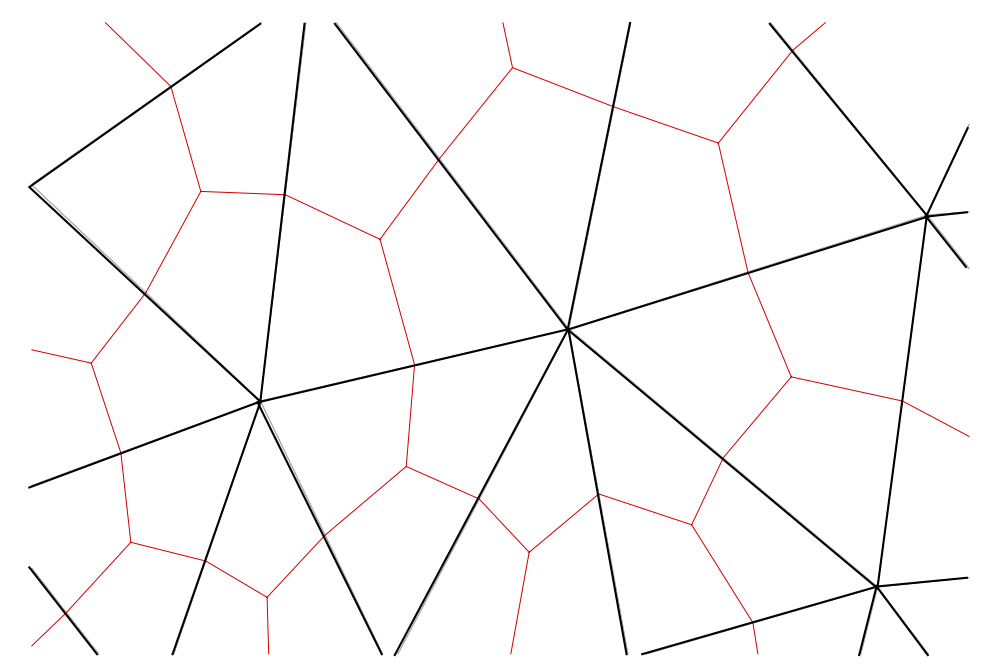

Abbildung 3.1: Primäres und duales Gitter 
Des weiteren werden bei dieser Vorverarbeitung alle geometrischen und logischen Daten berechnet und in einer kantenbasierten Datenstruktur gespeichert, d.h. alle Informationen werden einer Verbindungslinie zwischen zwei benachbarten Elementen, also einer Kante, zugeordnet. Auf diese Weise kann der Speicherbedarf reduziert und die Flexibilität der Daten gesteigert werden. Das ursprüngliche primäre Gitter ist für die eigentliche Simulation nun überflüssig und steht dem Löser nicht zur Verfügung.

Zur effizienten Lösung der Druckgleichung ist im Gleichungslöser ein Mehrgitteralgorithmus zur Konvergenzbeschleunigung implementiert. Die dazu notwendigen gröberen Gitter werden ebenfalls im Preprocessing aus dem vorhandenen feinsten dualen Gitter durch Agglomeration von Kontrollvolumina erzeugt. Dazu werden die Zellen eines feinen Gitters zu Elementen des nächstgröberen Gitters verschmolzen. Die Robustheit und Effizienz des Verfahrens wird dadurch gewährleistet, dass auch lokal strukturierte Bereiche, wenn auch nicht so gut aufgelöst, erhalten bleiben, wie z.B. die Hinterkante bei dem in Abbildung 3.2 dargestellten Agglomerationsprozess. Bei unstrukturierten Gittern ist die Mehrgittermethode noch Gegenstand aktueller Forschung, nicht so bei strukturierten Gittern, wo sie zum Standard gehört.

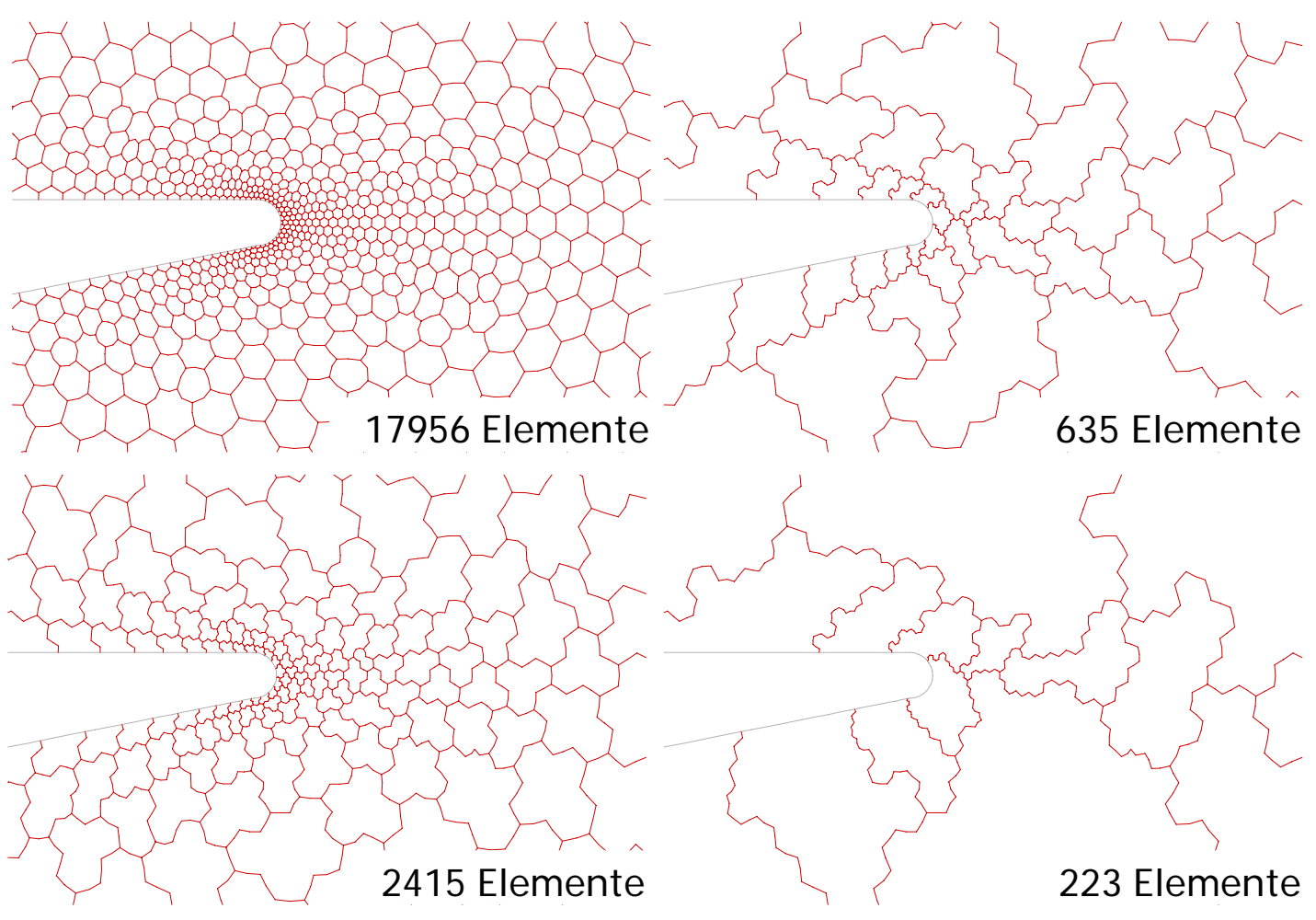

Abbildung 3.2: Schnitt durch die Symmetrie-Ebene dualer Zellen aufeinander folgender vergröberter Gitter.

Zum Vergleich: Hinterkante $1.5 * 10^{-4} \mathrm{~m}$ dick 


\subsubsection{Gleichungslöser}

Aus den vorgegebenen Anfangsrandwerten berechnet der Gleichungslöser das resultierende viskose, instationäre, laminare, inkompressible Strömungsfeld, bestehend aus dem Geschwindigkeitsvektor und einem Druckwert, durch Lösen der Navier-StokesGleichungen. Diese Grundgleichungen der Strömungsmechanik bestehen aus einer Impuls- (3.1) und einer Kontinuitätsgleichung (3.2), die für jedes Element des Rechengitters gelöst werden.

$$
\begin{gathered}
\frac{\partial}{\partial \mathrm{t}} \overrightarrow{\mathrm{u}}+(\overrightarrow{\mathrm{u}} \cdot \operatorname{grad}) \overrightarrow{\mathrm{u}}+\frac{1}{\rho} \operatorname{gradp}=v \Delta \overrightarrow{\mathrm{u}} \\
\operatorname{div} \overrightarrow{\mathrm{u}}=0
\end{gathered}
$$

Der Löser arbeitet nach dem Finite-Volumen-Verfahren auf unstrukturierten, hybriden Gittern. Alle Variablen werden nicht-versetzt (colocated) an jeweils einer Position im Gitterelement berechnet und gespeichert.

Bei der Berechnung von inkompressiblen Strömungen ist die Kopplung von Druckund Geschwindigkeitsfeldern besonders entscheidend. Bei kleinen Machzahlen sind Druck und Dichte nahezu entkoppelt, daher arbeiten Verfahren zur Simulation kompressibler Strömungen hier wenn überhaupt nur ineffektiv. $\operatorname{Im} \tau^{\mathrm{i}}$-Code wurde ein Projektionsverfahren implementiert, wobei die Impulsgleichungen und die Druckberechnung in jedem Zeitschritt nicht direkt gekoppelt sind. Gelöst werden zunächst die Impulsgleichungen mit dem Druck des letzten Zeitschrittes. Die hieraus resultierende Geschwindigkeitsverteilung erfüllt die Kontinuitätsgleichung erst dann wieder, wenn nachfolgend die Poissongleichung für die Druckkorrektur gelöst wurde. Der Name der Methode leitet sich aus dieser Vorgehensweise ab, bei der der divergenzproduzierende Teil des Strömungsfeldes „heraus projiziert“ wurde [Ferziger \& Peric (1997)]. Die Poissongleichung wird um einen Stabilisierungsterm höherer Ordnung erweitert, damit keine oszillierenden Druckfelder aufgrund entkoppelter benachbarter Druckwerte entstehen.

Die teilimplizite Diskretisierung der Transport- und Quellterme führt zu einer Erweiterung des Stabilitäsbereiches und ermöglicht damit größere Zeitschritte. Dies ist besonders für die hier verwendeten Gitter von Vorteil, da sonst die z.T. extrem kleinen Zellen unmittelbar an der Hinterkante die Zeitschrittweite stark limitieren würden. 
Die Gesamteffizienz eines kompletten Lösungsschrittes wird in hohem Maße vom Lösungsaufwand der Druckkorrekturgleichung (Poissongleichung) des Projektionsschrittes bestimmt. Hier findet die Mehrgittermethode mit einem Jacobi-Löser als Glätter Anwendung. Für die Berechnung der Impuls- und Skalargleichungen wird ein Conjugate-Gradient-, oder ein Bi-CGSTAB- (Bi-Conjugate Gradient Stabilized) oder GMRES-Verfahren (Generalized Minimal Residual) mit Vorkonditionierung verwendet, die Jacobi-Methode steht ebenfalls zur Verfügung, sie ist jedoch gegenüber den anderen Verfahren weniger effektiv.

\subsection{Primäres Rechengitter}

Der Strömungslöser berechnet lokale Größen, z.B. Strömungsgeschwindigkeit und Druck an diskreten Stellen für kleine Kontrollvolumina. Es ist also eine Einteilung des Rechengebietes in Elemente notwendig, d.h. die Generierung eines Rechengitters (Abschnitt 3.2.1). Die dazu verwendbaren Elementarzellen richten sich nach den Anforderungen des Lösers. Es können hybride, dreidimensionale unstrukturierte Gitter verwendet werden. Die einzelnen Kontrollvolumina können prismen-, tetraeder-, hexaeder- und pyramidenförmig sein. Dabei ist darauf zu achten, dass die Lösung in keiner Weise von der Gestalt des Gitters abhängt, damit der simulierte physikalische Prozess nicht von einem numerischen Artefakt überlagert wird. Dies ist nicht nur allgemein, sondern auch durch problemabhängige Validierungen sicherzustellen (vgl. Abschnitt 3.2.3 und Abschnitt 3.2.4).

Ein Kritikpunkt an den Experimenten und damit auch Bestandteil der Motivation zur Durchführung numerischer Simulationen ist die mangelnde räumliche Auflösung von Regionen unmittelbar an der Oberfläche bzw. an der Hinterkante der Platte. Abschnitt 3.2.5 erläutert, dass das verwendete Gitter hier eine besonders gute Diskretisierung bietet.

\subsubsection{Generierung des primären Gitters}

Eine Reihe von Faktoren sind bei der Erzeugung des Gitters zu berücksichtigen, um das Gitter optimal an die Geometrie und das physikalische Problem anzupassen, da dies signifikanten Einfluss auf die Genauigkeit der Lösung haben kann [Kessler (1991)]. Dazu ist es wichtig, dass Regionen, die von besonderem Interesse sind, gut aufgelöst werden, hier z.B. die Hinterkante. Die unmittelbar nach der Anre- 
gung besonders dort auftretenden hohen Gradienten der zu berechnenden Größen müssen durch eine entsprechend feine Diskretisierung ausreichend aufgelöst werden. An den festen Kanalwänden (hier Nord- und Südrand) kann sich die Grenzschicht numerisch nur dann ausbilden, wenn eine bestimmte Anzahl von Gitterpunkten in diesem Bereich liegt (s. auch Abschnitt 3.2.3). Andere Gebiete, wie z.B. der Bereich der Spülströmung, sind von untergeordneter Bedeutung, hier müssen die Gitterpunkte lediglich so dicht verteilt sein, dass es wegen zu großer Abstände nicht zur Divergenz der Lösung kommen kann. Der Übergang von einer fein aufgelösten Region zu einer gröber diskretisierten sollte kontinuierlich sein, um dadurch entstehende Fehler gering zu halten. Ein Gitter, welches diese Kriterien erfüllt ist in Abbildung 3.3 dargestellt.

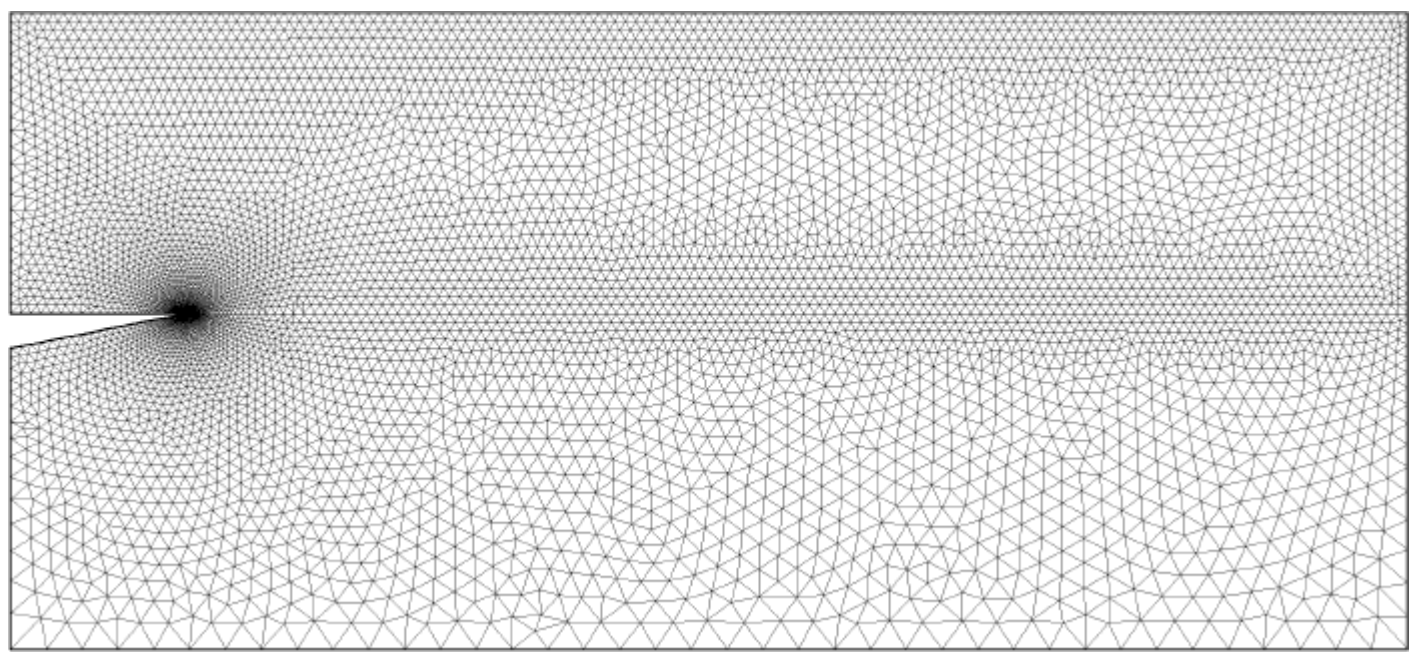

Abbildung 3.3: Schnitt durch die Symmetrieebene eines Gitters

Die untersuchte Strömung wird als zweidimensional behandelt. Es wird daher zunächst ein zweidimensionales primäres Gitter durch Vorgabe der Randpunkte und anschlieBender iterativer Triangulation des Simulationsgebietes erzeugt. Dieses wird dann verdoppelt und bildet als zwei Ebenen hintereinander das dreidimensionale Gitter für den $\tau^{\mathrm{i}}$-Code. Beide Ebenen schließen nach außen mit Symmetrierandbedingungen ab, so dass die Strömungsberechnung quasi zweidimensional erfolgt. Der Abstand der Gitterebenen wurde so gewählt, dass die Distanz zu benachbarten Elementen in der Ebene deutlich kleiner ist, als zu Elementen der zweiten Ebene. Nur so wird eine zweidimensionale Abhängigkeit der Größen gewährleistet. 
Die Begrenzungen des Berechnungsgebietes dürfen keinen Einfluss auf die Strömung im zu untersuchenden Bereich um die Hinterkante haben. Im Einzelnen bedeutet dies, dass der Einströmrand soweit stromauf der Kante gelegen sein muss, dass deren Wirkung die Einströmrandbedingung nicht verletzt (s. Abschnitt 3.2.4). Der Ausströmrand darf zu keinen Reflexionen im Strömungsgebiet führen, d.h. es ist eine geeignete Dämpfung zu verwenden und er sollte mindestens so weit stromab der Kante liegen, dass die erzeugten Störungen während der Beobachtungszeit nicht bis zum Rand konvektieren und das Druckfeld stören können. Eine Aufstellung dieser minimalen Länge $1_{\min }$ des Gitters stromab der Hinterkante ist in (Tabelle 5.1 auf Seite 67) für die verschiedenen simulierten Strömungen zusammengestellt. Die begrenzenden Kanalseitenwände dürfen die Scherschicht nicht beeinflussen, d.h. die Wandgrenzschichten müssen dünn sein gegen die Kanalhöhe.

\subsubsection{Größe der Einströmränder}

Der Delta-Druckgradientenimpuls, bzw. das dadurch erzeugte Potenzialfeld, wird durch instationäre Randbedingungen simuliert (vgl. Abschnitt 4.2.1 auf Seite 46). Auf das Einströmprofil der Hauptströmung wird zum Zeitpunkt $t=0$ ein Kastenprofil addiert und genauso von der Nebenströmung subtrahiert. Dies entspricht am Einströmrand der Überlagerung mit einer rotationsfreien Potenzialströmung. Sind nun die Einströmränder von Haupt- und Spülströmung verschieden groß, so entsteht ein Massenfluss in das Berechnungsgebiet hinein, der am Ausströmrand ausgeglichen werden muss, damit die notwendige Bedingung der Divergenzfreiheit des Randintegrals und damit die Kontinuitätsgleichung wieder erfüllt ist. Das Programm multipliziert dazu das Ausströmprofil mit einem Faktor, was bei i.a. nicht konstanten Geschwindigkeiten mit der Erzeugung von Wirbeligkeit am Ausströmrand gleichbedeutend ist. Um also der Forderung nach Rotationsfreiheit der überlagerten Strömung gerecht zu werden, ist die Ausströmrandbedingung zu ändern, oder aber dafür Sorge zu tragen, dass durch die zeitabhängige instationäre Anregung kein auszugleichender Massenfluss entsteht. Letzteres kann dadurch realisiert werden, dass die gleiche Anregungsfunktion (mit anderem Vorzeichen) für die beiden Einströmränder verwendet wird und beide Gebiete gleich groß sind. In Anlehnung an einen Wasserkanal, in dem Experimente durchgeführt wurden, ist die Höhe der Einströmränder jeweils $8.6^{*} 10^{-2} \mathrm{~m}$. 


\subsubsection{Anforderungen der Einströmrandbedingungen an das Gitter}

Die Anregung führt zu einem sehr hohen Gradienten der u-Komponente der Geschwindigkeit unmittelbar an der Wand. Um diesen Gradienten ausreichend aufzulösen, wurde eine Gitterstudie durchgeführt. Es wurde ein Kanal mit den Abmessungen $0.1 \mathrm{~m} \times 0.1 \mathrm{~m} \times 0.1 \mathrm{~m}$ gerechnet, der mit gleichförmigen Elementen diskretisiert wurde, d.h. im Innern des Simulationsgebietes waren die Zellen ebenso groß wie am Rand. Es zeigte sich, dass die Abweichungen der Strömungsgeschwindigkeit von der Anregungsfunktion zum Rand hin zunehmen, daher wurde die Daten, gewonnen an einem Monitorpunkt, unmittelbar über der Wand analysiert. Die ersten Zelle des gröbsten Gitters ist ca. $2 * 10^{-3} \mathrm{~m}$ vom Rand entfernt; diese Position wurde zur besseren Vergleichbarkeit für alle Gitter gewählt. In den anderen Raumrichtungen befindet sich der Monitorpunkt in der Mitte des Berechnungsgebiets, um den Einfluss des Einström- und des Ausströmrandes möglichst gering zu halten.

Die Reproduzierbarkeit der Einströmfunktion (vgl. Abschnitt 4.2.1 auf Seite 46) in Abhängigkeit der Zellkantenlänge von drei verschiedenen Gittern ist in Abbildung 3.4 dargestellt. Für das Gitter mit $2 * 10^{-3} \mathrm{~m}$ Elementweite ist die Abweichung vom vorgegebenen Wert ca. $5 \%$, für das mit $1 * 10^{-3} \mathrm{~m}$ unter $2 \%$ und für das Gitter mit $5 * 10^{-4} \mathrm{~m}$ Elementweite unter 1\%. Die Verwendung von Zellen mit einer Kantenlänge von mehr als $1 * 10^{-3} \mathrm{~m}$ führt somit zu einer ungenauen Abbildung der Anregungsfunktion und ist aus diesem Grund nicht sinnvoll.

Die gezeigten Simulationsergbnisse wurden mit einer Anregungsgeschwindigkeit von $5^{*} 10^{-5} \mathrm{~m} / \mathrm{s}$, einer Viskosität von $10^{-6} \mathrm{~m}^{2} / \mathrm{s}$ und einem Zeitschritt von $0.005 \mathrm{~s}$ berechnet. Weiter Vergleiche haben gezeigt, dass eine Variation der Viskosität kaum Einfluss auf die Genauigkeit hat und gute Resultate für Zeitschritte von $0.01 \mathrm{~s}$ oder kleiner erhalten werden können. Zur Wahl des Zeitschrittes s. auch Abschnitt 5 auf Seite 54.

\subsubsection{Vorlauf der Strömung bis zur Kante}

Die Wirkung der Kante stromauf muss bis zum Einströmrand vernachlässigbar klein sein, da dort die Randwerte für eine parallele Kanalströmung vorgegeben werden. Um zu prüfen, wie lang der dazu nötige Vorlauf der Strömung bis zur Kante sein muss, wurde die zeitliche Entwicklung der u-Komponente an verschiedenen Monitorpunkten mit der analytischen Einströmfunktion verglichen. Die Monitorpunkte lagen wie in 


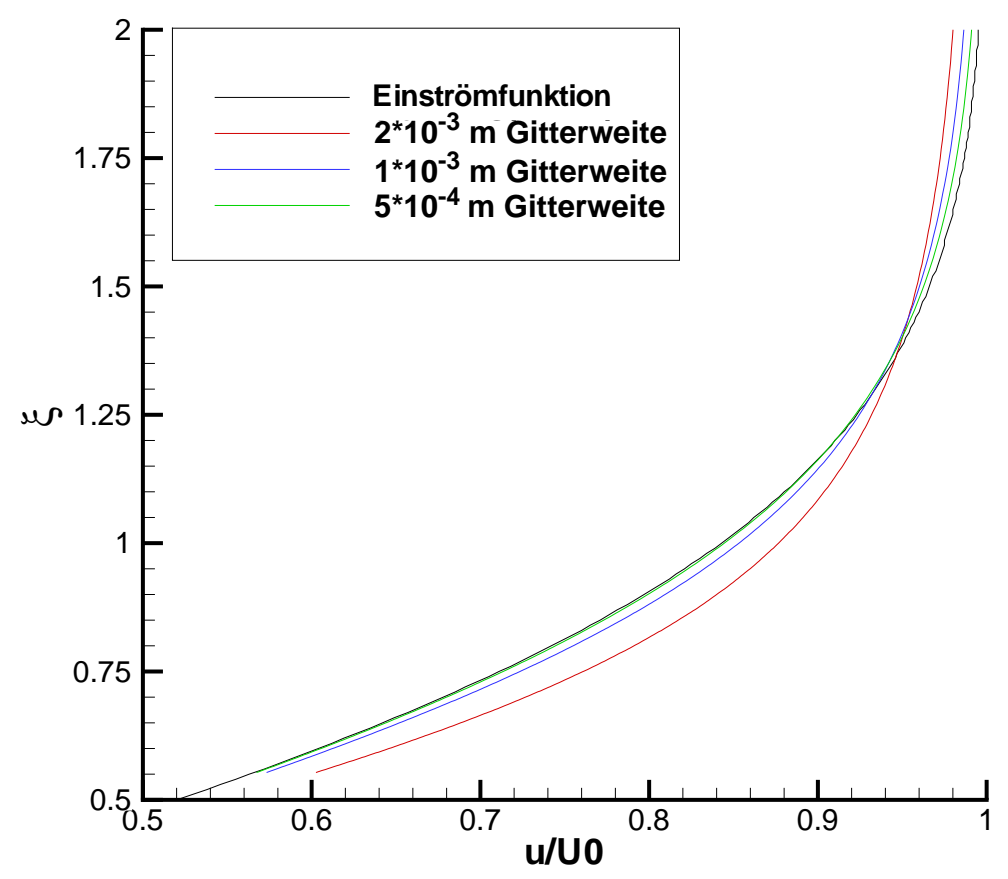

Abbildung 3.4: Vergleich der Reproduzierbarkeit der Einströmfunktion für verschieden feine Gitter

Abschnitt 3.2.3 $2 * 10^{-3} \mathrm{~m}$ über der Platte und in verschiedenen Abständen $\left(1 * 10^{-2} \mathrm{~m}\right.$, $2 * 10^{-2} \mathrm{~m}$ und $3 * 10^{-2} \mathrm{~m}$ ) stromab vom Einströmrand. Abbildung 3.5 zeigt die Reproduzierbarkeit der am Rand vorgegebenen Werte im Strömungsfeld für eine Simulation mit $0.1 \mathrm{~m}$ Vorlauf bis zur Kante. Deren Einfluss macht sich in einem Wachstum der Abweichungen stromab des Einströmrandes bemerkbar. Hier ist der Abstand der Hinterkante vom Einströmrand zu gering, so dass es bis unmittelbar vor den Rand noch zu Störungen der u-Komponente kommt. Die Vorgabe der Randwerte führt hier zu einer unnatürlichen, gezwungenen Strömung.

Bei der Simulation mit 0.15 m Vorlauf bis zur Kante wird die zeitliche Entwicklung ungestört ins Feld transportiert. Abbildung 3.6 zeigt, dass nahezu keine Abweichung von den vorgegebenen Werten bis zum Monitorpunkt $3 * 10^{-2} \mathrm{~m}$ stromab des Einströmrandes zu erkennen sind. Für die folgenden Simulationen ist somit ein Abstand KanteEinströmrand von mindestens 0.15 m gewählt worden.

Die Erhöhung der Viskosität dämpft die Stromaufwirkung der Kante, jedoch nicht so stark wie ein gröberes Gitter. Die Elemente über der Platte des in dieser Studie verwendeten Gitters hatten eine Kantenlänge von $1 * 10^{-3} \mathrm{~m}$. 


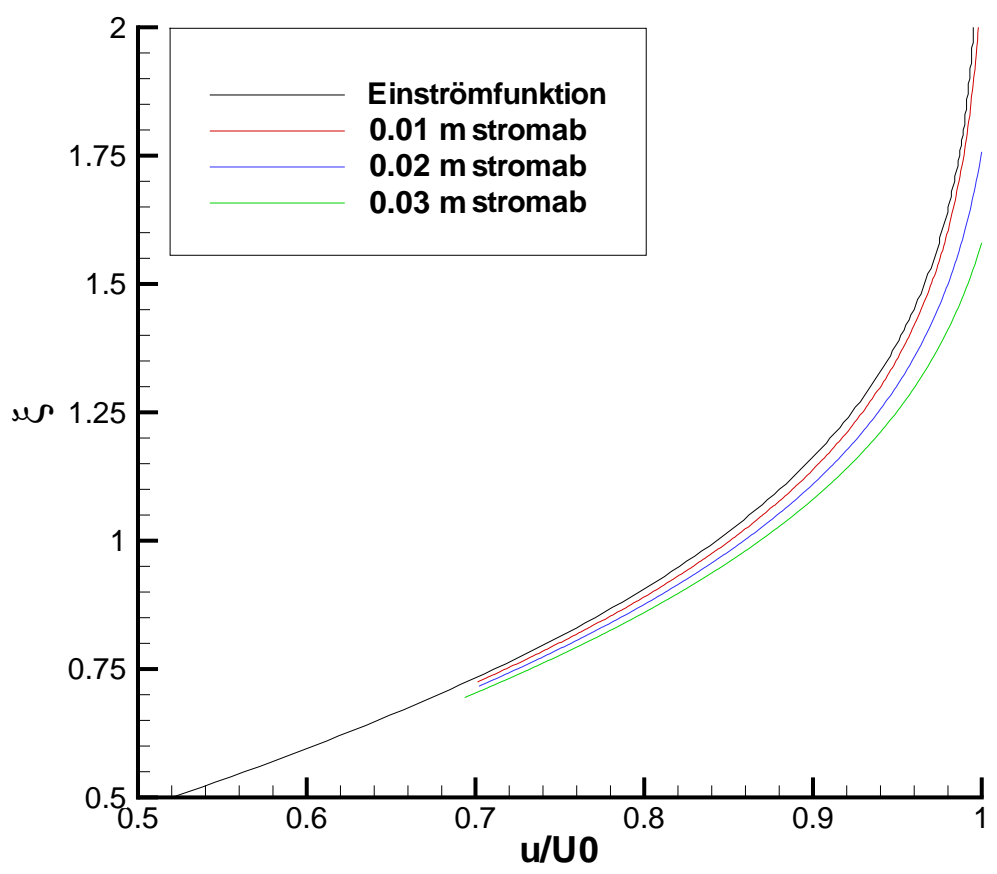

Abbildung 3.5: Abweichung von der Einströmfunktion der u-Komponente an unterschiedlichen Positionen stromab des Einströmrandes für eine Simulation mit 0.10 m Vorlauf bis zur Kante

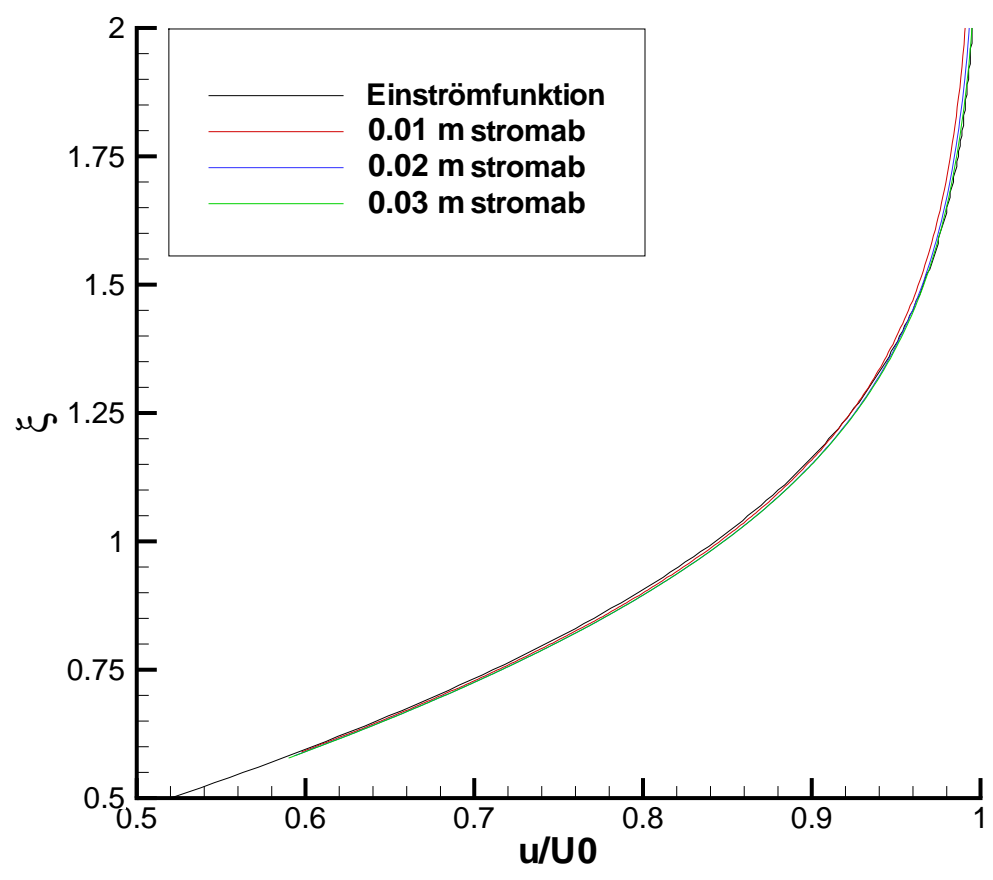

Abbildung 3.6: Abweichung von der Einströmfunktion der u-Komponente an unterschiedlichen Positionen stromab des Einströmrandes für eine Simulation mit 0.15 m Vorlauf bis zur Kante 


\subsubsection{Kantenregion}

Besonders an der Hinterkante der Platte muss das Gitter sehr fein sein, da bei der Umströmung dort lokal hohe Gradienten numerisch aufgelöst werden müssen. Hierzu wurde ein Rechengitter verwendet, welches eine deutlich bessere räumliche Auflösung aufweist, als dies im Experiment möglich war. Die ortsaufgelöste Messung der Strömungsgeschwindigkeiten erfolgte mit einem Laser-Doppler-Anemometer (LDA). Neben vielen Vorzügen, die diese berührungsfreie Messtechnik bietet, wie z.B. deren guter zeitlicher Auflösung, ergeben sich aus den optischen Anforderungen an den Aufbau des Experiments auch Limitierungen. Das Messvolumen, gebildet aus dem Schnittgebiet zweier Laserstrahlen, hat eine endliche Ausdehnung von $2 * 10^{-4} \mathrm{~m}$. Es ist daher nicht möglich, beliebig dicht über der Platte die Strömungsgeschwindigkeit zu bestimmen. Mit dem LDA wird das Streulicht von Partikeln ausgewertet, die dem Fluid zugesetzt sind, wenn sie das Messvolumen durchqueren. Damit ergab sich die Schwierigkeit, dass gerade in der Grenzschicht u.a. aufgrund der geringen Strömungsgeschwindigkeit und der wenigen Streuteilchen die Messung erschwert wurde. Ein Mindestabstand von der Oberfläche der Platte war auch nötig, weil das Streulicht von Partikeln dort reflektiert wurde und somit nicht mehr eindeutig auszuwerten war. Gute Ergebnisse wurden für Punkte mindestens $2 * 10^{-4} \mathrm{~m}$ von der Platte entfernt erzielt. Abbildung 3.7 zeigt das für das LDA unzugängliche Gebiet von $2 * 10^{-4} \mathrm{~m} \mathrm{x} 2 * 10^{-4} \mathrm{~m}$ um die Hinterkante. Der Beitrag dieses Bereichs zu der untersuchten Dynamik kann in der Simulation somit vollständig erfasst werden.

Die hohe Auflösung der Hinterkante ermöglicht den Vergleich zwischen einer eckigen und einer runden Kante. Die beiden Kantenformen und die Maxima der entstehenden Wirbelstärke sind in Abbildung 3.8 dargestellt. An der eckigen Kante entspricht die Wirbeligkeitsstruktur nicht der im Modell postulierten punktförmigen Quelle, an der abgerundeten Hinterkante ist dies gut erfüllt.

Die obige Betrachtung bezieht sich auf einen sehr kleinen und lokal begrenzten Bereich. Im Fernfeld der Kante konnte von der Wirbeligkeitsverteilung nicht auf die Kantengeometrie zurückgeschlossen werden. Da aber die Modellierung der Quelle einer der Wirbelstärke vergleichbaren Transportgröße besonders von den Maxima der 


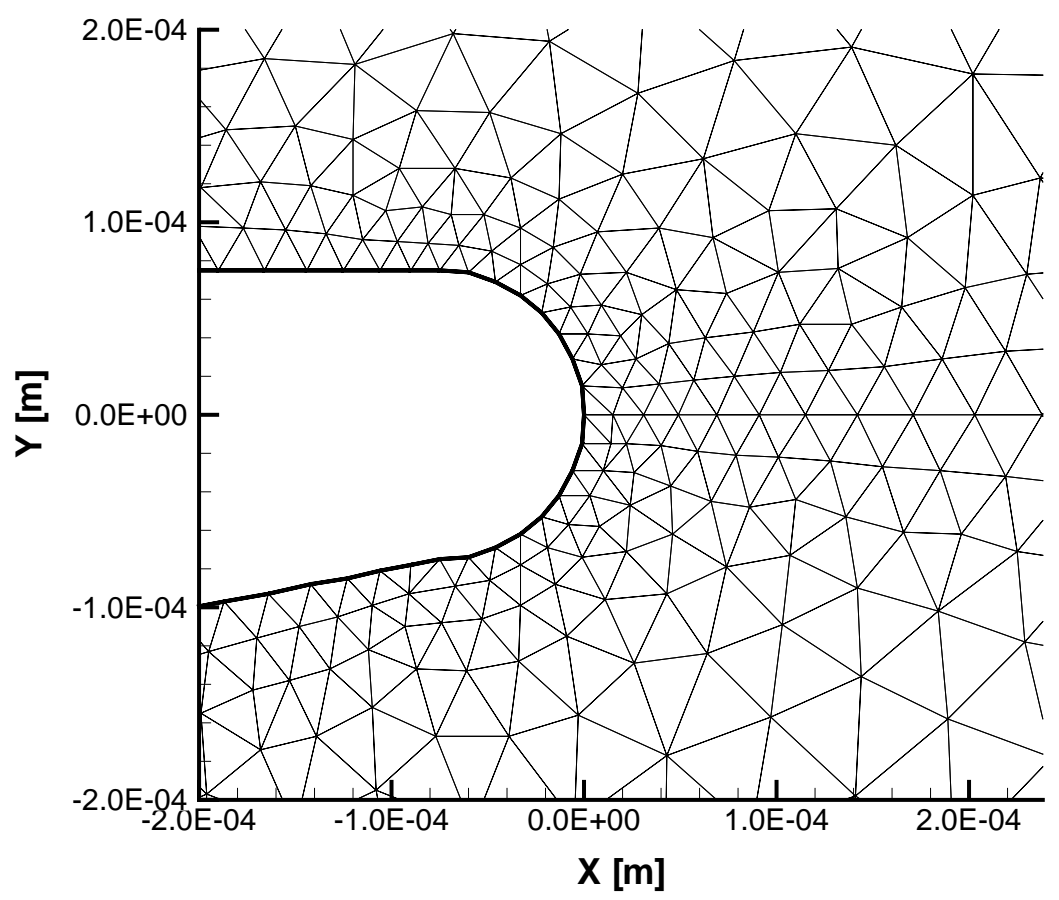

Abbildung 3.7: Gitter in einem Bereich von $4^{*} 10^{-4} \mathrm{~m} \mathrm{x} 4^{*} 10^{-4} \mathrm{~m}$ um die Hinterkante

Wirbeligkeit an der Kante abhängt, ist die abgerundete Kante zweifellos von Vorteil. Im Folgenden werden daher ausschließlich Simulationen mit runder Hinterkante verwendet.

\subsection{Berechnung der Wirbelstärke am Rand}

Für die inneren Punkte des Strömungsfeldes kann die Wirbeligkeit aus den Gradienten $(\partial u) /(\partial y)$ und $(\partial v) /(\partial x)$ berechnet werden. Diese lassen sich im Feld aus den Werten der Variablen an Nachbarelemente ermitteln. Für Punkte auf dem Rand des Berechnungsgebietes ist die Bestimmung der Gradienten auf diese Weise nicht möglich, da zu mindestens einer Seite Nachbarpunkte fehlen. Wird dies bei der Berechnung der Wirbelstärke nicht berücksichtigt, so kann es zu signifikanten Fehlern kommen. Abbildung 3.9 zeigt den zeitlichen Verlauf des Oten Momentes der Wirbeligkeit (zur Berechnung s. Anhang, Abschnitt A.1 auf Seite 87), entstanden durch Anregung eines ruhenden Fluids, wie in Abschnitt 4.2.1 auf Seite 46 beschrieben. Entgegen dem zu erwartenden sprunghaften Anwachsen des Momentes findet man ein kontinuierliches Wachstum, welches auch nach Sekunden bzw. nach mehreren hundert Iterationen noch keinen Sättigungszustand erreicht hat. Zu erklären ist dieses Verhalten aus der besonderen Behandlung der Randzellen des Berechnungsgebietes. 

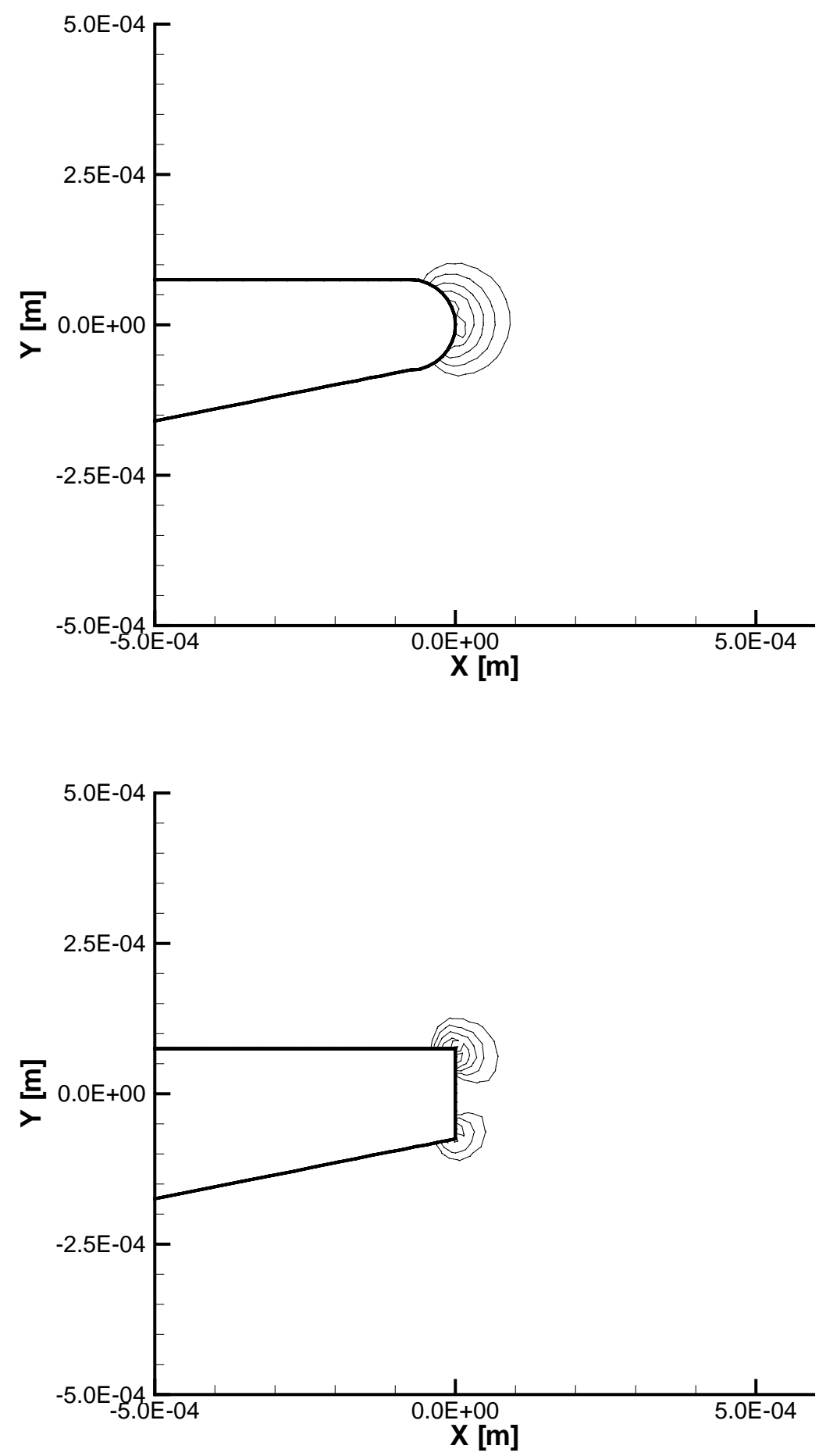

Abbildung 3.8: Zum Vergleich der Kantenformen sind gleiche Isolinien der Wirbeligkeit dargestellt.

Ausgehend vom primären Gitter kann am Rand nur eine halbe Zelle abgegrenzt werden. Die Bilanzen über derartige Elemente müssten für einen Punkt auf dessen Rand gebildet werden, was zu numerischen Fehlern führen kann. Daher wird der ursprüngliche Randpunkt nach innen verschoben, wie in Abbildung 3.10 gezeigt. 


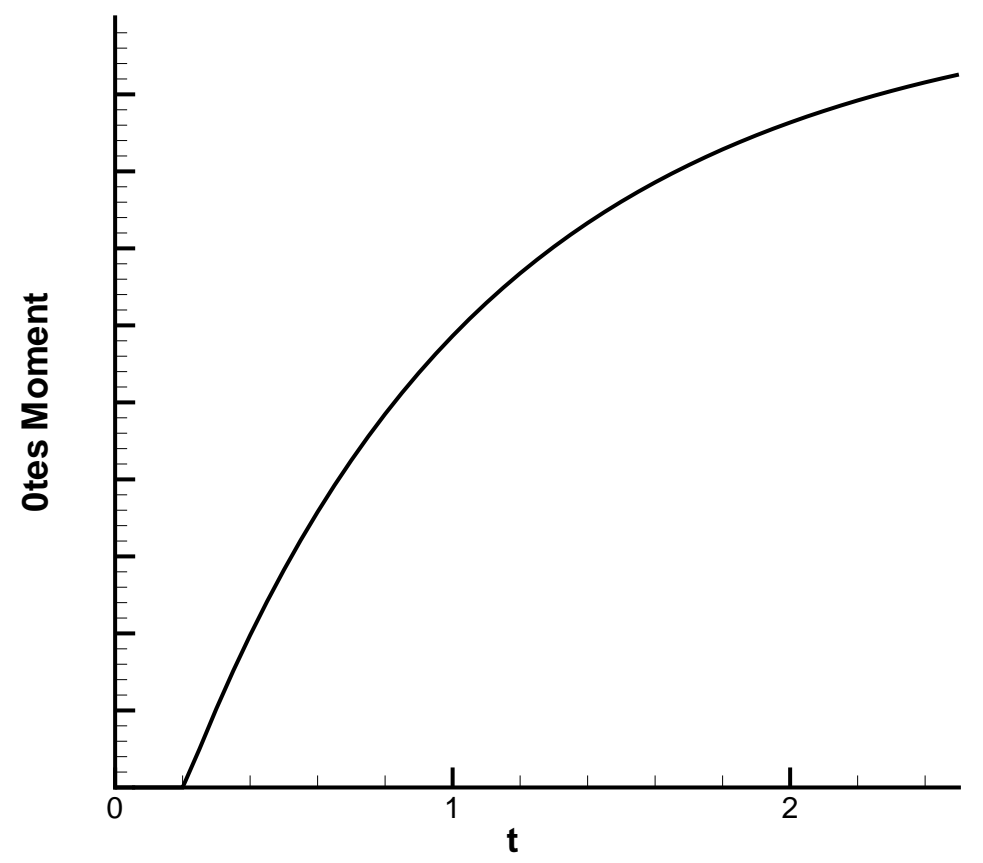

Abbildung 3.9: Zeitlicher Verlauf des Oten Momentes der Wirbeligkeit $4.5^{*} 10^{-2} \mathrm{~m}$ stromauf der Kante, berechnet ohne besondere Behandlung der Gebietsgrenzen

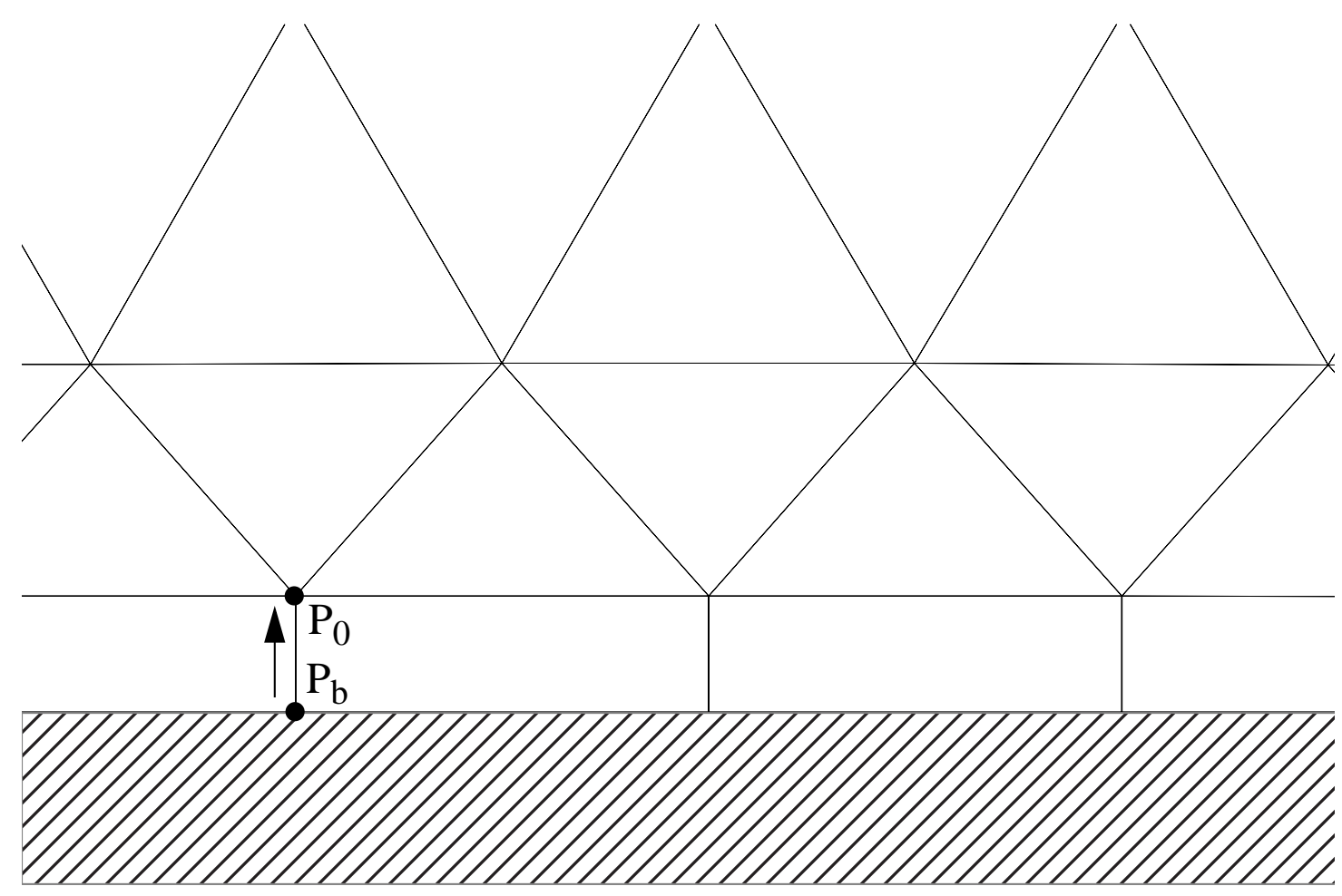

Abbildung 3.10: Zellstruktur am Rand des Rechengebietes 
Um den neu entstandenen Punkt $\mathrm{P}_{0}$ kann eine vollständige Zelle gebildet werden. Die Variablen in $\mathrm{P}_{\mathrm{b}}$ werden durch geeignete Randbedingungen gesetzt und müssen nicht berechnet werden.

Für die eigentlichen Berechnung sind nun die ursprünglichen Randpunkte innere Punkte, daher werden die Werte an den (zusätzlichen) Randpunkte, wie $\mathrm{P}_{\mathrm{b}}$, vor der Datenausgabe auf die ursprünglichen Randpunkte, wie $\mathrm{P}_{0}$, kopiert und deren Werte damit überschrieben. In der Ausgabe stimmen also die Position, an der die Werte berechnet bzw. gesetzt wurden und an der sie dargestellt werden wieder überein. Wie bereits erwähnt, kann die Wirbeligkeit aufgrund fehlender Gradienten am Rand nicht berechnet werden, ihr Wert in $\mathrm{P}_{0}$ wird daher auch nicht überschrieben.

Die durch die Anregung erzeugte Wirbeligkeit konzentriert sich zunächst auf eine infinitesimal dünne Schicht an der Wand, die erst mit fortschreitender Zeit wächst. Ohne eine gesonderte Bestimmung der Wirbelstärke unmittelbar an der Wand geht für die Berechnung des Momentes ein bedeutender Anteil der erzeugten Wirbeligkeit verloren. Mit sinkendem Gradienten $(\partial u) /(\partial y)$ wird die nicht berücksichtigte Wirbelstärke kleiner und das Moment steigt, wie in Abbildung 3.9 gezeigt.

Die Berechnung der Wirbelstärke am Rand nach [Kessler, private Mitteilungen] erfolgt ähnlich der Bestimmung der Wandschubspannung. Sie ist im Folgenden kurz skizziert.

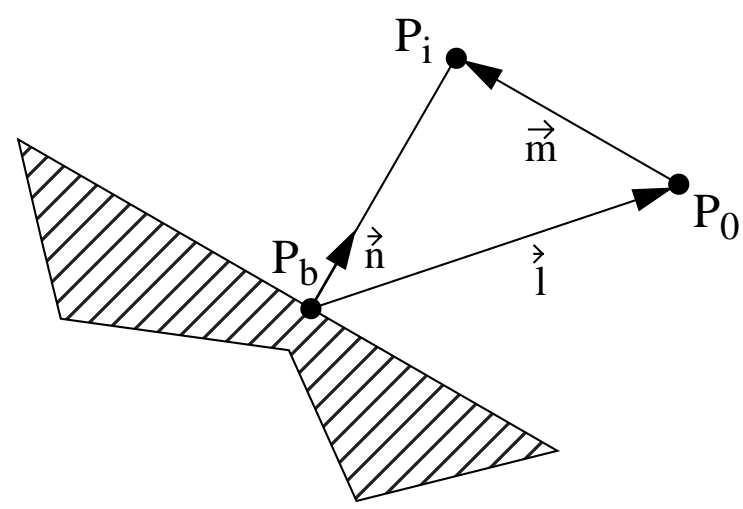

Abbildung 3.11: Zur Berechnung der Wirbelstärke am Rand notwendige Punkte 
Die Wirbelstärke im Punkt $\mathrm{P}_{\mathrm{b}}$ auf dem Rand wird in einem wandnormalen Koordinatensystem berechnet. Es gilt:

$$
\omega=\frac{\partial \mathrm{u}_{\mathrm{t}}}{\partial \mathrm{n}_{0}}-\frac{\partial \mathrm{u}_{\mathrm{n}}}{\partial \mathrm{t}_{0}}
$$

Wobei $u_{t}$ die wandtangentiale und $u_{n}$ die wandnormale Komponente der Geschwindigkeit ist und $\mathrm{t}_{0}$ die wandtangentiale und $\mathrm{n}_{0}$ die wandnormale Richtung ist. Da sich in einer festen Wand keine Quellen oder Senken befinden, ist $\frac{\partial u_{n}}{\partial t_{0}}=0$ und somit liefert nur der tangentiale Anteil der Strömungsgeschwindigkeit einen Beitrag zur Bestimmung der Wirbeligkeit. Um den Gradienten der Geschwindigkeiten in Richtung der Wandnormalen $\vec{n}$ berechnen zu können, muss die Differenz der Geschwindigkeiten im Punkt $\mathrm{P}_{\mathrm{b}}$ und $\mathrm{P}_{\mathrm{i}}$ bestimmt werden. In $\mathrm{P}_{\mathrm{b}}$ sind die Werte bekannt und in $\mathrm{P}_{\mathrm{i}}$ lassen sie sich aus den Werten in $\mathrm{P}_{0}$ und seiner Lage zu $\mathrm{P}_{\mathrm{b}}$ bestimmen. Für $\overrightarrow{\mathrm{u}}$ im Punkt $\mathrm{P}_{\mathrm{i}}$ gilt:

$$
\left.\overrightarrow{\mathrm{u}}\right|_{\mathrm{i}}=\left.\overrightarrow{\mathrm{u}}\right|_{0}+\left.\overrightarrow{\mathrm{m}} \nabla \overrightarrow{\mathrm{u}}\right|_{0}
$$

Die Tangentialkomponente der Geschwindigkeiten findet man nach:

$$
\left.u_{t}\right|_{i}=\frac{\left.\vec{u}\right|_{i} \times \vec{n}}{|\vec{n}|}
$$

Damit folgt für die Ableitung normal zur Wand:

$$
\frac{\partial \mathrm{u}_{\mathrm{t}}}{\partial \mathrm{n}_{0}} \approx \frac{\left.\mathrm{u}_{\mathrm{t}}\right|_{\mathrm{i}}-\left.\mathrm{u}_{\mathrm{t}}\right|_{\mathrm{b}}}{\mathrm{d}}
$$

wobei d der Abstand zwischen $\mathrm{P}_{\mathrm{b}}$ und $\mathrm{P}_{\mathrm{i}}$ ist, gemäß:

$$
\mathrm{d}=\overrightarrow{1} \cdot \frac{\overrightarrow{\mathrm{n}}}{|\overrightarrow{\mathrm{n}}|}
$$

Wird die auf diese Weise berechnete Wirbeligkeit am Rand des Berechnungsgebietes mitberücksichtigt, so ergibt sich der in Abbildung 3.12 gezeigte zeitliche Verlauf des Oten Momentes der Wirbeligkeit, der nahezu einer Sprungfunktion entspricht. Das Ote Moment ist unmittelbar nach der Anregung zu groß. Dies resultiert aus der Umströ- 
mung der Kante und der damit verbundenen Beschleunigung der Strömung (vgl. auch Abbildung 4.11 auf Seite 50). Das Moment sinkt dann mit der Zeit auf den erwarteten Wert ab.

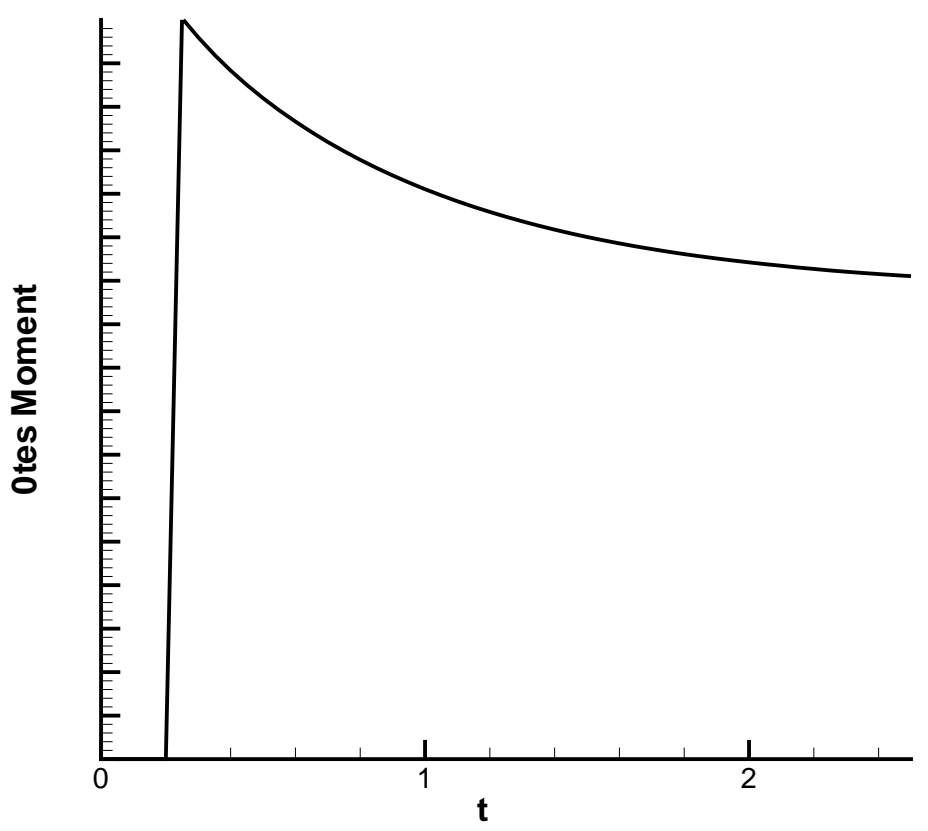

Abbildung 3.12: Zeitlicher Verlauf des Oten Momentes der Wirbeligkeit $4.5^{*} 10^{-2} \mathrm{~m}$ stromauf der Kante, berechnet unter besonderer Behandlung der Gebietsgrenzen

\subsection{Numerische Stabilität der Scherschicht}

Bei den durchgeführten Simulationen trat eine besondere Art der Wechselwirkung zwischen den physikalischen Eigenschaften einer Scherschicht und deren numerischer Approximation auf. Nach experimentellen Beobachtungen [Ronneberger, private Mitteilung] und stabilitätstheoretischen Überlegungen [u.a. Koch, private Mitteilung] ist zu erwarten, dass die Scherschicht konvektiv instabil ist, d.h. eingebrachte Störungen schwimmen bei räumlichem Wachstum stromab. Treten keine Störungen auf, so entwickelt sich eine stationäre Strömung.

Betrachtet man die Residuen einer Simulation als Maß für die Erfüllung der Kontinuitätsgleichung bzw. als Maß für die zeitliche Ableitung, so traten zwei charakteristische Szenarien auf. Ausgehend von einem Anfangszustand, bei dem die Kontinuitätsgleichung nur unzureichend erfüllt ist, sinken die Residuen bis auf ein Niveau, welches der 
Lösung der Gleichungen bei Maschinengenauigkeit entspricht. Dies ist in Abbildung 3.13 a) dargestellt. Hier treten physikalisch keine zeitlichen Änderungen auf, d.h. die Kontinuität ist in jedem Zeitschritt gleichermaßen erfüllt.

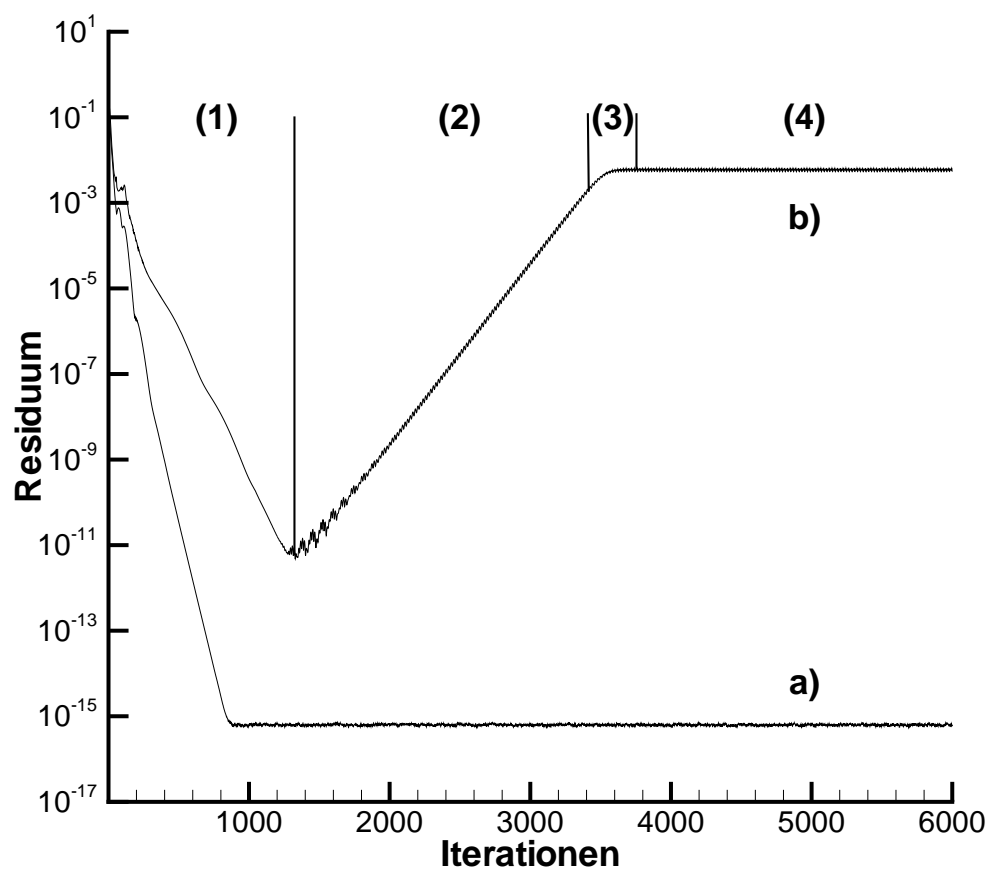

Abbildung 3.13: Verlauf der Residuen der Simulation einer stabilen Scherschicht (a) und einer instabilen Scherschicht (b). Dort lasssen sich drei verschiedene Phasen unterscheiden: (1) Entwicklung zum quasistationären Zustand; (2) linearer Wachstumsbereich; (3) Übergangsbereich; (4) Sättigungszustand

Häufig entwickelt sich der in Abbildung 3.13 b) gezeigte Residuenverlauf, den auch Hannemann (1988) bei der Untersuchung eines absolut instabilen Nachlaufs fand und in vier charakteristische Bereich einteilte. Die Entwicklung zum quasistationären Zustand (1) ist ebenso wie für die stationäre Strömung durch ein Absinken der Residuen über mehrere Größenordnungen gekennzeichnet. Aus dem Anfangsrandwertproblem entwickelt sich eine Scherschicht, die eine konsistente Lösung repräsentiert. Diese ist sehr rezeptiv für Störungen, die z.B. durch die Diskretisierung oder ungünstige numerische Randbedingungen entstehen können. Das Vorhandensein dieser Einflüsse äußert sich dann im linearen Wachstumsbereich (2), während dessen die quasistationäre Strömung zunehmend instabil wird und deren Zeitableitung zunimmt. Nach einem Übergangsbereich (3) stellt sich der Sättigungszustand (4) ein, bei dem die Instabilität voll ausgebildet ist. 
Oft können für das jeweilige Problem ungeeignete Gitter sowie darauf nicht abgestimmte Zeitschritte einen numerischen Algorithmus zu Oszillationen anregen. Bei der Simulation instationärer Strömungen können dann tatsächlich physikalisch bedingte und künstliche Schwingungen nicht mehr unterschieden werden. So zeigen die Strömungsfelder im Sättigungszustand alle Merkmale einer globalen Instabilität. Exemplarisch sind in Abbildung 3.14 die Isolinien des Drucks dargestellt. Man erkennt das räumliche Wachstum und die Anfachung der Instabilität. Eine zeitliche Serie von Simulationsergebnissen zeigte die Konvektion der Störung und damit ein weiteres physikalisches Merkmal einer global instabilen Scherschicht.

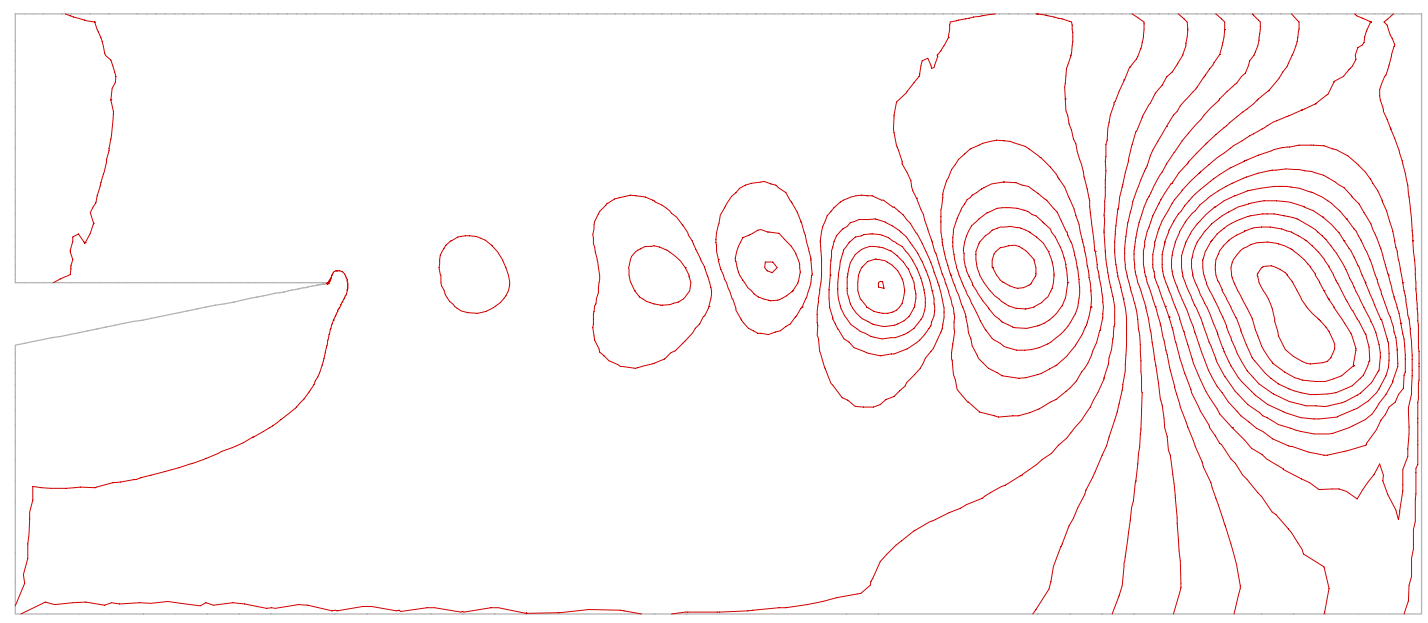

Abbildung 3.14: Isolinien des Drucks im Sättigungszustand

In Gitter- und Zeitschrittstudien wurden Parameterkombinationen ermittelt, die den Einfluss dieses Phänomens dämpften, nicht jedoch auslöschen konnten. Daher ist es naheliegend, dass eine Störung, entstanden am Ausströmrand, die Instabilität anfacht und aufrechterhält. Eine Möglichkeit, die Scherschicht zu stabilisieren, ist die Verwendung einer numerisch nicht reflektierenden Ausströmrandbedingung. Deren Eigenschaften sind jedoch sehr eng mit den Algorithmen des Strömungslösers verknüpft, so dass sie sich nicht ohne aufwendige Anpassungen und Tests von einem Programm, z.B. aus [Colonius (1996)], in ein anderes, hier den $\tau^{\mathrm{i}}$-Code, übertragen lassen. Es wurden daher verschiedene Modifikationen der implementierten Randbedingung getestet. Diese erwiesen sich jedoch als nicht vollkommen numerisch reflexionsfrei und konnten die Stabilität der Scherschicht nicht wesentlich erhöhen. 
Für die in dieser Arbeit beschriebenen Simulationen ist die Instabilität mit keinen wesentlichen Einschränkungen verbunden. Z.B. kann die Viskosität erhöht werden, d.h. die Scherschicht ist stabiler und Störungen werden stärker gedämpft. In diesem Fall findet man den in Abbildung 3.13 a) dargestellten Residuenverlauf. Ist dies nicht möglich, so ist der quasistationäre Zustand als Startlösung für die Simulation von Anregung und Relaxation der Scherschicht sehr gut verwendbar. Auch hier sind die Residuen um mehrere Größenordnungen gesunken, und die Strömung ist voll ausgebildet. Wie verschiedene Tests gezeigt haben, ist ihre zeitliche Änderung im Vergleich zu den eingebrachten Störungen vernachlässigbar. Einzige Anforderung an die Länge des Berechnungsgebietes ist in diesem Fall, dass die aufgrund der Anregung entstandene Störung innerhalb der Beobachtungszeit nicht bis zum Ausströmrand gelangt und dort eine Druckstörung initiiert, die instantan auf das gesamte Strömungsfeld wirkt. 


\section{Charakterisierung der Strömungsfelder}

Die Auslenkung der Scherschicht wird dadurch realisiert, dass einer Gleichströmung eine Potenzialströmung überlagert wird. Die beiden Strömungsfelder können deswegen zunächst unabhängig voneinander betrachtet werden. Ihre Entstehung wird ausschließlich von Randbedingungen beeinflusst, die ebenfalls voneinander unabhängig sind. Diese sind entscheidend bei der Übertragung der zu untersuchenden Fragestellung von der Realität in die Simulation. Ein anfangs (mit Null) initialisiertes Strömungsfeld wird sich gemäß der am Rand vorgegebenen ggf. zeitabhängigen Werte nach den implementierten Gesetzmäßigkeiten entwickeln. Das Ergebnis wird somit durch Lösen eines Anfangs-Randwertproblems erhalten. Ein Fehler am Rand kann sich daher im gesamten Berechnungsgebiet ausbreiten, so dass gerade die stationären (Abschnitt 4.1.1) und die instationären Einströmrandbedingungen (Abschnitt 4.2.1) sorgfältig gesetzt werden müssen. In Abschnitt 4.1 werden darüber hinaus die Charakteristika der Gleichströmung und in Abschnitt 4.2 die der überlagerten Strömung beschrieben. Die Eigenschaften der resultierenden instationären Scherschicht-Auslenkung werden von den Merkmalen beider elementarer Felder bestimmt und sind Gegenstand der folgenden Kapitel. Abschnitt 4.3 beschreibt den zu erwartenden Endzustand nach vollständiger Relaxation der Strömung.

\subsection{Stationäre Gleichströmung}

Ausgangssituation für die Anregung einer Scherschicht ist eine stationäre Gleichströmung. Bei der Überströmung der Splitterplatte bildet sich eine Grenzschicht aus, die nach Ablösen von der Hinterkante in eine freie Scherschicht übergeht. Die verschiedenen Parameter dieser stationären Strömung, wie Impulsverlustdicke $\Theta$ und Geschwindigkeit der Kernströmung $U_{\infty}$, sind auch für die Dynamik der instationären Scherschichtablösung relevant.

In Anlehnung an das Experiment [Graf (1998)] wurde eine Gleichströmung berechnet, die als Startlösung für die Simulation der instationären Anregung dient. Hierzu waren mehrere Teilschritte notwendig, um den numerischen Aufwand zu reduzieren. Diese sind in Abbildung 4.1 skizziert. Die Vorgehensweise dient im Wesentlichen dazu, 
geeignete Einströmrandbedingungen zu definieren; dies ist in Abschnitt 4.1.1 weiter erläutert. Die Eigenschaften der daraus resultierenden Gleichströmung sind in Abschnitt 4.1.2 beschrieben.

a) Simulation einer Kanalströmung (stationäre Hauptströmung)

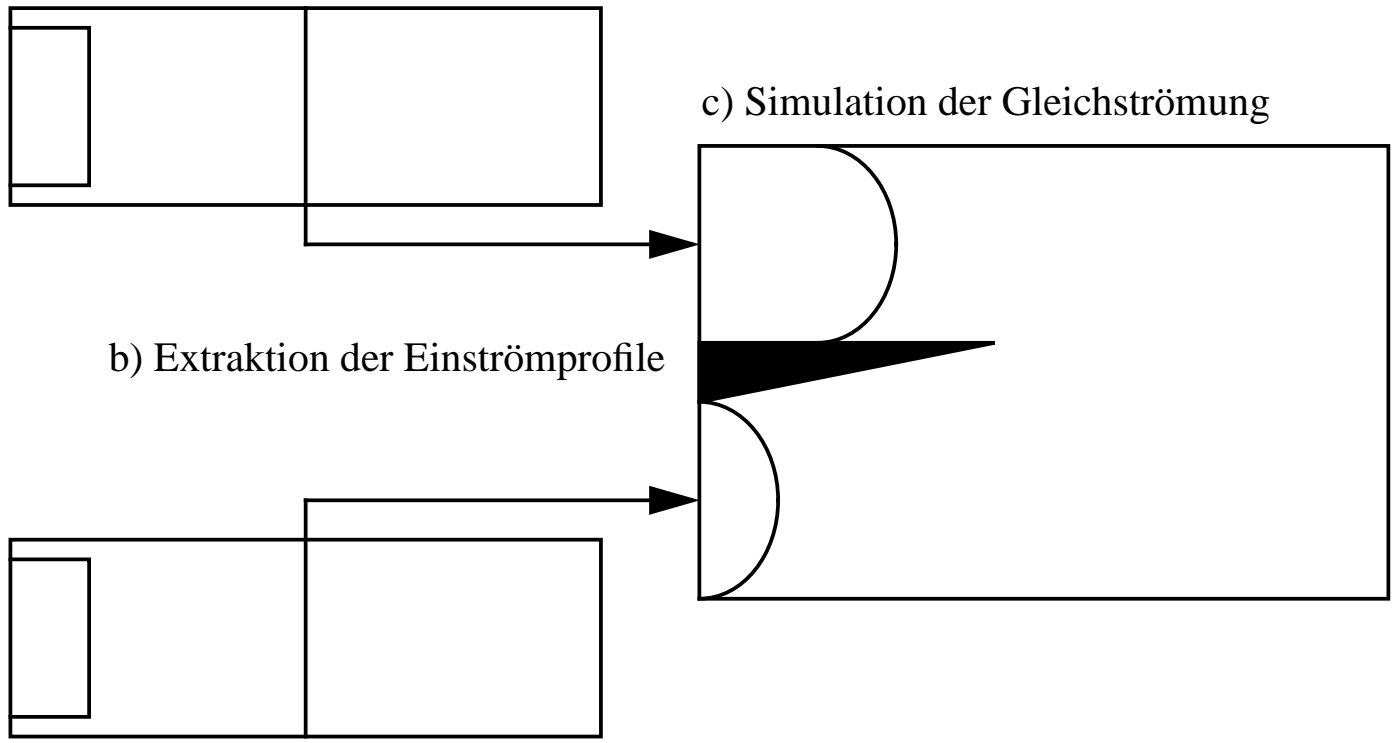

a) Simulation einer Kanalströmung (stationäre Nebenströmung)

Abbildung 4.1: Blockdiagramm der Teilschritte zur Simulation der stationären Gleichströmung: a) getrennte Simulation von Haupt- und Spülströmung b) Extraktion von Strömungsprofilen, die dann als Einströmrandbedingung für die Berechnung der eigentlichen Gleichströmung über die Platte (c) dienen

\subsubsection{Stationäre Einströmrandbedingungen}

Für die Ausbildung der zu untersuchenden Scherschicht ist die Form der Plattengrenzschicht stromauf der Hinterkante maßgeblich. Als Einströmprofil für die numerische Simulation der Splitterplatte (Abbildung $4.1 \mathrm{c}$ ) sollte daher eine möglichst exakte Nachbildung der Grenzschicht, wie sie sich im Experiment entwickelt, verwendet werden. Im Wasserkanal hat die Strömung nach einem Vorlauf von $0.9 \mathrm{~m}$ die Hinterkante erreicht. Für die Simulation der Messstrecke ist dieser Vorlauf nicht zwingend erforderlich, da ein Grenzschichtprofil am Einströmrand vorgegeben werden kann.

Nutzbar hierfür sind Messungen der Geschwindigkeitskomponenten, die über den Rand der Grenzschicht hinaus, in die freie Strömung reichen. In [Graf (1998)] finden sich Werte für ein vollständiges Profil der u-Komponente stromauf, an einem Ort x vor 
der Hinterkante. Die fehlende v-Komponente muss durch numerische Simulation einer laminaren Kanaleinlaufströmung bestimmt werden. Für die Hauptströmung oberhalb und die Spülströmung unterhalb der Platte wurde der Vorlauf unabhängig voneinander simuliert.

Am Kanaleintritt wurde als Einströmrandbedingung eine gleichmäßige, von y unabhängige Geschwindigkeit $U_{0}$ vorgegeben. Diese muss gemäß (4.1) [Schlichting (1951)] so gewählt werden, dass die Kernströmung nach $0.9 \mathrm{~m}$ Lauflänge der gewünschten Geschwindigkeit der freien Strömung $\mathrm{U}_{\infty}$ entspricht.

$$
\mathrm{U}_{0}=\mathrm{U}_{\infty} \frac{\mathrm{a}-\delta_{1}}{\mathrm{a}}
$$

mit a: halbe Kanalhöhe und $\delta_{1}$ Verdrängungsdicke, die für die Hauptströmung im Experiment ermittelt wurde.

Aus den Simulationsdaten wurde das u-Profil extrahiert, welches dem gemessenen am ähnlichsten ist. Wie erwartet, ergab sich eine gute Übereinstimmung der Daten nach ca. 0.9 m Lauflänge der Strömung. Die Strömungsgeschwindigkeit erreicht ihr Maximum außerhalb der Grenzschicht und verringert sich geringfügig zur Kanalmitte hin. Dies ist mit der Produktion eines kleinen Anteils positiver Wirbeligkeit verbunden, wie auch Abbildung 4.3 zeigt. In Abbildung 4.2 sind das numerisch und das experimentell gewonnene Profil für $\mathrm{U}_{\infty}=4.7^{*} 10^{-2} \mathrm{~m} / \mathrm{s}$ zum Vergleich dargestellt.

Für die Spülströmung unterhalb der Splitterplatte lagen zur Definition der Einströmbedingung nur Daten innerhalb der Grenzschicht vor. Da die Geometrie unterhalb der Splitterplatte von der eines Kanals deutlich abweicht, ist die Nachbildung des Strömungsprofils hier nicht so exakt möglich, wie im Bereich der Hauptströmung. Analog wurde hier das Profil einer Kanalströmung 0.9 m stromab des Einströmrandes, an dem ca. 10\% der zugehörigen Hauptströmungsgeschwindigkeit vorgegeben wurden, verwendet. Für die Ausbildung der Scherschicht ist die Hauptströmung dominierend, so dass eventuelle Abweichung des numerisch verwendeten von dem real vorherrschenden Profil der Spülströmung vernachlässigt werden können. 


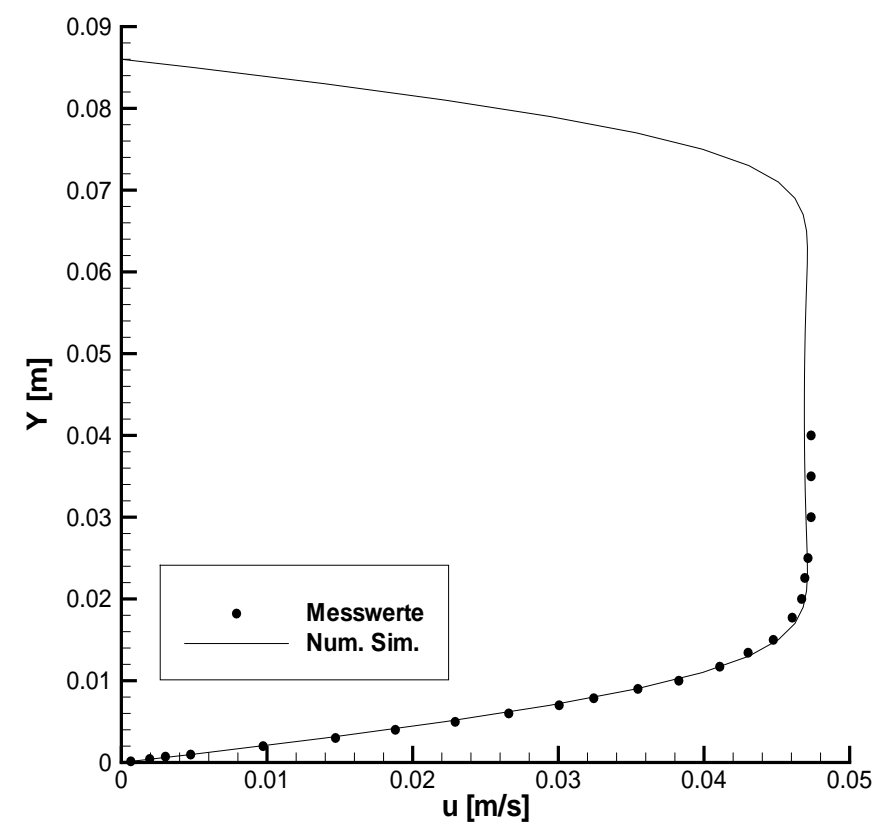

Abbildung 4.2: Vergleich des Einströmprofils, gewonnen aus der numerischen Simulation eines Kanals, mit den Messwerten der Strömungsgeschwindigkeit im Wasserkanal aus [Graf (1998)]

\subsubsection{Eigenschaften der Gleichströmung}

Das Geschwindigkeitsfeld und die Wirbeligkeitsverteilung der Gleichströmung ist in Abbildung 4.3 dargestellt. Aus den Vektoren ist die deutlich höhere Geschwindigkeit der Hauptströmung gegenüber der Spülströmung unterhalb der Platte und deren Grenzschichtprofil erkennbar. Die Wirbeligkeitsverteilung weist ein Maximum entlang der oberen Kanalwand und ein Minimum (Extremum negativer Wirbeligkeit) entlang der Splitterplatte und dann in der Scherschicht auf. An der unteren Kanalwand ist in einem kleinen Bereich stromab der Hinterkante positive Wirbeligkeit erkennbar, die auf eine Strömungsablösung hinweist.

Um die Relaxation einer Scherschicht zu untersuchen, wurden fünf Gleichströmungsszenarien simuliert. Diese unterscheiden sich in der Hauptströmungsgeschwindigkeit von entweder ca. $4.7 * 10^{-2} \mathrm{~m} / \mathrm{s}$ oder ca. $15^{*} 10^{-2} \mathrm{~m} / \mathrm{s}$ in Anlehnung an das Experiment und in der Viskosität des Mediums. Um eine Vergleichbarkeit der numerischen mit den experimentellen Daten zu ermöglichen, wurde die Viskosität $v=1 * 10^{-6} \mathrm{~m}^{2} / \mathrm{s}$ von Wasser bei $20^{\circ} \mathrm{C}$ und deren Vielfache $v=5^{*} 10^{-6} \mathrm{~m}^{2} / \mathrm{s}, \quad v=1^{*} 10^{-5} \mathrm{~m}^{2} / \mathrm{s}$ gewählt. Je größer die Viskosität ist, um so schneller wächst die Grenzschicht. Dies führt zu einer Verdrängung des Fluids aus dem wandnahen Bereich und damit zu einer größeren Impuls- 


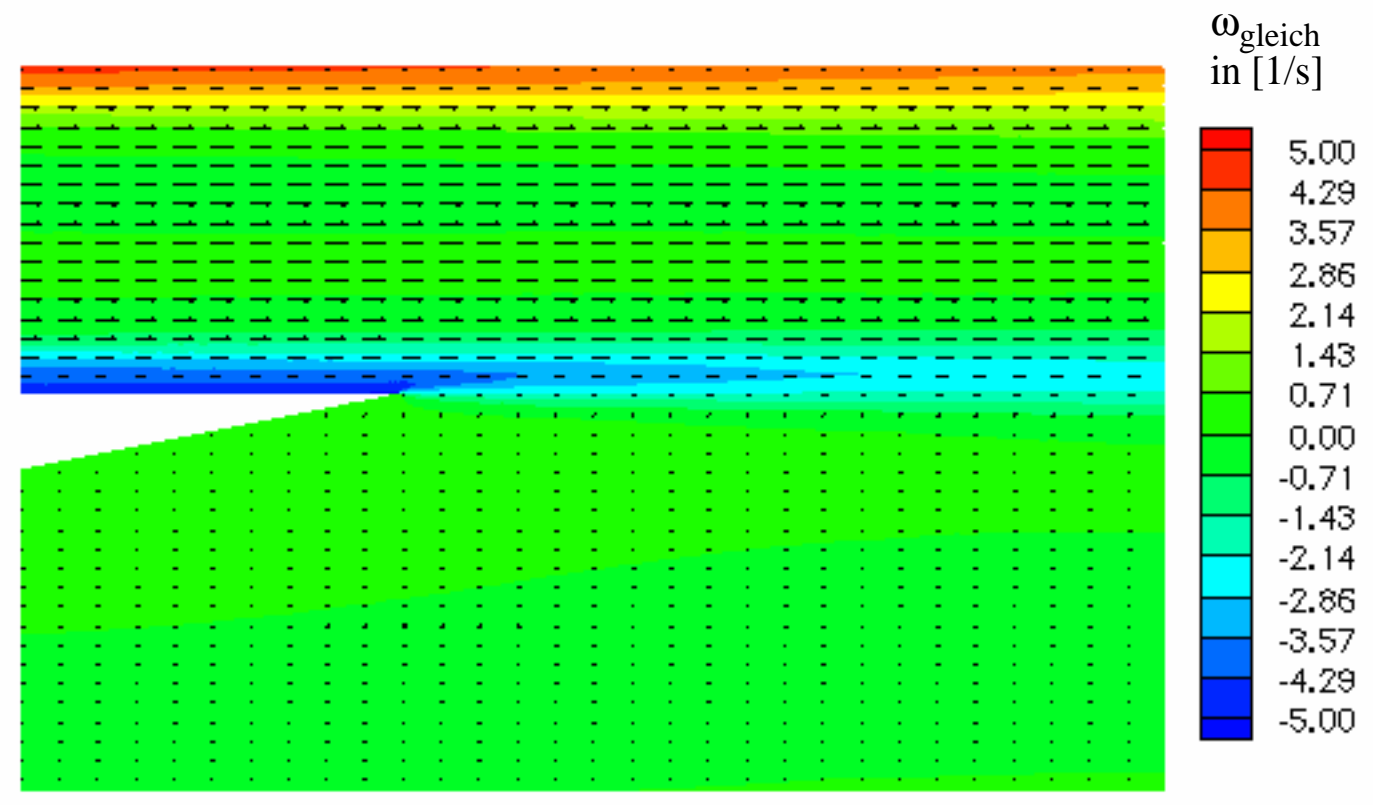

Abbildung 4.3: Geschwindigkeitsvektoren und Wirbeligkeitsverteilung der stationären Gleichströmung in einem Bereich von $0.1 \mathrm{~m}$ stromauf bis $0.2 \mathrm{~m}$ stromab der Hinterkante über die gesamte Kanalhöhe von ca. $0.19 \mathrm{~m}$

verlustdicke $\Theta$, ein für die Dynamik der Scherschicht relevanter Parameter, und zu einer Beschleunigung der Hauptströmung. Beides wurde $0.01 \mathrm{~m}$ stromauf der Hinterkante bestimmt. Ein Vergleich der Daten aus Tabelle 4.1 zeigt dies deutlich.

Tabelle 4.1: Viskosität, Hauptströmungsgeschwindigkeit und resultierende Impulsverlustdicke

\begin{tabular}{|l|l|l|l|}
\hline$v$ in $\left[\mathrm{m}^{2} / \mathrm{s}\right]$ & $\mathrm{U}_{\infty}$ in $[\mathrm{m} / \mathrm{s}]$ & $\Theta$ in $[\mathrm{m}]$ & $\operatorname{Re}_{\Theta}$ \\
\hline $1 * 10^{-6}$ & $4.749 * 10^{-2}$ & $2.59 * 10^{-3}$ & 123 \\
\hline $5 * 10^{-6}$ & $4.903 * 10^{-2}$ & $3.05 * 10^{-3}$ & 30.0 \\
\hline $1 * 10^{-5}$ & $5.056 * 10^{-2}$ & $3.48 * 10^{-3}$ & 17.6 \\
\hline $5 * 10^{-6}$ & $15.46^{*} 10^{-2}$ & $1.87 * 10^{-3}$ & 57.8 \\
\hline $1 * 10^{-5}$ & $15.76^{*} 10^{-2}$ & $2.16^{*} 10^{-3}$ & 34.0 \\
\hline
\end{tabular}

Weitere Informationen bieten die Momente der Wirbeligkeitsverteilung. In den folgenden Abbildungen sind das 0te, 1te und 2te Moment der Wirbeligkeitsverteilung einer Strömung mit ca. $4.7 * 10^{-2} \mathrm{~m} / \mathrm{s}$ Geschwindigkeit und verschieden viskosen Medien dargestellt. 


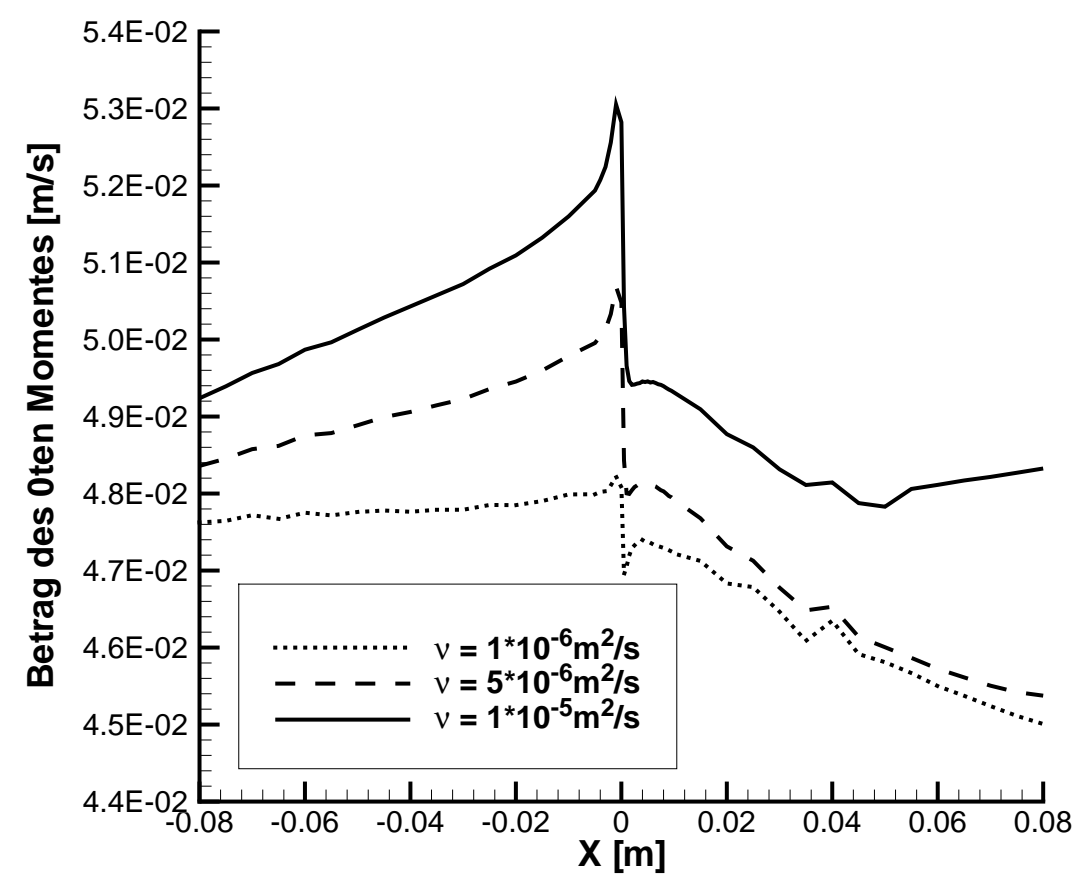

Abbildung 4.4: Otes Moment (Gesamtwirbeligkeit) der Wirbeligkeitsverteilung für drei verschiedene Viskositäten

Abbildung 4.4 zeigt den Betrag des 0ten Momentes, ein Maß für die Gesamtwirbeligkeit, in Abhängigkeit von x. Die mit zunehmender Zähigkeit größere Beschleunigung der Hauptströmung und damit vermehrte Produktion von Wirbeligkeit über der Platte zeigt sich im Anwachsen des Oten Momentes im Bereich negativer x-Koordinaten. Die dann folgende lokal begrenzte Unstetigkeit des Oten Momentes im Bereich der Hinterkante resultiert vom Zusammentreffen der Grenzschichten von Haupt- und Nebenströmung und konnte von [Graf (1998) und Neuwald (1990)] im Experiment ebenfalls beobachtet werden. Das Absinken der Gesamtwirbeligkeit weiter stromab der Kante zeigen auch die Messwerte von [Neuwald (1990)].

Das 1te Moment, dargestellt in Abbildung 4.5, gibt die Lage des Schwerpunktes der Wirbeligkeitsverteilung in Abhängigkeit von $\mathrm{x}$ an. Das Absinken des Verteilungsschwerpunktes hinter der Kante entspricht der Verlagerung der freien Scherströmung in den Bereich der Spülströmung hinein, wie auch im Experiment u.a. von [Neuwald (1990)] beobachtet wurde. 


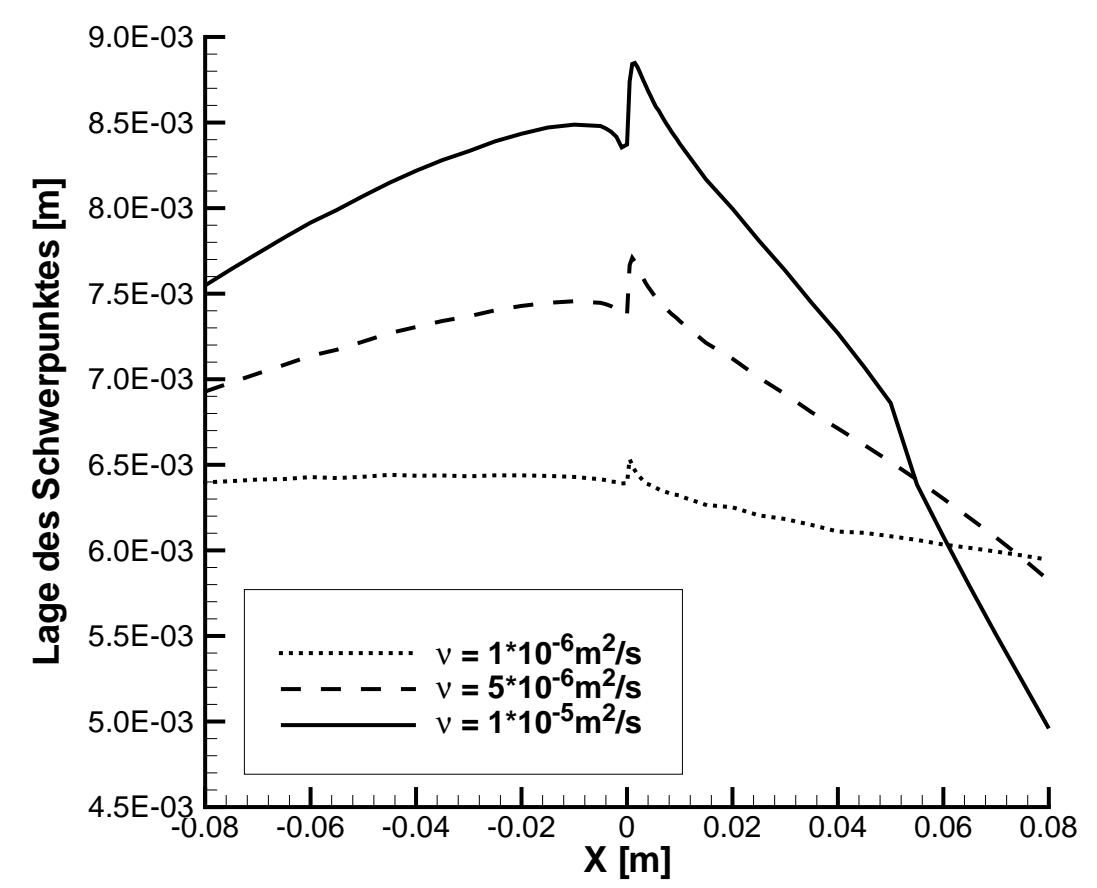

Abbildung 4.5: Lage des Schwerpunktes der Wirbeligkeitsverteilung für drei verschiedene Viskositäten

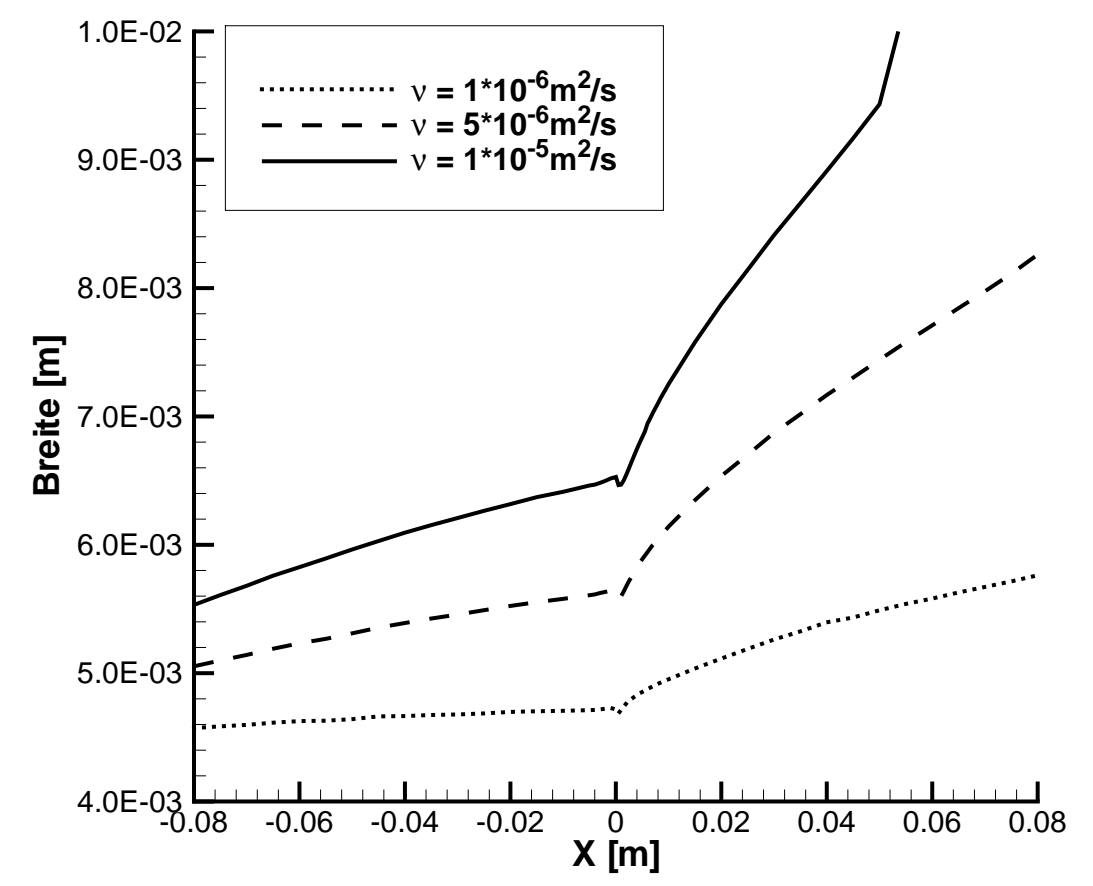

Abbildung 4.6: Breite der Wirbeligkeitsverteilung für drei verschiedene Viskositäten 
Die Scherschichtdicke, d.h. das 2te Moment (Abbildung 4.6), wächst besonders in den Medien mit hoher Viskosität stark an. Über der Platte ist dies nur in positive y-Richtung in die Hauptströmung hinein möglich. Das Wachstum ist daher dort geringer als in der freien Scherschicht, welche sich beidseitig, auch in die Nebenströmung hinein ausdehnen kann.

\section{2 Überlagertes Strömungsfeld}

Während sich die Gleichströmung aufgrund stationärer Randbedingungen entwickelt, kann das überlagerte Strömungsfeld nur durch instationäre Randbedingungen erzeugt werden. Zum Zeitpunkt $t=0$ wird eine Potenzialströmung überlagert. Die hierzu vorgegebene zusätzliche Geschwindigkeitsverteilung am Einströmrand muss auch eine geeignete zeitabhängige Wand-Anschlußbedingung enthalten, die die Diffusion von Wirbeligkeit in die Strömung hinein nachbildet; dies ist in Abschnitt 4.2.1 dargestellt. Die Charakteristika der sich nach diesen Randbedingungen entwickelnden Anregung sind in Abschnitt 4.2.2 beschrieben.

\subsubsection{Instationäre Einströmrandbedingung}

Die Auslenkung der Scherschicht erfolgt durch einen Druckgradientenimpuls, der zur Überlagerung eines Potenzialfeldes zum Zeitpunkt $t=0$, wie in Abbildung 4.7 dargestellt, führt. Diese Form der Anregung wird erreicht, indem nicht der Druckgradientenimpuls direkt vorgegeben wird, sondern durch Definition von instationären Einströmrandbedingungen, aus denen das überlagerte Strömungsfeld resultiert. Dies geschieht durch die Addition eines Kastenprofils zur Hauptströmung und Subtraktion desselben Profils von der Nebenströmung. Da die Strömung nicht reibungsfrei ist und sich somit das Fluid unmittelbar an festen Wänden in Ruhe befindet, muss eine geeignete Anschlussbedingung verwendet werden.

Die angeregte Strömung bewegt sich relativ zur festen Wand mit einer Geschwindigkeit $\mathrm{U}_{\Delta}$. Die zeitliche Entwicklung der Grenzschicht der bewegten Strömung an der ruhenden, ebenen Wand entspricht der einer ruhenden Strömung, nachdem die Wand plötzlich aus der Ruhe heraus, zur Zeit $\mathrm{t}=0$, in ihrer Ebene, mit konstanter Geschwindigkeit $\mathrm{U}_{\Delta}$ in Bewegung gesetzt wird. Hierfür gilt nach [Schlichting (1951)]: 


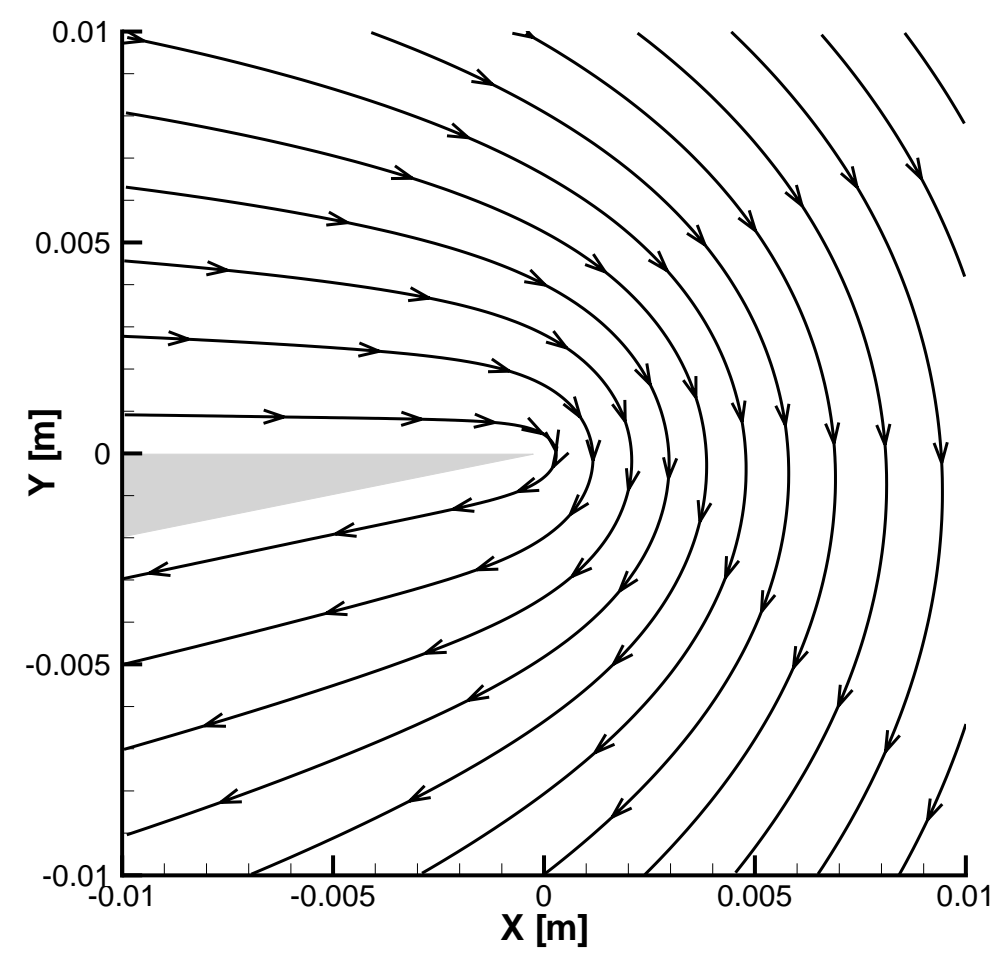

Abbildung 4.7: Angeregtes Potentialfeld nach Veränderung der Einströmrandbedingungen

$$
\frac{\mathrm{u}}{\mathrm{U}_{\Delta}}=\mathrm{f}(\xi)=1-\frac{2}{\sqrt{\pi}} \int_{0}^{\xi} \mathrm{e}^{-\xi^{2} \mathrm{~d} \eta}
$$

Die dimensionslose Variable $\xi$ ist definiert als:

$$
\xi=\frac{\mathrm{y}}{2 \sqrt{v \mathrm{t}}}
$$

Die Abhängigkeit der Geschwindigkeitsverteilung (4.2) von $\xi$ zeigt die Ähnlichkeit der Profile zu verschiedenen Zeitpunkten. Im Fall der überlagerten Strömung ist also deren Geschwindigkeit $U_{\Delta}$ je nach Einfluss der Wand gemäß (4.2) zu verringern. Abbildung 4.8 zeigt die Geschwindigkeitsverteilung in der Nähe der bewegten Wand.

\subsubsection{Charakterisierung der Anregung}

Die strömungsmechanischen Größen innerhalb des Simulationsgebietes entwickeln sich ausschließlich aus den Randbedingungen nach der numerischen Lösung der implementierten elementaren Gleichungen. Da für den zu untersuchenden Bereich um 


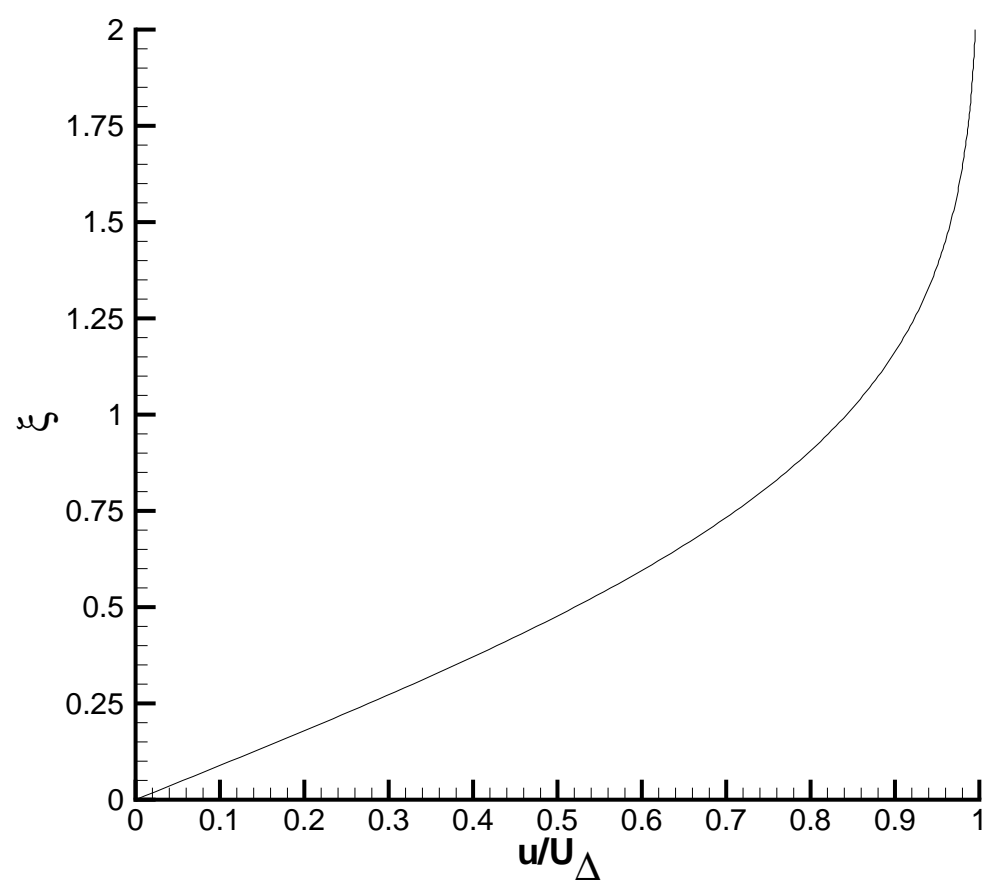

Abbildung 4.8: Geschwindigkeitsverteilung in der Nähe der bewegten Wand

die Hinterkante herum lokal keine direkten Vorgaben gemacht wurden, ist zu prüfen, ob die Randbedingungen nach Lösen von Impuls- und Poisson-Gleichungen in geeigneter Weise wirken:

1) Die vorgegeben Geschwindigkeiten am Einströmrand führen wie erwünscht zur Überlagerung eines Feldes, welches dem gleicht, das durch einen Druckgradientenimpuls entstanden ist. Dies zeigen die Werte des Druckgradienten in Strömungsrichtung, ermittelt an einem Monitorpunkt in der Hauptströmung, dargestellt in Abbildung 4.9. Der Impuls ist genau einen Zeitschritt lang und entspricht somit einer sehr genauen numerische Näherung eines Delta-Druckgradientenimpulses.

2) Das so angeregte Potenzialfeld führt zu einer punktförmigen Erzeugung von Wirbeligkeit an der Hinterkante, wie in Abbildung 4.10 gezeigt. Die konzentrische Verteilung der Wirbeligkeit ist besonders für das Modell der instationären Strömungsablösung bedeutend. Die Güte des Feldes kann anhand des Oten Momentes der Wirbeligkeit beurteilt werden, dieses ist in Abbildung 4.11 dargestellt. Die Integrationsgrenzen erstrecken sich über die Gebiete der Hauptströmung und der freien Scherschicht. In der zweidimensionalen Potenzialströmung entsteht Wirbeligkeit nur an festen Oberflächen, hier über der Platte, im Bereich der negativen x-Koordinate, und 


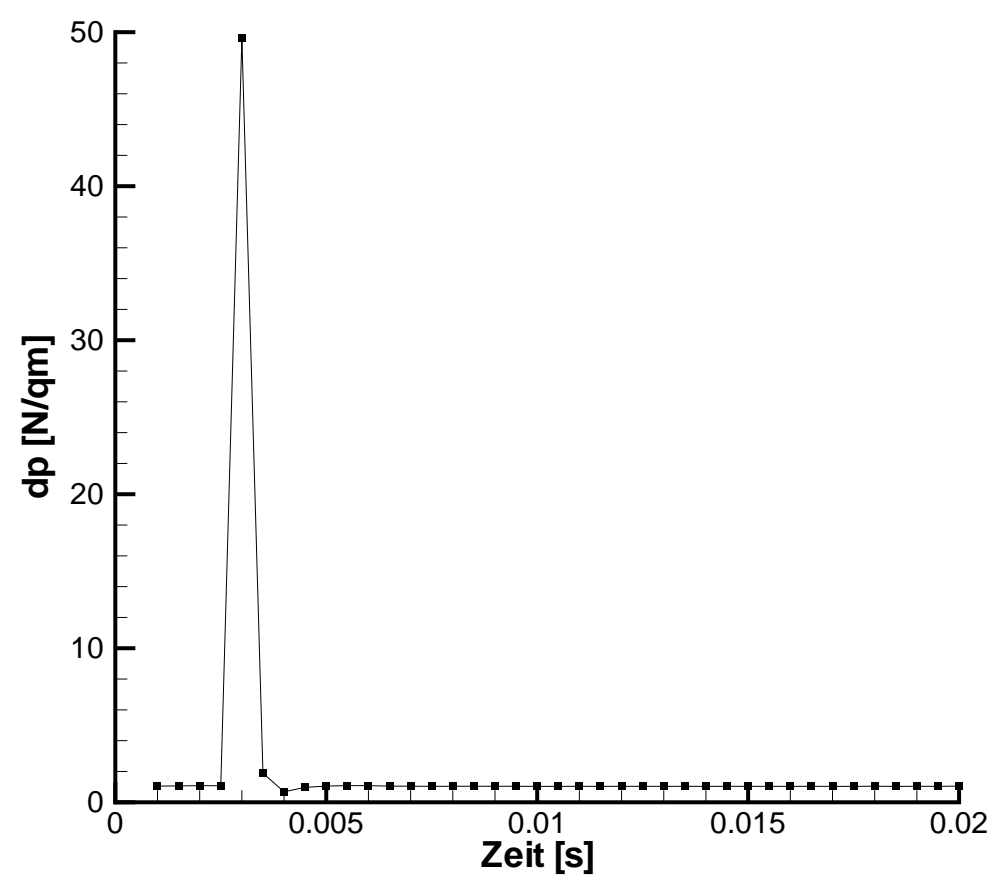

Abbildung 4.9: Druckgradient in Strömungsrichtung

direkt an der Hinterkante, aufgrund deren Umströmung. Nach der Theorie der Potenzialströmung (s. Abschnitt 2.1 auf Seite 8) ist diese Umströmung unendlich schnell, nicht aber im realen, reibungsbehafteten Fluid. Im 0ten Moment der Wirbeligkeitsverteilung äußert sich dieser Sachverhalt in einem scharf begrenzenten Extremum an der Stelle der Hinterkante.

Die instationären Randbedingungen erfüllen somit alle relevanten Kriterien, d.h. die Anregung durch Änderung der Einströmrandbedingungen entspricht der eines Druckgradientenimpulses. Das überlagerte Strömungsfeld läßt sich im Wesentlichen nach der Potenzialtheorie beschreiben, ist also im Innern wirbelfrei. Es verfügt über geeignete Wand-Anschlußbedingungen, die unter Berücksichtigung der Viskosität die Diffusion von Wirbeligkeit in die Strömung hinein nachbilden.

\subsection{Stationärer Endzustand}

Die zeitabhängige Wechselwirkung der stationären Gleichströmung (Abschnitt 4.1) und deren Anregung (Abschnitt 4.2) ist Gegenstand der folgenden Kapitel. In diesem Abschnitt werden die Eigenschaften des Strömungsfeldes eines stationären Endzustands beschrieben, der sich nach vollständig abgeschlossener Relaxation einstellt. 


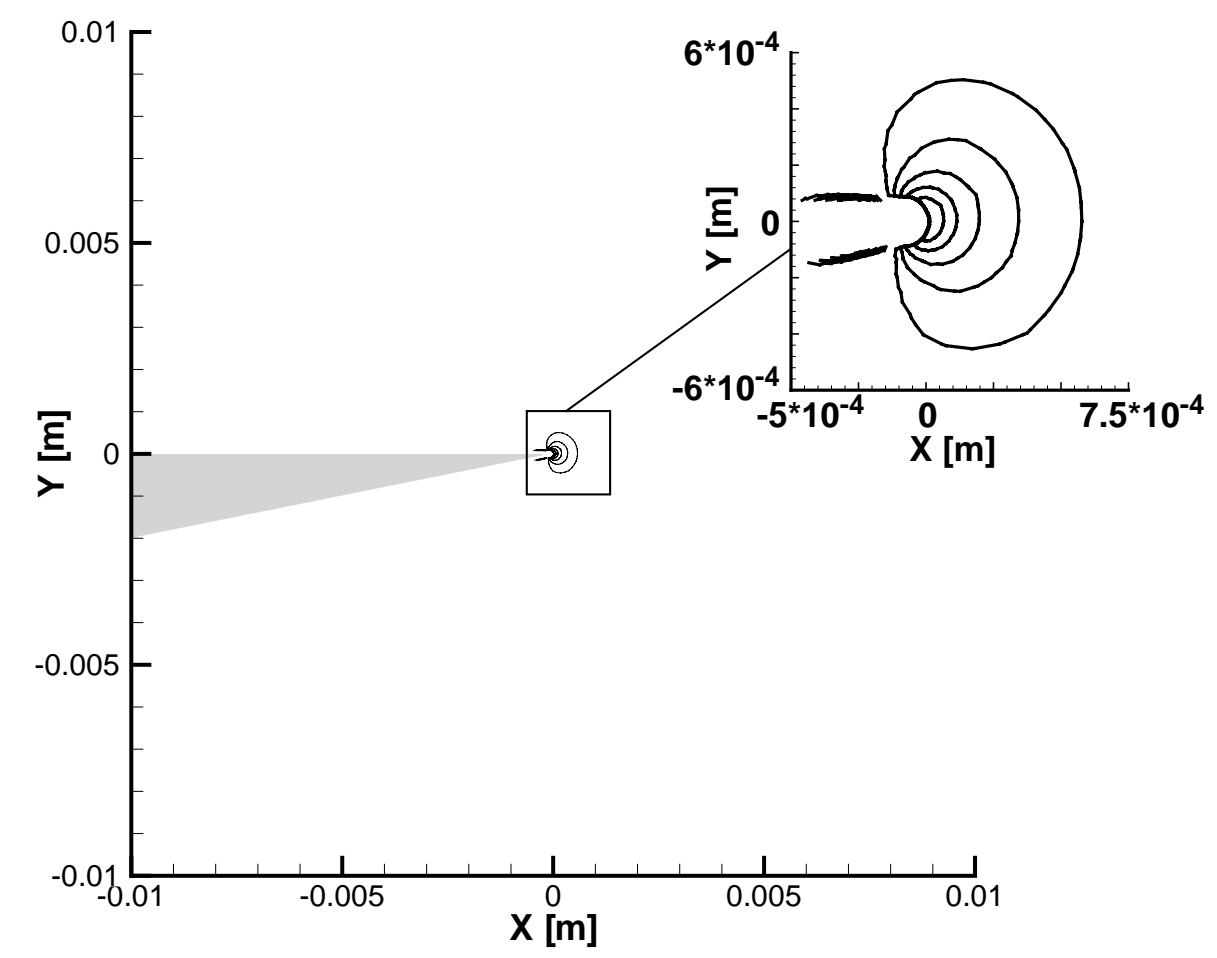

Abbildung 4.10: Punktförmige Erzeugung von Wirbeligkeit an der Hinterkante. Die äußere Isolinie stellen einen Bereich dar, in dem die Wirbelstärke von ihrem Maximum auf 1/e abgefallen ist.

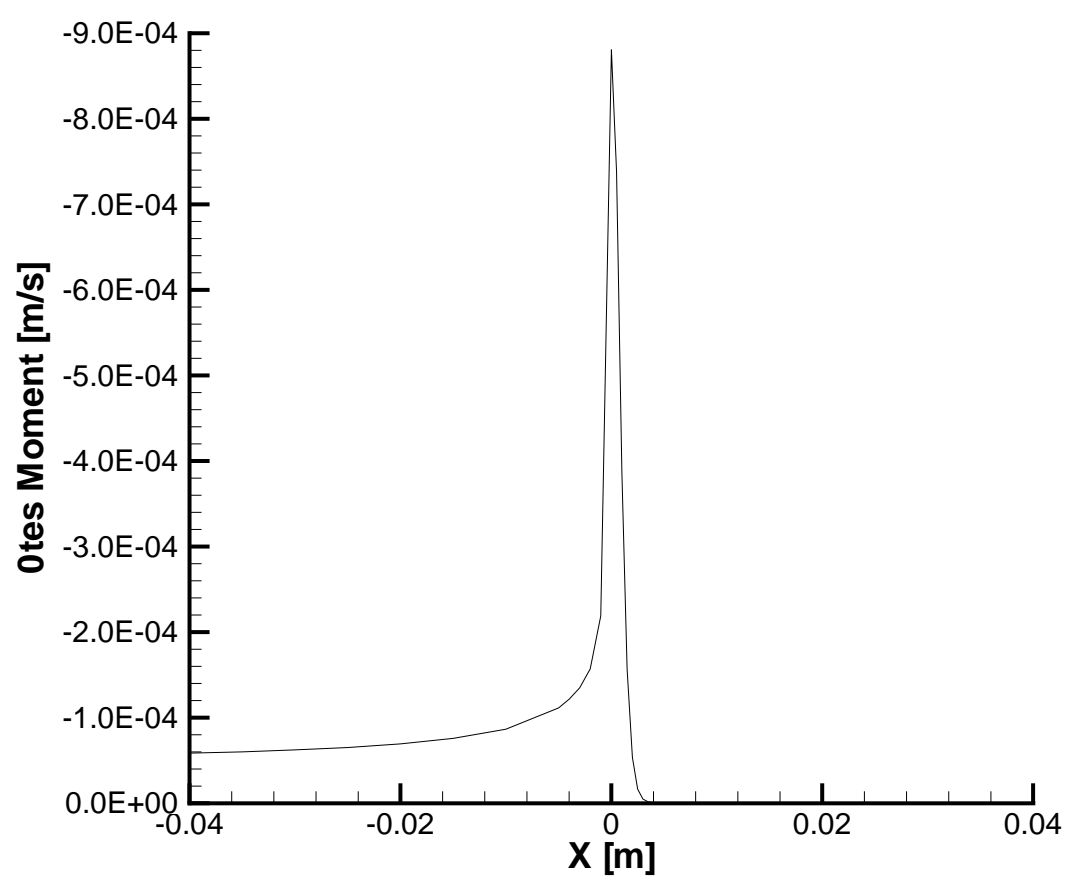

Abbildung 4.11: Das 0te Moment zeigt ein lokal begrenztes Extremum der Wirbeligkeit unmittelbar nach Anregung mit einer Amplitude von $\mathrm{U}_{\Delta}=5^{*} 10^{-5} \mathrm{~m} / \mathrm{s}\left(0.1 \%\right.$ von $\left.\mathrm{U}_{\infty}\right)$. 
Ist die Dynamik der Wechselwirkung von Gleichströmung und überlagertem Potenzialfeld abgeklungen ( $\mathrm{t} \gg \mathrm{T}_{\text {relax }}$ ), so entwickelt sich das Strömungsfeld ausschließlich nach den (stationären) Randbedingungen. Diese setzen sich zusammen aus den Einströmrandbedingungen der Gleichströmung und der Anregung zum entsprechenden Zeitpunkt t. Nach einer ausreichend langen Zeit sind diese zeitabhängigen Randbedingungen nahezu konstant (s. Gleichung (4.3)). Eine Simulation mit solchen Vorgaben liefert somit den stationären Endzustand nach Anregung mit einem DruckgradientenImpuls und vollständiger Relaxation der Scherschicht. Der numerische Aufwand dieser Vorgehensweise ist relativ gering. Eine auskonvergierte stationäre Lösung wird nach deutlich weniger Iterationen erreicht, als zur Simulation des gesamten Relaxationsprozesses, bzw. darüber hinaus, mit der notwendigen zeitlichen Diskretisierung notwendig sind.

Die Relaxation der Scherschicht wird im Modell durch die Umverteilung von Wirbeligkeit durch Konvektion und Diffusion beschrieben. Abbildung 4.12 zeigt, dass die unmittelbar nach der Anregung an der Kante konzentrierte Wechselwirbeligkeit (Wirbeligkeit der angeregten Strömung nach Abzug der Wirbeligkeitsverteilung der stationären Gleichströmung) tangential abschwimmt. Das Maximum der Differenz hat sich auf die gesamte Scherschicht ausgedehnt. Die folgenden Ergebnisse stammen aus der Simulation einer Strömung mit ca. $5^{*} 10^{-2} \mathrm{~m} / \mathrm{s}$ Hauptströmungsgeschwindigkeit und einer Viskosität von $1 * 10^{-5} \mathrm{~m}^{2} / \mathrm{s}$.

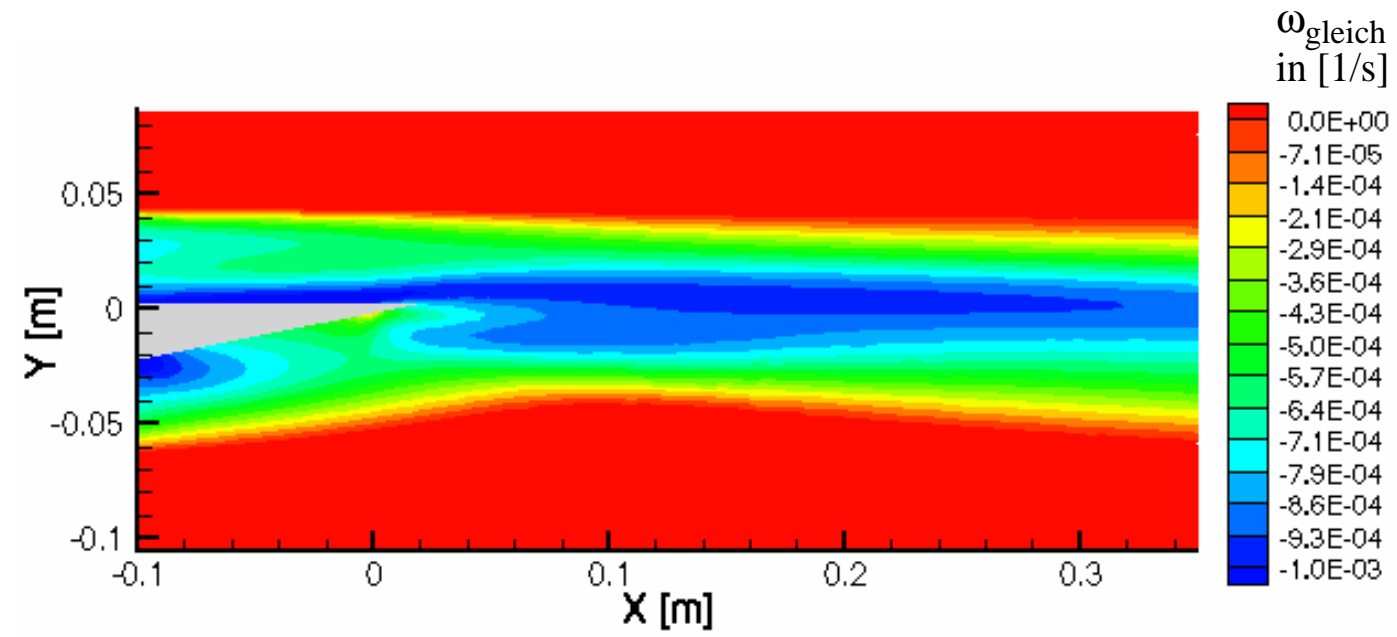

Abbildung 4.12: Wechselwirbeligkeit eines stationären Endzustandes $100 \mathrm{~s}$ nach der Anregung 
Der dargestellte Wertebereich der Wirbeligkeitsverteilung ist um mehrere Größenordnungen kleiner als in Abbildung 4.3. Um den Beitrag der überlagerten Strömung zu quantifizieren, ist in Abbildung 4.13 die relative Änderung der Momente dargestellt. Dabei ist die Differenz der Momente des Endzustandes mit dem Betrag der Momente der stationären Gleichströmung normiert worden. Die leicht erhöhte Strömungsgeschwindigkeit führt zur Produktion von etwas mehr Wirbeligkeit, wie das 0te Moment zeigt. Aufgrund der hohen Viskosität und der vergangenen, langen Zeit nach der Anregung ist die wirksame Geschwindigkeit des überlagerten Feldes auf 0.05\% zurückgegangen. Der Wert des Oten Momentes ist konsistent mit dem resultierenden Strömungsprofil, wie ein Vergleich gezeigt hat. Der Schwerpunkt der Wirbeligkeitsverteilung des Endzustandes liegt zunächst höher, sinkt dann aber im weiteren Verlauf stromab der Kante stärker ab als im Ausgangszustand. Dies zeigt der Vorzeichenwechsel des 1ten Momentes. Die Ausdehnung der Scherschicht ist ebenfalls gewachsen, wie dem 2ten Moment zu entnehmen ist. Alle Effekte bewegen sich im Bereich von 0.2\%, was etwa dem Verhältnis der Strömungsgeschwindigkeiten von Anregung zu Grundströmung entspricht.

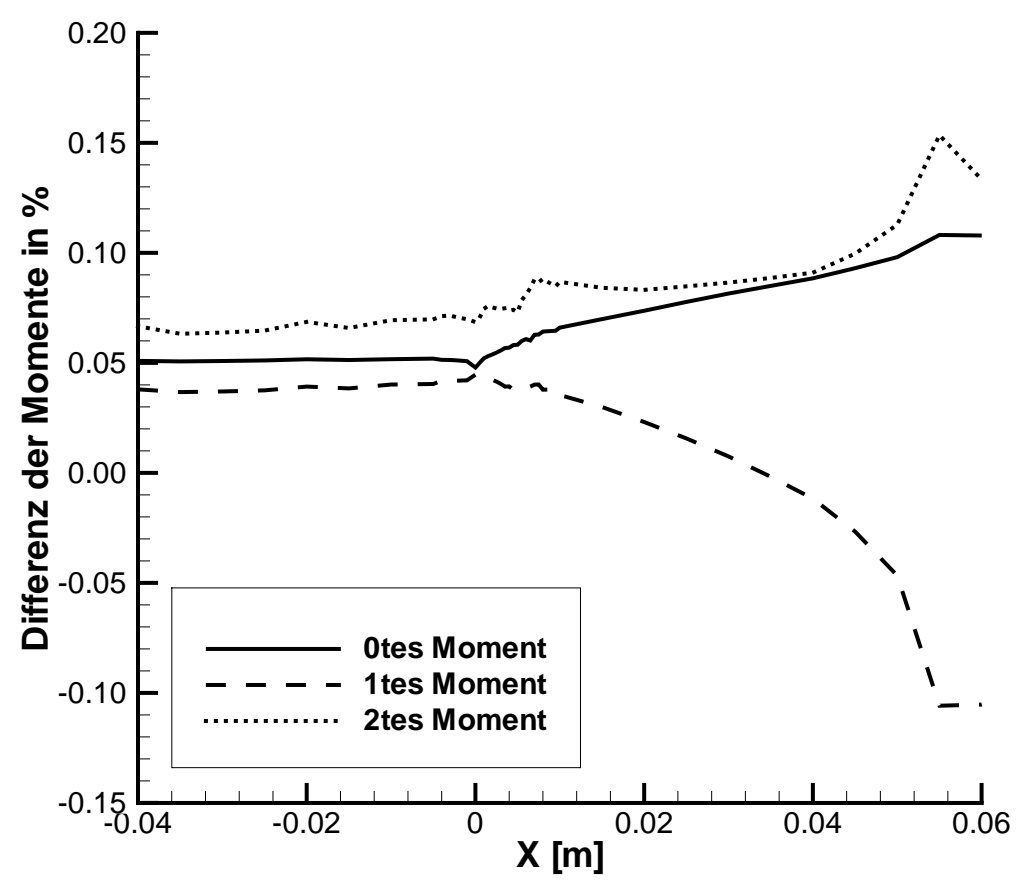

Abbildung 4.13:Differenz der Momente des Endzustandes normiert mit dem Betrag der Momente der stationären Gleichströmung 
Die Stromlinien des überlagerten Feldes (vgl. Abbildung 4.7) sind im stationären Endzustand parallel zur Hauptströmungsrichtung, d.h. die Umspülung der Kante wurde vollständig abgebaut, wie Abbildung 4.14 zeigt. Das resultierende Strömungsfeld erfüllt die reine Kutta-Bedingung.

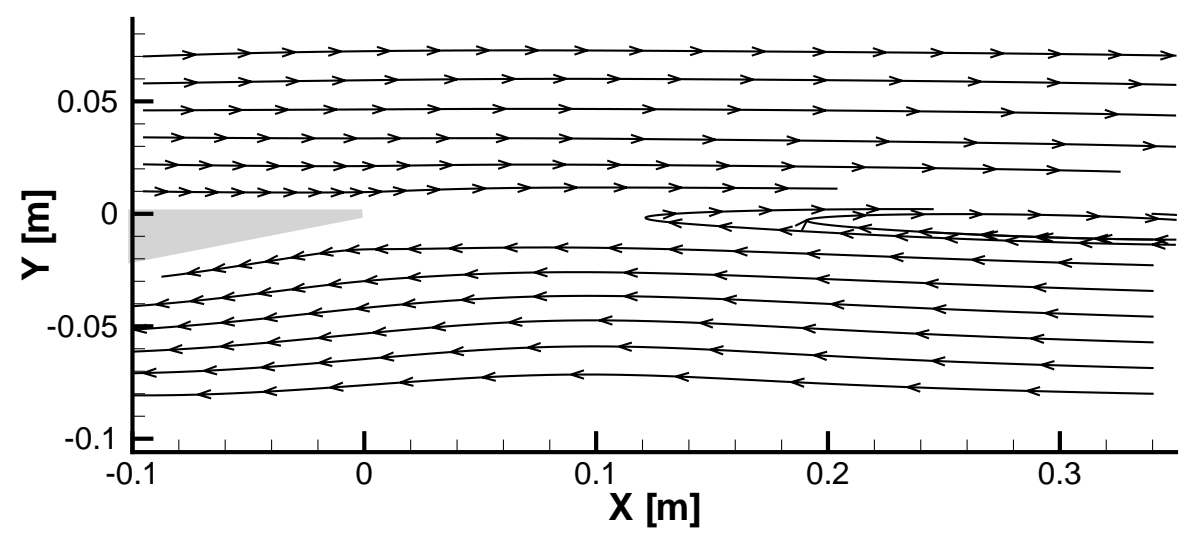

Abbildung 4.14:Stromlinien der Differenz-Geschwindigkeit $100 \mathrm{~s}$ nach der Anregung 


\section{Scherschichtauslenkung}

Zur Charakterisierung der instationären Strömungsablösung wird vielfach die räumliche und zeitliche Entwicklung der Scherschichtauslenkung untersucht, wie z.B. in [Orszag \& Crow (1970)] und [Daniels (1978)]. Die instantane Überlagerung der Potenzialströmung entspricht einer Störung, die sich mit der Zeit in Strömungsrichtung fortsetzt und die Scherschicht auslenkt. Das Maß dieser Auslenkung kann unter Verwendung des 1ten Momentes der Wirbeligkeitsverteilung quantifiziert werden. Dazu wird ausschließlich die Veränderung der Gesamtverteilung berücksichtigt, also nicht der Anteil der stationären Gleichströmung.

In Abschnitt 5.1 wird zunächst eine Normierung des 1ten Momentes eingeführt, die im Folgenden verwendet wird. Unterschiedliche Eigenschaften der Grundströmung und der Anregung führen zu einer unterschiedlich starken Auslenkung der Scherschicht. Um diese dennoch vergleichbar zu machen und auf die ausschlaggebenden Prozesse zurückzuführen, ist eine sinnvolle physikalische Normierung unumgänglich.

Die Scherschichtauslenkung ist sowohl vom Ort x stromab der Hinterkante, als auch von der Zeit t nach der Anregung abhängig. Diese räumliche und zeitliche Entwicklung ist nicht einheitlich. Die Auslenkung wird von verschiedenen Effekten beeinflusst, wie in Abschnitt 5.2 erläutert wird.

Das Erreichen der maximalen Auslenkung der Wirbeligkeitsschicht während des Auslenkungsprozesses wird in Abschnitt 5.3 quantitativ beschrieben. Vom numerischen Standpunkt aus gesehen ist dieser Prozess wichtig, da die hierzu benötigte Zeit die kürzeste numerisch aufzulösende Zeit ist. Besonders während des Auslenkungsprozesses treten hohe Gradienten auf. Es werden daher hohe Anforderungen an die zeitliche Diskretisierung gestellt, die in einer Studie bestimmt wurden. Dazu wurde die Abhängigkeit der Zeit, bis unmittelbar hinter der Kante das Maximum des 1ten Momentes erreicht ist untersucht. Es zeigte sich der Einfluss des numerischen Zeitschritts, wenn dieser deutlich größer als $0.001 \mathrm{~s}$ gewählt wurde. Für die simulierte Strömung $\left(\mathrm{U}_{\infty}=4.7 * 10^{-2} \mathrm{~m} / \mathrm{s}\right.$ bei $\left.\mathrm{v}=1 * 10^{-6} \mathrm{~m}^{2} / \mathrm{s}\right)$ entspricht dies $\mathrm{t} / \mathrm{T}_{\mathrm{konv}}=0.018$.

Der Relaxationsprozess (Abschnitt 5.4) beschreibt die Rückkehr der Scherschicht in ihre Ruhelage. Er ist von besonderer praktischer Bedeutung, da die Relaxationszeit eine Abschätzung der Gültigkeit der quasistationären Kutta-Bedingung zuläßt. Damit 
verbunden ist der zu simulierende Zeitraum, als längste Zeiteinheit. Zwischen diesem und dem notwendigen Zeitschritt lagen für die durchgeführten Berechnungen vier Größenordnungen.

\subsection{Normierung des 1ten Momentes}

Die Auslenkung der Scherschicht soll auch für Strömungen mit unterschiedlicher Zähigkeit und verschiedenen Hauptströmungsgeschwindigkeiten vergleichbar sein. Dazu muss das Ergebnis geeignet normiert werden. Das 1te Moment $\mathrm{Y}_{\mathrm{mom}}$, bzw. dessen Änderung, als Maß für die Scherschichtauslenkung $\eta$ muss sich daher auf eine charakteristische Länge $\gamma$ beziehen. Diese Normierung sollte sich aus den strömungsphysikalischen Eigenschaften der Scherschicht und deren Anregung ableiten. Nur auf diese Weise können jeweils gleiche physikalische Eigenschaften zum gleichen Resultat führt. Die folgende Vorgehensweise orientiert sich an [Ronneberger, private Mitteilungen].

Die Auslenkung der Scherschicht durch das überlagerte Potenzialfeld ist im Wesentlichen proportional zur v-Komponente der Anregung $\mathrm{v}_{\Delta}$. Für die Umströmung einer Hinterkante gilt für die v-Komponente nach der Potenzialtheorie:

$$
\mathrm{v}_{\Delta}=\frac{\mathrm{A}_{\Delta}}{\sqrt{\mathrm{x}}}
$$

$\mathrm{A}_{\Delta}$ ist die Amplitude des Potenzialfeldes, diese wird nach dem Fluss bestimmt, welcher oberhalb der Platte $(\mathrm{y}>0)$ in das Berechnungsgebiet einströmt und es unterhalb der Splitterplatte $(\mathrm{y}<0)$ wieder verläßt. Dieser Fluss geht auch durch die Ebene der Scherschicht $(\mathrm{y}=0)$. Deren Auslenkung ist somit abhängig von der Anregungsgeschwindigkeit $U_{\Delta}$ und der Höhe der Einströmränder H. Setzt man diese Flüsse gleich, so ergibt sich:

$$
\mathrm{U}_{\Delta} \cdot \mathrm{H}=\int_{0}^{\cdots} \mathrm{v}_{\Delta} \mathrm{dx}=\int_{0} \frac{\mathrm{A}_{\Delta}}{\sqrt{\mathrm{x}}} \mathrm{dx}
$$


Die Abnahme der v-Komponente mit $1 / \sqrt{\mathrm{x}}$ gilt nur bis zu einer abzuschätzenden Obergrenze, danach kommt es zu einem exponentiellen Abfall. Diese Grenze ist von der Ausdehnung der anregenden Strömung abhängig. Im vorliegenden Fall entspricht dies der Kanalhöhe H, zu der die obere Integrationsgrenze abgeschätzt wird:

$$
\mathrm{U}_{\Delta} \cdot \mathrm{H} \sim \int_{0}^{\mathrm{H}} \frac{\mathrm{A}_{\Delta}}{\sqrt{\mathrm{x}}} \mathrm{dx}=2 \mathrm{~A}_{\Delta} \cdot \sqrt{\mathrm{H}}
$$

Damit folgt für $\mathrm{A}_{\Delta}$ :

$$
\mathrm{A}_{\Delta} \sim \frac{1}{2} \mathrm{U}_{\Delta} \sqrt{\mathrm{H}}
$$

Zusammen mit (5.1) ergibt sich $\mathrm{v}_{\Delta}$ zu:

$$
\mathrm{v}_{\Delta} \sim \frac{1}{2} \mathrm{U}_{\Delta} \sqrt{\frac{\mathrm{H}}{\mathrm{x}}}
$$

Die Auslenkung der Scherschicht ist auch von den Eigenschaften der Hauptströmung abhängig, die bisher noch nicht eingegangen sind. Es ist in diesem Zusammenhang sinnvoll, zur Normierung den Versatz $\gamma$ eines Fluidelements zu verwenden, welches eine Strecke der Länge $\Theta$ mit der Geschwindigkeit $U_{\infty}$ zurückgelegt hat. Dies geschieht innerhalb der Konvektionszeit (vgl. Abschnitt 2.3.2 auf Seite 14):

$$
\mathrm{T}_{\mathrm{konv}} \sim \frac{\Theta}{\mathrm{U}_{\infty}}
$$

Die entsprechende Verschiebung des Fluidelements durch Einwirken von $v_{\Delta}$ berechnet sich damit zu:

$$
\int_{0}^{\mathrm{T}_{\text {konv }}} \mathrm{v}_{\Delta} \mathrm{dt} \sim \int_{0}^{\Theta} \frac{\mathrm{v}_{\Delta}}{\mathrm{U}_{\infty}} \mathrm{dx}
$$

Mit (5.5) folgt daraus für die charakteristische Länge $\gamma$ der Scherschichtauslenkung:

$$
\gamma=\frac{\mathrm{U}_{\Delta}}{\mathrm{U}_{\infty}} \sqrt{\mathrm{H} \Theta}
$$


In Tabelle 5.1 ist $\gamma$ für die verschiedenen nachfolgend ausgewerteten Strömungen zusammengestellt. Die normierte Scherschichtauslenkung $\eta(x, t)$ ergibt sich jeweils aus:

$$
\eta(x, t)=\left|Y_{\text {mom }}(x, t)\right| / \gamma
$$

Tabelle 5.1: Normierungsfaktor $\gamma$ der Scherschichtauslenkung für verschiedene Strömungen

\begin{tabular}{|l|l|l|l|l|l|}
\hline $\begin{array}{l}v \\
\text { in }\left[\mathrm{m}^{2} / \mathrm{s}\right]\end{array}$ & $\begin{array}{l}\mathrm{U}_{\infty} \\
\text { in }[\mathrm{m} / \mathrm{s}]\end{array}$ & $\begin{array}{l}\Theta \\
\text { in }[\mathrm{m}]\end{array}$ & $\begin{array}{l}\mathrm{T}_{\mathrm{konv}} \\
\text { in [s] }\end{array}$ & $\begin{array}{l}\mathrm{U}_{\Delta} \\
\text { in }[\mathrm{m} / \mathrm{s}]\end{array}$ & $\begin{array}{l}\gamma \\
\text { in }[\mathrm{m}]\end{array}$ \\
\hline $1 * 10^{-6}$ & $4.749 * 10^{-2}$ & $2.59 * 10^{-3}$ & 0.05458 & $5 * 10^{-5}$ & $1.571 * 10^{-5}$ \\
\hline $5 * 10^{-6}$ & $4.903 * 10^{-2}$ & $3.05 * 10^{-3}$ & 0.06219 & $5 * 10^{-5}$ & $1.652 * 10^{-5}$ \\
\hline $1 * 10^{-5}$ & $5.056^{*} 10^{-2}$ & $3.48 * 10^{-3}$ & 0.06875 & $5 * 10^{-5}$ & $1.711 * 10^{-5}$ \\
\hline $5 * 10^{-6}$ & $15.46^{*} 10^{-2}$ & $1.87 * 10^{-3}$ & 0.01209 & $1.5^{*} 10^{-4}$ & $1.230^{*} 10^{-5}$ \\
\hline $1 * 10^{-5}$ & $15.76^{*} 10^{-2}$ & $2.16^{*} 10^{-3}$ & 0.01371 & $1.5^{*} 10^{-4}$ & $1.297 * 10^{-5}$ \\
\hline
\end{tabular}

\subsection{Zeitliche und räumliche Entwicklung}

Die Anregung der Scherschicht bewirkt eine unterschiedlich starke Auslenkung, die sich mit der Zeit stromab fortsetzt. Abbildung 5.1 zeigt dies in einer Folge von Zeitschritten. Zunächst ist die Wirkung der Anregung auf eine Region unmittelbar hinter der Kante begrenzt, dort ist die Auslenkung maximal (Abbildung 5.1, $\mathrm{t}=0.02 \mathrm{~s}$ ). Mit fortschreitender Zeit schwimmt dieses Maximum stromab und verbreitert sich. Nach ca. $0.2 \mathrm{~s}$ ist ein Überschwingen zu erkennen, welches die Auslenkung lokal verringert. Auch dieses Extremum verbreitert sich nachfolgend, ist jedoch nahezu ortsfest (vgl. Zeitschritte $0.3 \mathrm{~s}, 0.4 \mathrm{~s}$ und $0.5 \mathrm{~s}$ ). Gemäß dem Modell der instationären Strömungsablösung (vgl. Abschnitt 2.3 auf Seite 11) erwartet man für eine hohe Strouhalzahl, bzw. während einer kurze Anregungsdauer, eine Scherschichtauslenkung $\eta \sim x^{-1 / 2} \cdot t$. Dies ist in den Diagrammen für die ersten drei Zeitschritte $(0.02 \mathrm{~s}, 0.1 \mathrm{~s}$ und $0.2 \mathrm{~s})$ in Abbildung 5.1 als gestrichelte Kurve dargestellt. Unmittelbar nach der Anregung kann die Auslenkung sehr gut angepasst werden, mit fortschreitender Zeit hingegen werden die Abweichungen größer. Hier trägt bereits die Konvektion wirbeligkeitsbehafteter 
Fluidelemente zur Auslenkung der Scherschicht bei. Das Strömungsfeld wird aber auch zunehmende von anderen Mechanismen mit einer anderen Zeitskalen bestimmt, so dass eine Beschreibung an Komplexität zunimmt.
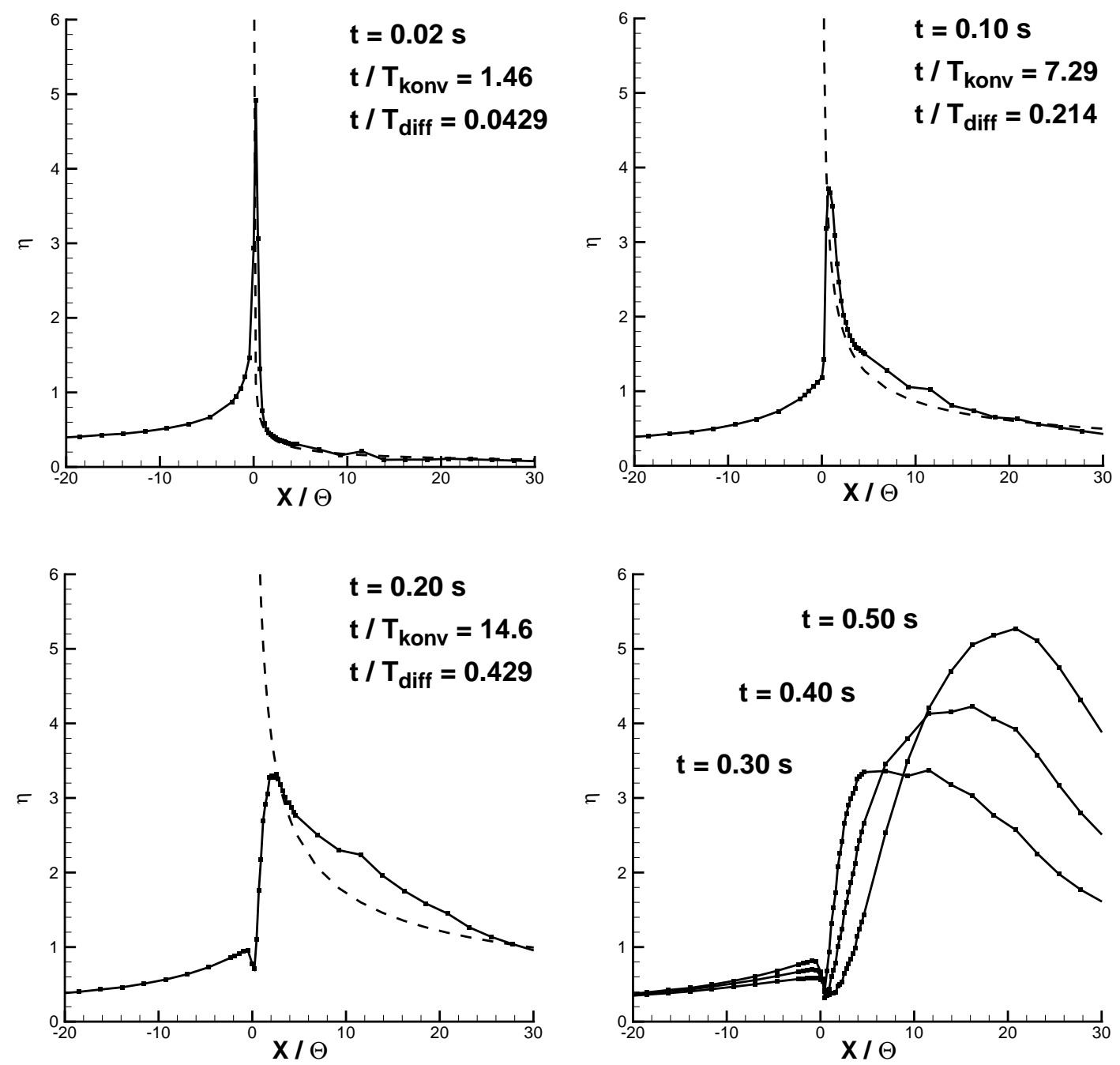

Abbildung 5.1: Folge der normierten Scherschichtauslenkung zu verschiedenen Zeitpunkten $t$ nach der Anregung. Die gestrichelte Kurve in den Diagrammen der Zeitschritte $0.002 \mathrm{~s}, 0.1 \mathrm{~s}$ und $0.2 \mathrm{~s}$ geben die im Text erläuterte Kurvenanpassung wieder. Für die Umrechnung der Zeiten des Diagramms unten rechts gilt: $\quad \mathrm{t}=0.30 \mathrm{~s}: \mathrm{t} / \mathrm{T}_{\text {konv }}=21.9 ; \mathrm{t} / \mathrm{T}_{\text {diff }}=0.643$; $\mathrm{t}=0.40 \mathrm{~s}: \mathrm{t} / \mathrm{T}_{\text {konv }}=29.2 ; \mathrm{t} / \mathrm{T}_{\text {diff }}=0.857$; $\mathrm{t}=0.50 \mathrm{~s}: \mathrm{t} / \mathrm{T}_{\text {konv }}=36.5 ; \mathrm{t} / \mathrm{T}_{\text {diff }}=1.07$;

Nachdem das Maximum des 1ten Momentes zunächst abnimmt (bis ca. $0.2 \mathrm{~s}$ nach der Anregung), ist im späteren Verlauf ein Anwachsen zu beobachten (vgl. Abbildung 5.1, für die Zeitschritte $0.3 \mathrm{~s}, 0.4 \mathrm{~s}$ und $0.5 \mathrm{~s}$ ). Dies zeigt auch der Wertebereich des 1ten Momentes in Abhängigkeit vom Ort x hinter der Kante (Abbildung 5.2). Unmittelbar 
an der Hinterkante (hier $1.0^{*} 10^{-5} \mathrm{~m}$ ) dämpft die Viskosität die Auslenkung der Scherschicht (vgl. Abschnitt 5.3.2), dies kann auch Abbildung 5.3 entnommen werden. Bereits an der folgenden Position $0.5^{*} 10^{-3} \mathrm{~m}(\mathrm{x} / \Theta=0.23)$ hinter der Spitze der Kante hat das 1te Moment sein Maximum erreicht. Stromab verringert sich dann zunächst die Wirkung der Anregung, bis wieder ein Anwachsen der maximalen Auslenkung, nun auch in positive y-Richtung, zu beobachten ist. Dieses Verhalten ist auf zwei unterschiedliche Mechanismen, die eng miteinander verknüpft sind, zurückzuführen. Die anfängliche Abnahme der Auslenkung nach Erreichen des Maximums zeigt, dass die Intensität der Störung stromab nachläßt. Das erneute Anwachsen der Amplitude der Scherschichtbewegung beruht auf der Entstehung einer Kelvin-Helmholtz-Instabilität.

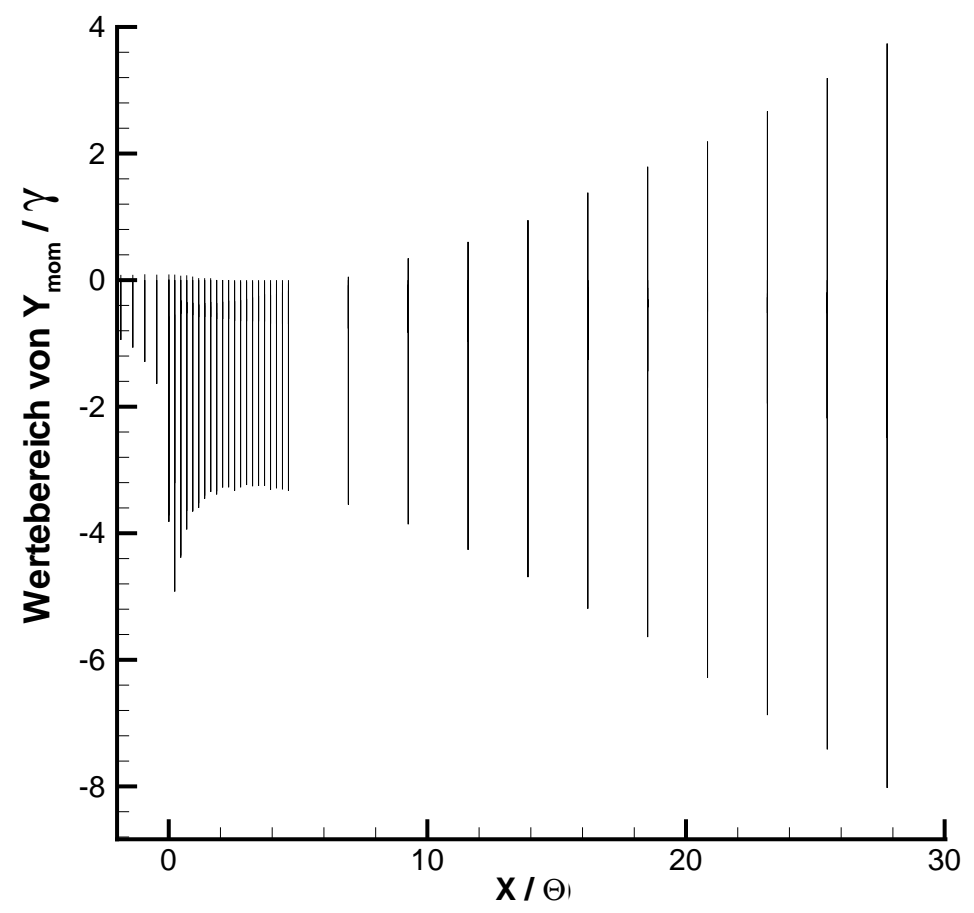

Abbildung 5.2: Amplitude des 1ten Momentes der Wirbeligkeitsverteilung einer angeregten Gleichströmung nach Abzug des stationären Anteils.

Die zeitliche Entwicklung des 1ten Momentes zeigt weitere Charakteristika der beteiligten Prozesse. In Abbildung 5.3 ist dies für Messorte dicht hinter der Kante dargestellt. Diese Region ist durch den ursprünglichen Ablöseprozess dominiert. Das Extremum der Auslenkung der Scherschicht wird nicht überall gleichzeitig erreicht. Mit wachsendem Abstand vergrößert sich auch die Zeit, bis die Störung maximal wirksam ist. Es existiert demnach eine Ausbreitungsgeschwindigkeit der Störung. Mit grö- 
ßerer Entfernung von der von Kante bzw. längerer Wirkungszeit nimmt darüber hinaus die maximale Auslenkung der Scherschicht ab. Eine genaue Untersuchung der Zeit bis die Scherschicht vollständig ausgelenkt wurde, findet sich in Abschnitt 5.3.3.

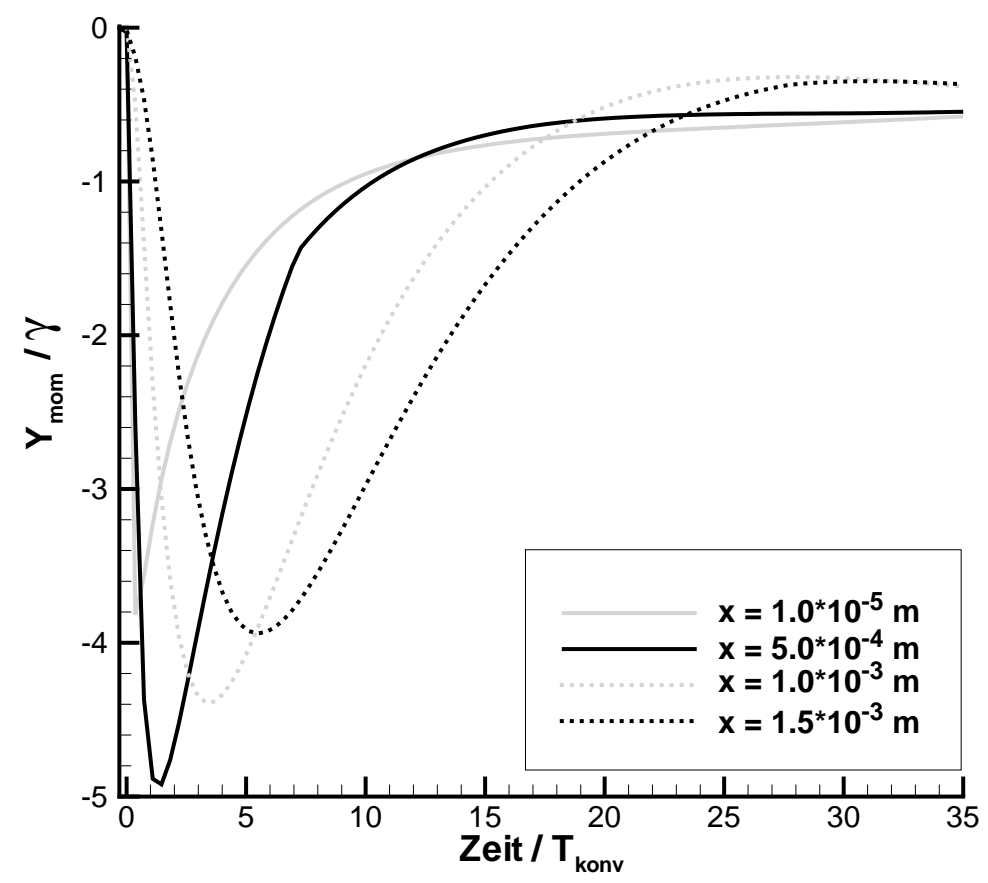

Abbildung 5.3: 1tes Moment der ausgelenkten Scherschicht (ohne Gleichanteil) an unterschiedlichen Messorten $\left(1.0^{*} 10^{-5} \mathrm{~m}, 5.0^{*} 10^{-4} \mathrm{~m}\right.$, $1.0 * 10^{-3} \mathrm{~m}$ und $1.5^{*} 10^{-3} \mathrm{~m}$ hinter der Kante) in der freien Scherschicht

Dieser Trend setzt sich nicht kontinuierlich in Strömungsrichtung fort. Da die Scherschicht konvektiv instabil ist, bildet sich im weiteren Verlauf eine Kelvin-HelmholtzInstabilität aus. Diese ist gekennzeichnet durch konvektives Wachstum, d.h. auch einer Zunahme der maximalen Scherschichtauslenkung. Charakteristisch ist die Bewegung in positive und negative y-Richtung um eine Ruhelage herum entgegen dem Relaxieren nahe der Kante in eine Ruhelage. Die Auftragung des 1ten Momentes in Abhängigkeit von der Zeit in Abbildung 5.4 zeigt dies exemplarisch für Schnitte $x=0.03 \mathrm{~m}$, $\mathrm{x}=0.04 \mathrm{~m}$ und $\mathrm{x}=0.05 \mathrm{~m}$ stromab der Hinterkante.

Das Auftreten der Instabilität als Folge einer Anregung ist in anderen Arbeiten (z.B. [Neuwald (1990)]) gezielt untersucht worden. In der vorliegenden Arbeit wird hauptsächlich der vom eigentlichen Ablöseprozess dominierte Orts- und Zeitbereich analysiert. 


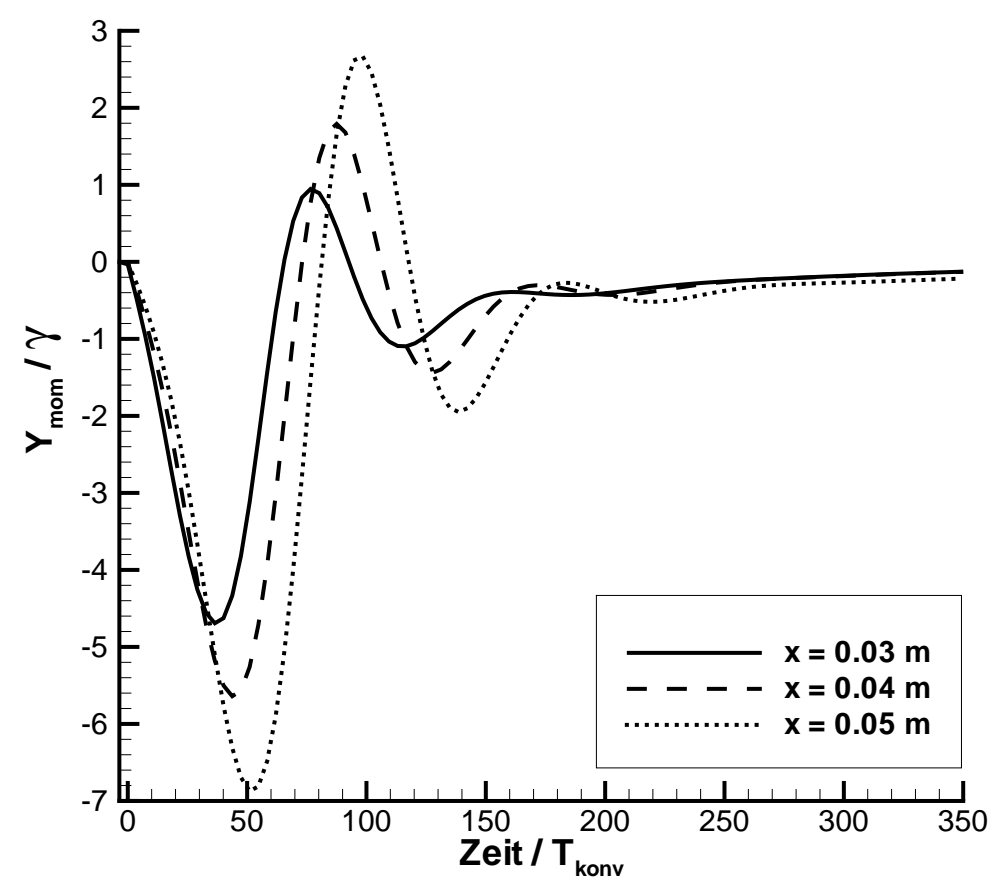

Abbildung 5.4: Das 1tes Moment der ausgelenkten Scherschicht (ohne Gleichanteil) zeigt das konvektive Anwachsen und zeitliche Abklingen einer Kelvin-Helmholtz-Instabilität.

\subsection{Auslenkungsprozess}

In diesem Abschnitt wird der Auslenkungsprozess, d.h. das Erreichen der maximalen Auslenkung der Wirbeligkeitsschicht quantitativ beschrieben. Das Maximum selbst weist eine Ortsabhängigkeit auf, wie in Abschnitt 5.3.1 dargestellt wird. Dabei bedarf die Kantenregion einer gesonderten Beschreibung, aufgrund des dominanten Einflusses von Reibungseffekten, wie in Abschnitt 5.3.2 erläutert wird. Eine Abschätzung des Wirkungsbereichs dieser Effekte ermöglicht ein Vergleich der Zeit des Eintretens der maximale Auslenkung mit der Zeitskala der Diffusion von Wirbeligkeit. Dies wird in Abschnitt 5.3.3 behandelt.

\subsubsection{Abhängigkeit der maximalen Auslenkung vom Messort}

Die Auslenkung der Scherschicht ist ein kontinuierlicher Prozess, der sowohl vom Ort x stromab der Hinterkante, als auch von der Zeit t nach der Anregung abhängt, wie bereits in Abschnitt 5.2 qualitativ beschrieben wurde. Um auch quantitative Aussagen hinsichtlich des Ortes, der Zeit und des Betrages machen zu können, muss ein ausgezeichnetes Stadium der Auslenkung identifiziert werden. Dazu eignet sich ihr Maxi- 
mum, welches für unterschiedliche Strömungen bestimmt wurde. In verschiedenen Schnitten bis $0.01 \mathrm{~m}$ hinter der Kante wurden aus dem zeitlichen Verlauf des 1ten Momentes, wie in Abbildung 5.3 dargestellt, jeweils das Minimum ermittelt, was der maximalen Auslenkung $\eta_{\max }$ entspricht. Abbildung 5.5 zeigt $\eta_{\max }$ in Abhängigkeit vom Ort x für verschiedene Strömungen.

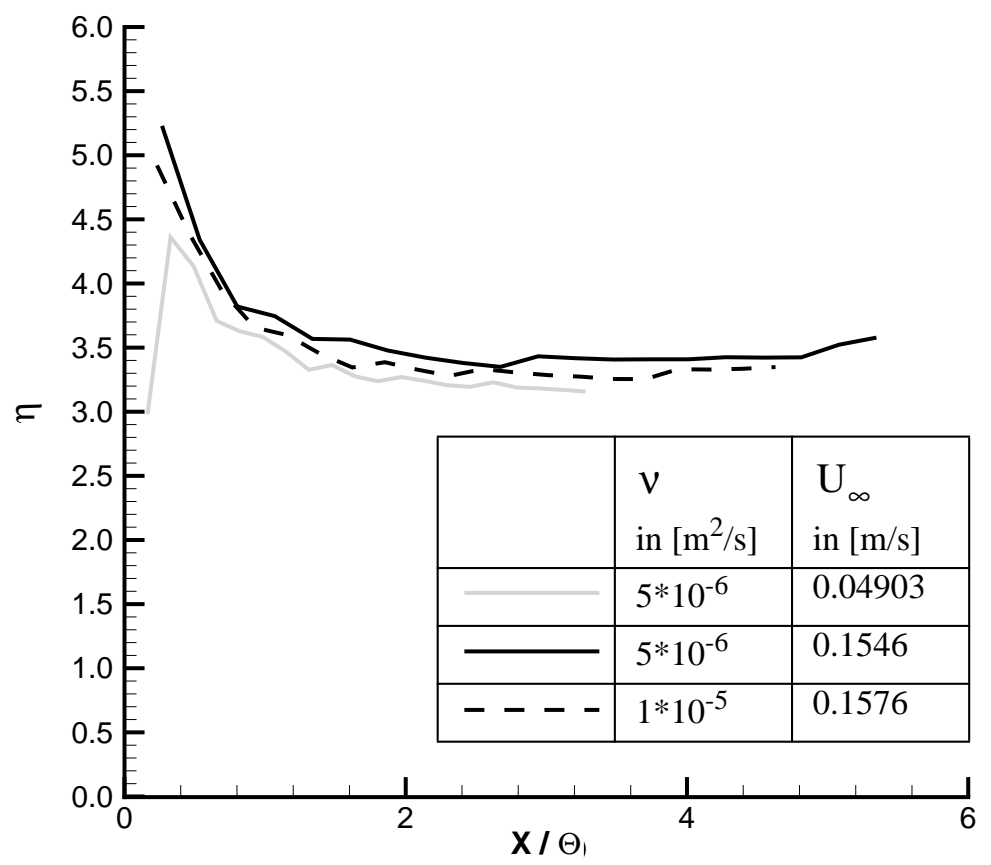

Abbildung 5.5: Scherschichtauslenkung in Abhängigkeit vom Ort x.

Obwohl sich die Hauptströmungsgeschwindigkeit der in Abbildung 5.5 verglichenen Simulationen um den Faktor drei und die Viskositäten um den Faktor fünf unterscheiden, ist die normierte Auslenkung am jeweiligen Ort nahezu identisch. Dies spricht dafür, dass die Normierung alle maßgeblichen Einflüsse berücksichtigt. Die ihr zugrunde liegenden Annahmen bezüglich des Zustandekommens der Auslenkung sind somit zutreffend.

Allgemein ist die Scherschicht nahe der Kante am stärksten ausgelenkt. Dabei ist zu berücksichtigen, dass für sehr geringe Abstände von der Spitze auch Reibungseffekte nicht zu vernachlässigenden Einfluss haben. Dies wird durch das Abweichen eines kantennahen Wertes in Abbildung 5.5 deutlich und ist Gegenstand des folgenden Abschnitts. 
Bis ca. $4 * 10^{-3} \mathrm{~m}$ geht die Maximalauslenkung mit dem Ort zurück. Ab ca. $1 * 10^{-2} \mathrm{~m}$ stromab der Hinterkante kann bereits der Einfluss der Kelvin-Helmholtz-Instabilität beobachtet werden, wie auch Abbildung 5.2 zeigt. Wäre dies nicht so, ist ein weiteres Abklingen der maximalen Scherschichtauslenkung in Strömungsrichtung zu erwarten. In dem in Abbildung 5.5 dargestellten Bereich jedoch kann an allen Orten ein signifikantes $\eta_{\text {max }}$ bestimmt werden.

\subsubsection{Effekte in der Kantenregion}

Die auslenkende Anregung entspricht einer Potenzialströmung. Für die Simulation einer reibungsbehafteten Strömung ist zusätzlich eine geeignete Wandanschlussbedinung implementiert worden (s. Abschnitt 4.2.1 auf Seite 46), die die Entwicklung einer Grenzschicht gestattet, wie sie sich an einer festen Oberfläche entwickelt. Diese wächst direkt an der Hinterkante ähnlich wie auf der Oberfläche der Platte mit der Zeit an. Die überlagerte Strömung wird hierdurch abgebremst. Das äußert sich in einer geringeren Scherschichtauslenkung an Orten $\mathrm{x}$ innerhalb der Grenzschicht gegenüber Positionen weiter stromab, wie z.B. Abbildung $5.3 \mathrm{zu}$ entnehmen ist.

Die in Abbildung 5.6 dargestellten $\mathrm{v}_{\Delta}$-Profile für $\mathrm{y}=0 \mathrm{zu}$ verschiedenen Zeiten stromab der Hinterkante zeigen deutlich den Einfluss der reibungsbehafteten Schicht. Nach der Theorie der Potenzialströmung sinkt der Betrag der $\mathrm{v}_{\Delta}$-Komponente stromab der Hinterkante bei $\mathrm{y}=0$ proportional $\mathrm{zu} \mathrm{x}^{-1 / 2}$. Diese Geschwindigkeitsverteilung hat ihr Maximum direkt an der Kante bei $\mathrm{x}=0$. Aufgrund der Haftbedingung ist dort die Geschwindigkeit jedoch null, wächst aber mit dem Abstand zur Wand an. Das resultierende Geschwindigkeitsprofil entlang einer Linie bei y $=0$ läßt sich somit durch den Einfluss dieser beiden Effekte beschreiben. Das eigentlich stationäre Feld der überlagerten Strömung ist im wandnahen Bereich zeitabhängig aufgrund der Zähigkeit und der damit verbundenen Diffusion von Wirbeligkeit.

Die Abweichung der simulierten Strömung von der reinen Potenziallösung verhält sich ähnlich wie die in Abschnitt 4.2.1 auf Seite 46 beschriebene Korrektur des Potenzialfeldes über einer festen Oberfläche. Die dort eingebrachten Voraussetzungen gelten an der Hinterkante nur bedingt. Zum einen ist Diffusion in mehr als eine Raumrichtung möglich und zum anderen ist die verwendete Gleichung (4.2) nur dann eine exakte Lösung der Navier-Stokes-Gleichung, wenn die Außenströmung von x unabhängig ist. 


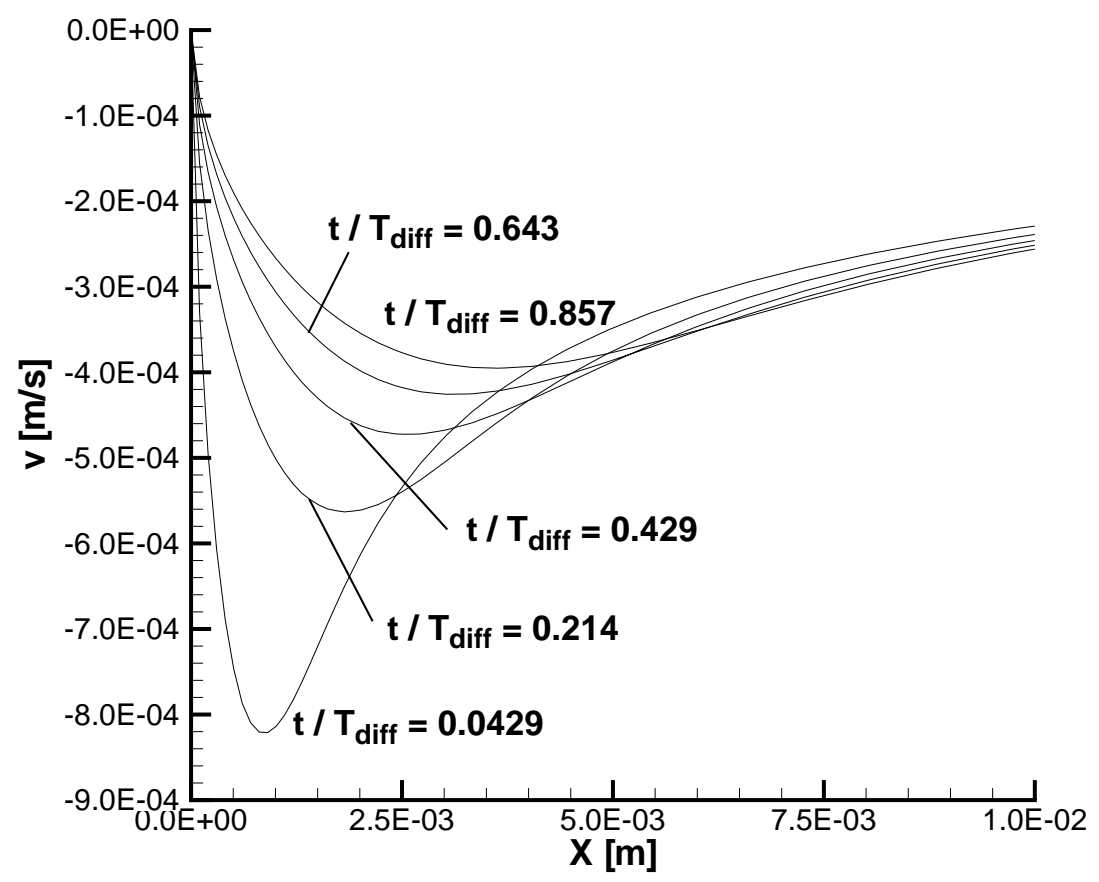

Abbildung 5.6: $\mathrm{v}_{\Delta}$-Profile für $\mathrm{y}=0$ und verschiedene Zeiten stromab der Hinterkante

Da hier lediglich eine Abschätzung des Effektes erfolgen soll, wird auf die in [Schlichting (1951)] beschriebenen weiteren Korrekturglieder verzichtet. Die Wirkung der Wandgrenzschicht skaliert somit nicht genau, aber in erster Näherung ebenfalls mit dem dimensionslosen Parameter $\xi$. In Abschnitt 5.3.3 wird auch auf die zeitliche Entwicklung dieses Effektes eingegangen.

\subsubsection{Zeitliche Entwicklung der maximalen Auslenkung}

Die Ausbreitung der maximalen Auslenkung, d.h der Zusammenhang zwischen dem Ort und der Zeit ihres Eintretens, ist in Abbildung 5.7 dargestellt. Aufgrund der normierung der Zeit mit der Konvektionszeit sind die verschiedene Strömungen gut vergleichbar. Die Ausbreitungsgeschwindigkeit der Auslenkung außerhalb der Kantenregion ist somit stark von der Geschwindigkeit der Hauptströmung abhängig.

Der sich ebenfalls zeitlich entwickelnde Einfluss der Kanten-Grenzschicht ist für einen Ort, an dem die Außenströmung aufgrund der Reibung um 1/e verringert wurde, in Abbildung 5.7 zusätzlich eingezeichnet. Nur für Orte unmittelbar an der Kante und entsprechend kleine Zeiten nach der Anregung ist die Wirkung der Grenzschicht dominant, wie bereits in vorhergehenden Abbildungen erkennbar war. 


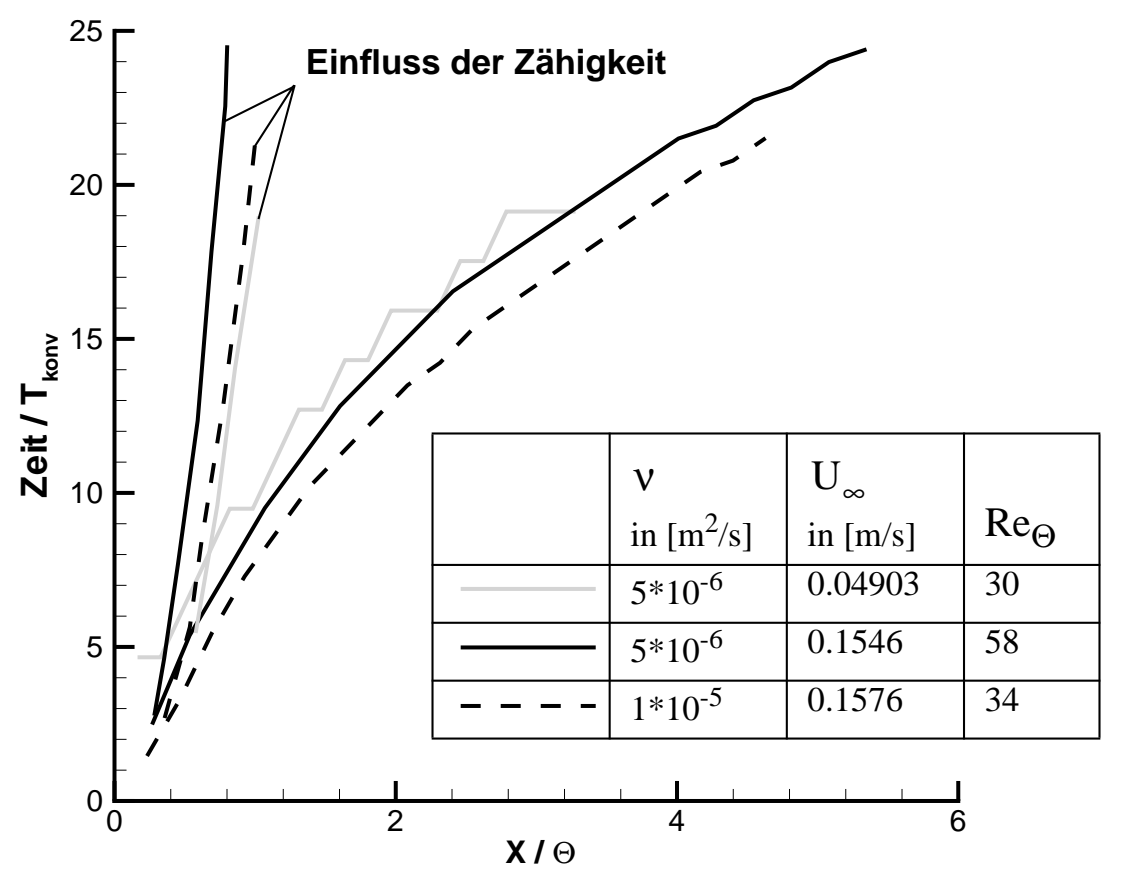

Abbildung 5.7: Dargestellt ist die Abhängigkeit der Zeit des Eintretens der max. Scherschichtauslenkung vom Ort. Der abgeschätzte Einfluss von Zähigkeitseffekten (s. Text) ist zum Vergleich eingezeichnet.

\subsection{Relaxationsprozess}

Die Rückkehr der Scherschicht in ihre Ruhelage ist der Prozess, welcher von besonderer praktischer Bedeutung ist. Die dafür benötigte Relaxationszeit bei gegebener Anregung läßt eine Abschätzung der Gültigkeit der quasistationären Kutta-Bedingung zu. Nach diese Zeit, ermittelt aus der experimentell bestimmten kritischen Strouhalzahl, richtet sich der zu simulierende Zeitraum und damit verbunden, gemäß den Stabilitätsüberlegungen aus Abschnitt 3.2.1 auf Seite 23 auch die Länge des Simulationsgebietes. In Abschnitt 5.4.1 ist dieser Sachverhalt ausgeführt.

Die einheitliche Formulierung einer Gesetzmäßigkeit, die das Abklingen der Scherschichtauslenkung in Abhängigkeit von der Relaxationszeit beschreibt, scheint nicht ohne weiteres möglich. Der Relaxationsprozess, als Antwort der Strömung auf die Störung, stellt sich als sehr komplex dar, wie seine Beschreibung anhand der Scherschichtauslenkung in Abhängigkeit von der Zeit in Abschnitt 5.4.2 zeigt. 


\subsubsection{Relaxationszeit}

Nach der Auslenkung der Strömung kehrt die Scherschicht innerhalb der Relaxationszeit in einen stationären Endzustand zurück. Ist die Periodendauer T einer (periodischen) Anregung größer als die Relaxationszeit $T_{\text {relax }}$, so löst die Wirbeligkeitsschicht in jedem Zeitpunkt nahezu tangential ab und es gilt die quasistationäre Kutta-Bedingung. In [Bechert (1988)], [Bechert \& Stahl (1988)] und [Graf (1998)] wurde eine kritische Strouhalzahl ermittelt:

$$
\mathrm{St}_{\Theta}=\frac{\mathrm{f} \Theta}{\mathrm{U}_{\infty}} \approx 0.005
$$

Für die Periodendauer T folgt daraus:

$$
\mathrm{T}=\frac{1}{\mathrm{f}} \approx \frac{\Theta}{0.005 \cdot \mathrm{U}_{\infty}} \geq \mathrm{T}_{\mathrm{kutta}} 2 \pi
$$

In Tabelle 5.1 ist diese obere Grenze von $\mathrm{T}_{\text {kutta }}$, die nicht zur Verletzung der quasistationären Kutta-Bedingung führt, für verschiedene Strömungen zusammengestellt. Ebenso wie die Zeit bis zum Erreichen der max. Auslenkung, als kürzeste Zeit, für die Wahl eines geeigneten Zeitschritts der instationären Simulation grundlegend ist, so ist die Relaxationszeit, als Gesamt-Simulationszeit, für die Anzahl der Zeitschritte maßgebend. Daraus folgend leitet sich aus ihr auch die Länge des Berechnungsgebietes ab. Dem liegt die Forderung zugrunde, dass eine Störung innerhalb der Simulationszeit nicht bis zum Ausströmrand konvektiert sein darf. Hierbei wird die Geschwindigkeit der Kernströmung als obere Grenze der Konvektionsgeschwindigkeit angenommen. Die somit aus dieser Geschwindigkeit und der Zeit $\mathrm{T}_{\text {kutta }}$ resultierende minimale Länge $1_{\text {min }}$ des Simulationsgebietes stromab der Hinterkante ist auch in Tabelle 5.1 aufgeführt. Für die Berechnung der Strömung mit ca. $15^{*} 10^{-2} \mathrm{~m} / \mathrm{s}$ Geschwindigkeit betrug die Länge des verwendeten Gitters $0.5 \mathrm{~m}$ und für Strömungen mit ca. $4.7 * 10^{-2} \mathrm{~m} / \mathrm{s}$ $0.9 \mathrm{~m}$ stromab der Hinterkante. Die Anforderungen an die Länge des Simulationsgebietes sind somit in jedem Fall erfüllt worden.

\subsubsection{Phasen der Relaxation}

Die Relaxation der Scherschicht, d.h. ihre Rückkehr in eine Ruhelage, ist in Abbildung 5.8 durch den zeitlichen Verlauf ihrer Auslenkung stromab der Hinterkante an einem Ort $x=5^{*} 10^{-3} \mathrm{~m}$ dargestellt. Es lassen sich zur Charakterisierung mehrere 
Tabelle 5.1: Relaxationszeit

\begin{tabular}{|l|l|l|l|l|}
\hline$v$ & $\begin{array}{l}\mathrm{U}_{\infty} \\
\text { in }\left[\mathrm{m}^{2} / \mathrm{s}\right]\end{array}$ & $\begin{array}{l}\Theta \\
\text { in }[\mathrm{m}]\end{array}$ & $\begin{array}{l}\mathrm{T}_{\text {kutta }} \\
\text { in }[\mathrm{s}]\end{array}$ & $\begin{array}{l}1_{\min } \\
\text { in }[\mathrm{m}]\end{array}$ \\
\hline $1 * 10^{-6}$ & $4.749 * 10^{-2}$ & $2.59 * 10^{-3}$ & 1.74 & 0.52 \\
\hline $5 * 10^{-6}$ & $4.903 * 10^{-2}$ & $3.05 * 10^{-3}$ & 1.97 & 0.61 \\
\hline $1 * 10^{-5}$ & $5.056^{*} 10^{-2}$ & $3.48 * 10^{-3}$ & 2.20 & 0.70 \\
\hline $5 * 10^{-6}$ & $15.46^{*} 10^{-2}$ & $1.87 * 10^{-3}$ & 0.385 & 0.37 \\
\hline $1 * 10^{-5}$ & $15.76^{*} 10^{-2}$ & $2.16^{*} 10^{-3}$ & 0.438 & 0.34 \\
\hline
\end{tabular}

Phasen abgrenzen, wie z.B. das Anwachsen der Auslenkung (A) bis zum Erreichen des Maximums. Dies war bereits Gegenstand vorheriger Abschnitte. Darauf folgt ein Rückgang (B). Dieser ist für verschiedene Strömungen auch an unterschiedlichen Messpositionen stets proportional der Konvektionszeit, wie die Ergebnisses mehrerer Simulationen gezeigt haben. Das erneute Anwachsen der Auslenkung stellt eine Art Störung (C) dar. Im weiteren Verlauf ist ein grundsätzlich andersartiges Abklingen (D) zu beobachten. Die resultierende „Diskontinuität“ der Relaxation verhindert die Formulierung einer einfachen mathematischen Beschreibung. Erste Ansätze dazu zeigten, dass sowohl verschiedene Zeitkonstanten, als auch unterschiedliche Abhängigkeiten der Auslenkung von diesen Konstanten berücksichtigt werden müssen. Das Ereignis, welches diese prinzipielle Änderung der Charakteristik der Relaxation nach sich zieht, wird im folgenden Kapitel anhand der Wechselwirbeligkeit genauer betrachtet. 


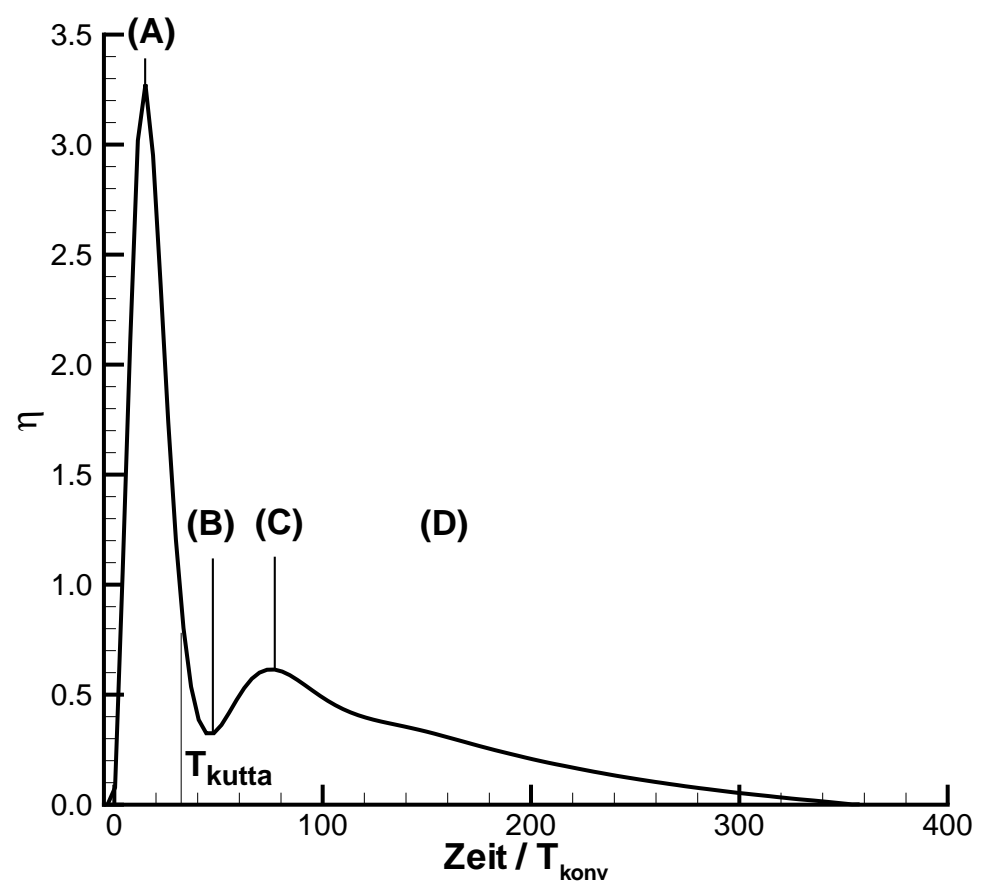

Abbildung 5.8: Zeitliche Entwicklung der Scherschichtauslenkung an einem Ort $\mathrm{x}=5^{*} 10^{-3} \mathrm{~m}$ stromab der Hinterkante während des gesamten Relaxationsprozesses. Die Buchstaben beziehen sich auf die im Text erläuterten Phasen der Relaxation. 


\section{Wechselwirbeligkeit}

Die Beschreibung des Relaxationsprozesses der Scherschicht anhand ihrer Auslenkung vermittelt ein erstes Bild über die Wirkungsweise der beteiligten Mechanismen. Diese ist jedoch so komplex, dass sie durch die Momente der Wirbeligkeitsverteilung nicht ausreichend beschrieben werden kann. Im Folgenden sind daher Zeitreihen abgebildet, die das gesamte Feld der Wechselwirbeligkeit in einem Bereich von $0.02 \mathrm{~m}$ x $0.02 \mathrm{~m}$ um die Hinterkante herum darstellen. Die zugrunde liegenden Daten entstammen der Simulation einer Strömung mit ca. $15^{*} 10^{-2} \mathrm{~m} / \mathrm{s}$ Hauptströmungsgeschwindigkeit eines Fluides mit $1 * 10^{-5} \mathrm{~m}^{2} / \mathrm{s}$ Viskosität, daraus ergibt sich eine Konvektionszeit von $\mathrm{T}_{\text {konv }}=0.01371 \mathrm{~s}$. Die Charakteristik des Relaxationsprozesses ist identisch der anderer Strömungen. Die phänomenologische Beschreibung dieser Sequenzen gibt weiteren Aufschluss der Reaktion der Scherschicht auf die eingebrachte Störung.

Die Wechselwirbeligkeit ist die gegenüber der stationären Grundströmung durch die Anregung veränderte Wirbeligkeit. Dies entspricht der Wirbeligkeitsverteilung des jeweiligen Zeitschritts nach Abzug der Verteilung vor der Anregung. Die Produktion der Wechselwirbeligkeit beruht auf zwei quasi unabhängige Mechanismen. Zum einen entsteht durch die Überlagerung der Potenzialströmung eine flächenhafte DeltaWirbeligkeitsverteilung an der Oberfläche der Splitterplatte. Diese Wirbeligkeit diffundiert und konvektiert mit der Zeit in die Strömung. Dies ist der durch das Modell (siehe „Modell der instationären Strömungsablösung“ auf Seite 11) beschriebene Anteil der Wechselwirbeligkeit. Zum anderen werden die Stromlinien der Gleichströmung durch die Anregung ausgelenkt. Wirbeligkeitsbehaftete Fluidelemente, die diesen folgen, bewirken einen Versatz der stationären Wirbeligkeitsverteilung der Gleichströmung. Auch dies liefert einen Beitrag zur Wechselwirbeligkeit.

Die Gruppierung der nachfolgend gezeigten Zeitschritte orientiert sich an den im Abschnitt 5.4.2 eingeteilten Phasen der Relaxation. Da die Wirkung der Anregung mit der Zeit stromab fortschreitet, bezieht sich diese Abgrenzung auf einen Ort x. Eine direkte Zuordnung der Wirbeligkeitsverteilung zu einem Punkt auf der Kurve des zeitlichen Verlaufs der Auslenkung in Abbildung 5.8 auf Seite 68 ist daher nur für die dort verwendete Position $5^{*} 10^{-3} \mathrm{~m}$ stromab der Kante möglich. Die Einteilung der Zeitschritte ist aus diesem Grund nur als Anhaltspunkt und Orientierung gedacht. 
Alle folgenden Darstellungen zeigen die Wechselwirbeligkeit in einem Bereich von $0.02 \mathrm{~m} \times 0.02 \mathrm{~m}$ um die Hinterkante unter Verwendung der Farbkodierung aus Abbildung 6.1.

$-0.02$

0.0

0.02

Abbildung 6.1: Farbkodierung der Felder der Wechselwirbeligkeit in $\left[\mathrm{m}^{2} / \mathrm{s}\right]$

\subsection{Auslenkung der Scherschicht}

Während der ersten Phase der instationären Scherschichtablösung erreicht die Wirbeligkeitsschicht ihre maximale Auslenkung. Das zugehörige Feld der Wechselwirbeligkeit ist für verschiedene Zeitpunkte in Abbildung 6.2 dargestellt.

$\mathrm{Zu}$ einem Zeitschritt $\mathrm{t}<0$ entspricht die Wirbeligkeitsverteilung der der stationären Gleichströmung. Da dieser Anteil abgezogen wird, ist die Wechselwirbeligkeit vor der Anregung im gesamter Bereich null.

In der Darstellung des ersten Zeitschritts nach der Anregung $\left(\mathrm{t} / \mathrm{T}_{\mathrm{konv}}=0.36\right)$, ist erkennbar, dass die neu entstandene negative Wirbeligkeit in einer dünnen Schicht an der Oberfläche der Platte konzentriert ist. Im weiteren Verlauf $\left(\mathrm{t} / \mathrm{T}_{\mathrm{konv}}=4.01\right)$ dehnt sich diese Schicht durch Diffusion und Konvektion aus. Zusätzlich ist auch positive Wirbeligkeit durch Verschiebung der stationären Verteilung entstanden. Da diese abgezogen wurde, zeigt der positive Anteil den Transport negativer Wirbeligkeit aus dieser Region heraus an. Die Wirbeligkeitsschicht ist somit nach unten versetzt worden, wie bereits im Anstieg der Auslenkung zu erkennen war.

Dieser Versatz nimmt im nächsten Zeitschritt $\left(\mathrm{t} / \mathrm{T}_{\mathrm{konv}}=7.66\right)$ weiter zu. Auffälliger jedoch ist die Entstehung eines scharf begrenzten Bereiches positiver Wechselwirbeligkeit an der Unterseite der Hinterkante. Seine Entwicklung ist in den Abbildungen der folgenden Phase dargestellt. 

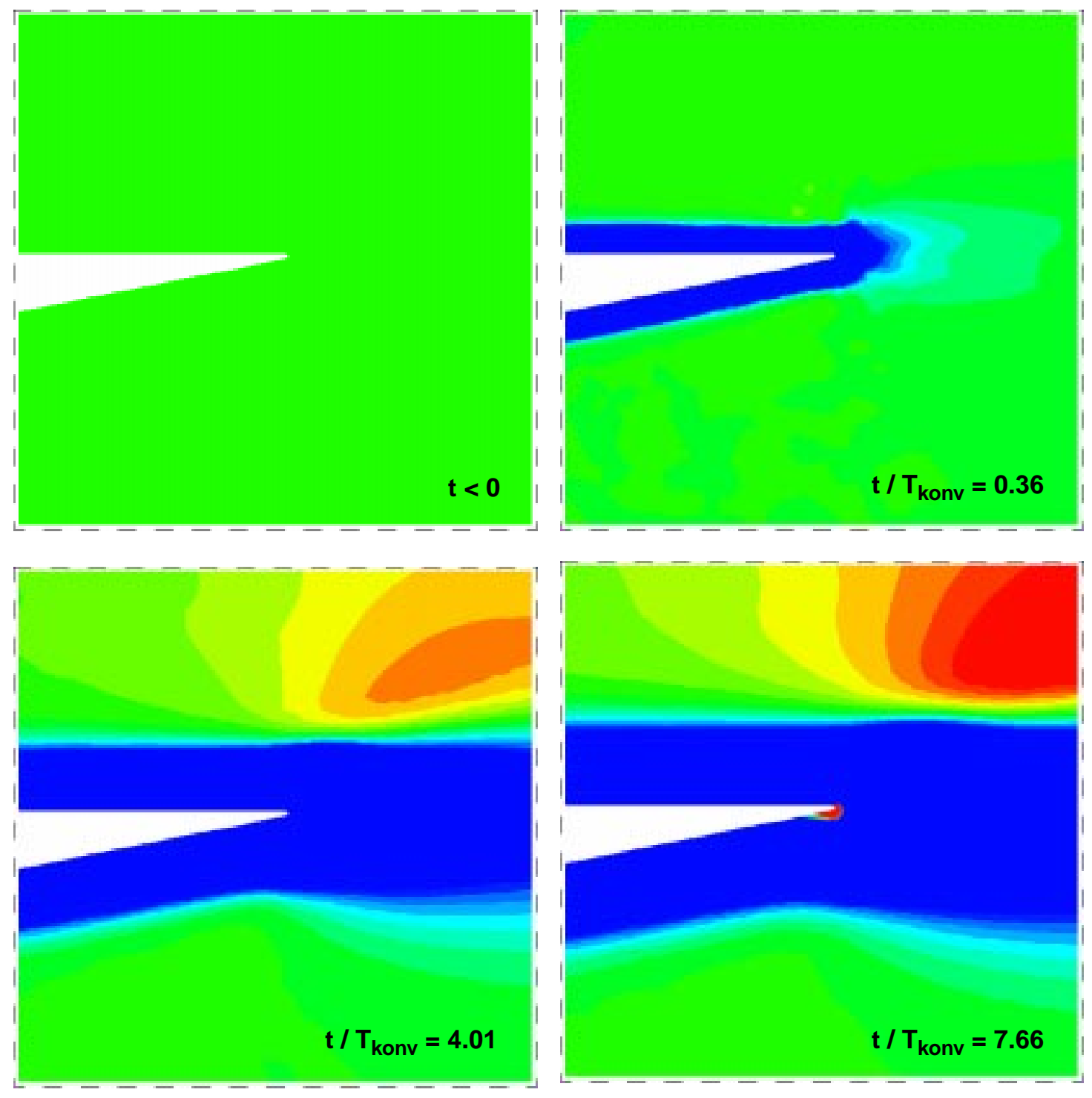

Abbildung 6.2: Wechselwirbeligkeit zu Zeitschritten während der Auslenkung der Scherschicht

\subsection{Aufreißen der Wirbeligkeitsschicht}

Der starke Rückgang der Scherschichtauslenkung in diesem Stadium (vgl. Abbildung 5.8 auf Seite 68) beruht auf dem Abschwimmen positiver Wirbeligkeit, wie in Abbildung 6.3 dargestellt. Dieser Bereich konvektiert stromab und dehnt sich durch Diffusion und Scherung weiter aus. Die Schicht negativer Wirbeligkeit wird dadurch aufgerissen. Die räumliche Verteilung der Wirbeligkeit ist nachhaltig verändert. Die Veränderung der Lage ihres Schwerpunktes ist somit nicht ohne weiteres mit den vorhergehenden, bzw. folgenden Stadien vergleichbar. Eine Charakterisierung des Relaxa- 

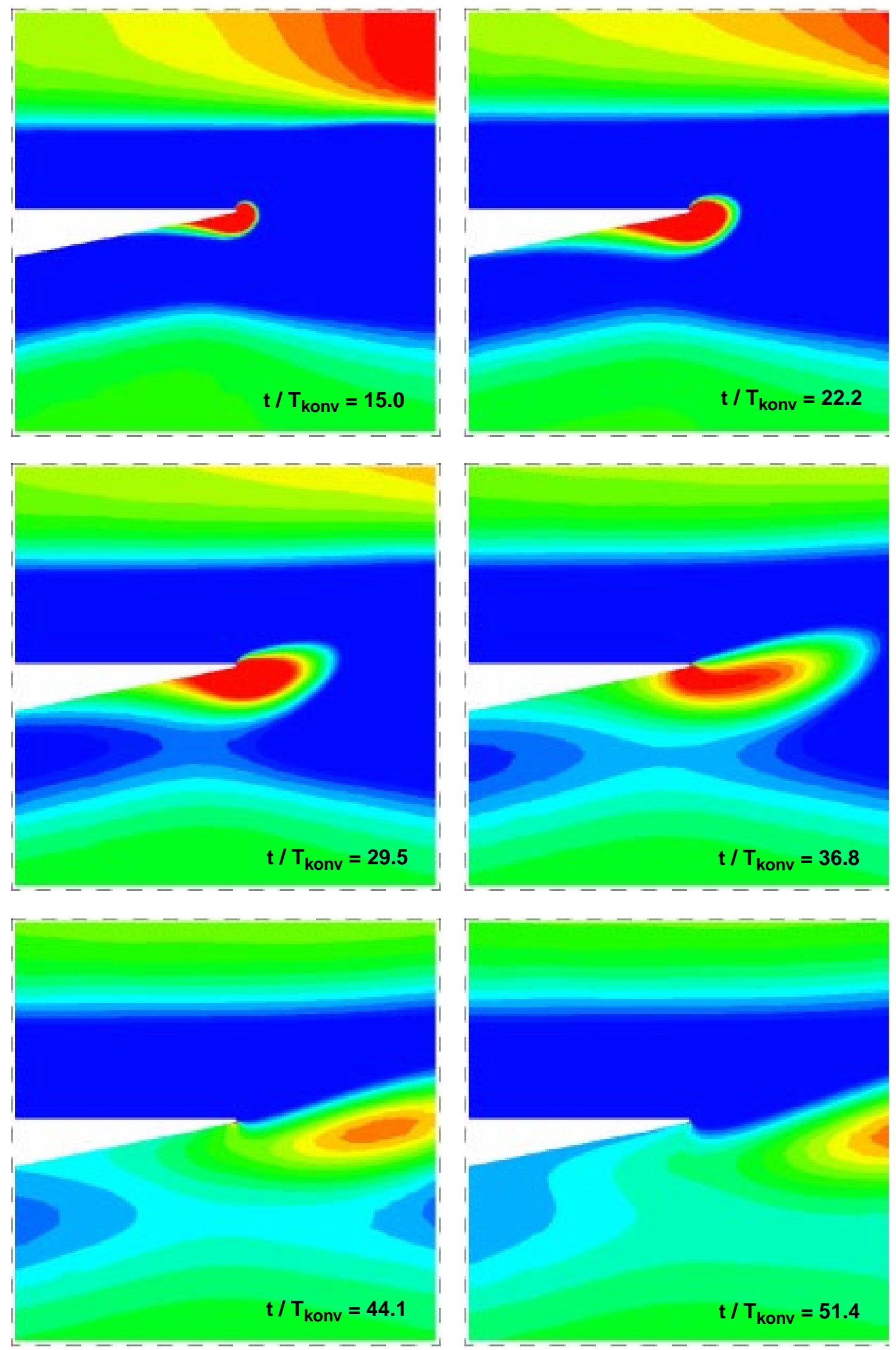

Abbildung 6.3: Felder der Wechselwirbeligkeit während des Aufreißens der Wirbeligkeitsschicht 
tionsprozesses anhand der Momente der Wirbeligkeitsverteilung ist durch das Auftreten dieses Ereignisses problematisch, wie bereits in Abschnitt 5 auf Seite 54 anklang.

Auch das Modell, welches in Abschnitt 2.3 auf Seite 11 vorgestellt wurde, stößt hier an Grenzen. Die Beschreibung der Relaxation der Scherschicht durch Umverteilung von Wirbeligkeit durch Konvektion und Diffusion trifft zwar auch für die in dieser Phase entstandene positive Wechselwirbeligkeit zu. Ihre Entstehung kann jedoch erst nach Erweiterung des Modells mit einbezogen werden. Ansatzpunkt hierzu ist die bereits in [Graf (1998)] aufgeworfene Frage, ob die Behandlung von Wirbeligkeit als passive Substanz allgemein zulässig ist. Wirbeligkeit ist eine lokale Eigenschaft der Strömung. Ihre Änderung beruht auf der lokalen Veränderung des Vektors der Strömungsgeschwindigkeit. Im Gegensatz dazu hat die Änderung der Konzentration einer passiven Substanz, wie z.B. Farbe, i.a. keine Rückwirkung auf die lokalen Strömungsverhältnisse. Es liegt die Vermutung nahe, dass der Staupunkt dadurch entsteht, dass die verschobene Gleichwirbeligkeit zu einer Strömung führt, die die Randbedingung an der Platte $(\overrightarrow{\mathrm{u}}=0)$ nicht mehr erfüllt.

Zur Entstehung der positiven Wechselwirbeligkeit geben die assoziierten Felder der gegenüber dem stationären Zustand veränderten Strömungsgeschwindigkeiten weiteren Aufschluss. Die Stromlinien des angeregten Feldes nach Abzug der Grundströmung sind in Abbildung 6.4 dargestellt. Der grundlegende Unterschied zum in Abschnitt 6.1 beschriebenen Stadium besteht in einer Richtungsänderung, also Rückströmung der Differenzgeschwindigkeit. Die nahezu potenzialtheoretische Umströmung der Kante unmittelbar nach der Anregung ist durch einen Wirbel aufgebrochen, der stromab konvektiert. 

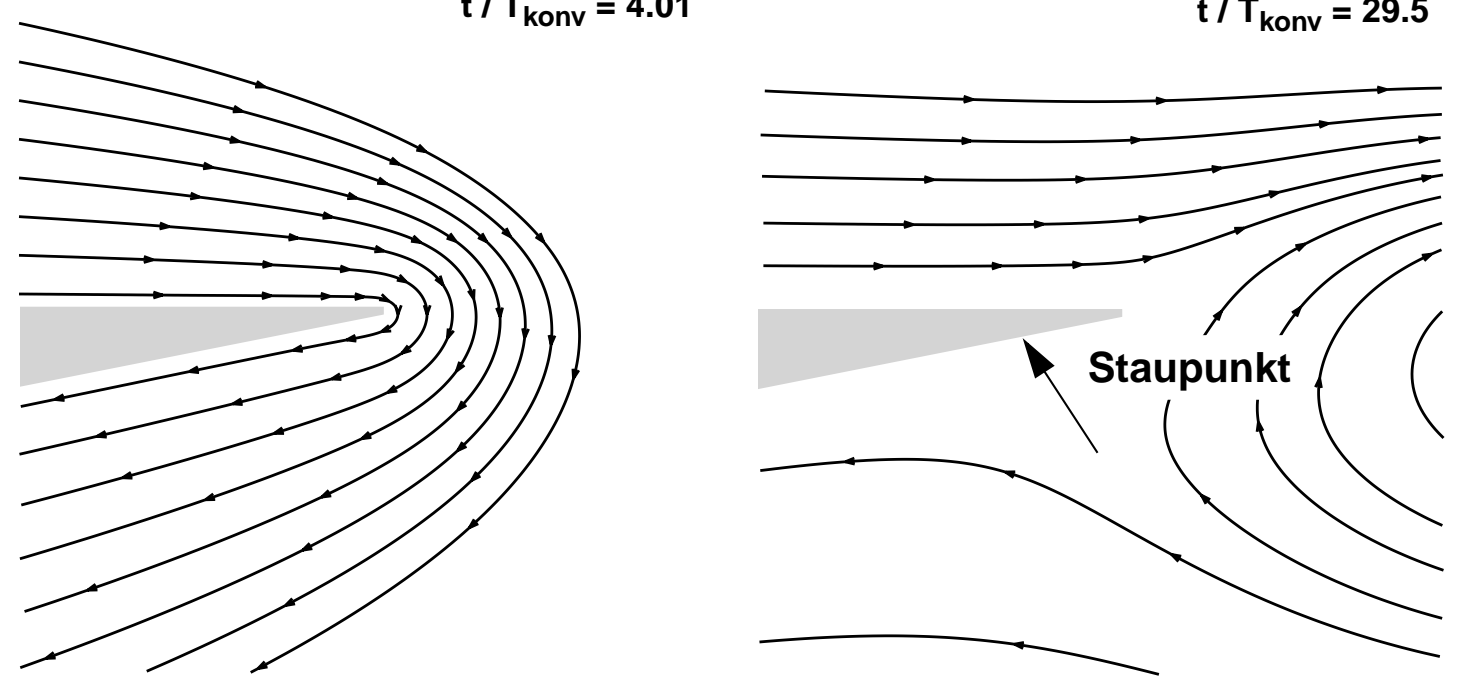

Abbildung 6.4: Stromlinien zu typischen Feldern der Wechselwirbeligkeit kurz nach der Anregung (links) und nach der Entstehung positiver Wechselwirbeligkeit an der Unterseite der Hinterkante (rechts)

\subsection{Rückschwingung der Scherschicht}

Die Schicht negativer Wirbeligkeit sinkt nach dem Durchzug des Gebietes der positiven Wirbeligkeit wieder ab, wie in Abbildung 6.5 dargestellt ist. Dieser Vorgang ist bewirkt ein erneutes Anwachsen der Auslenkung und entspricht daher einem Rückschwingen der Scherschicht, wie der zeitliche Verlauf ihrer Auslenkung in Abbildung 5.8 auf Seite 68 zeigt. Die nachfolgend einsetzende endgültige Relaxation der Scherschicht erfolgt wie vom Modell beschrieben durch Umverteilung von negativer Wirbeligkeit. Dieser Trend ist in Abbildung 6.5 aus dem Vergleich der Felder der Wechselwirbeligkeit der letzten beiden Zeitschritte $\left(\mathrm{t} / \mathrm{T}_{\text {konv }}=95.2\right.$ und $\left.\mathrm{t} / \mathrm{T}_{\text {konv }}=110\right)$ bereits erkennbar. 

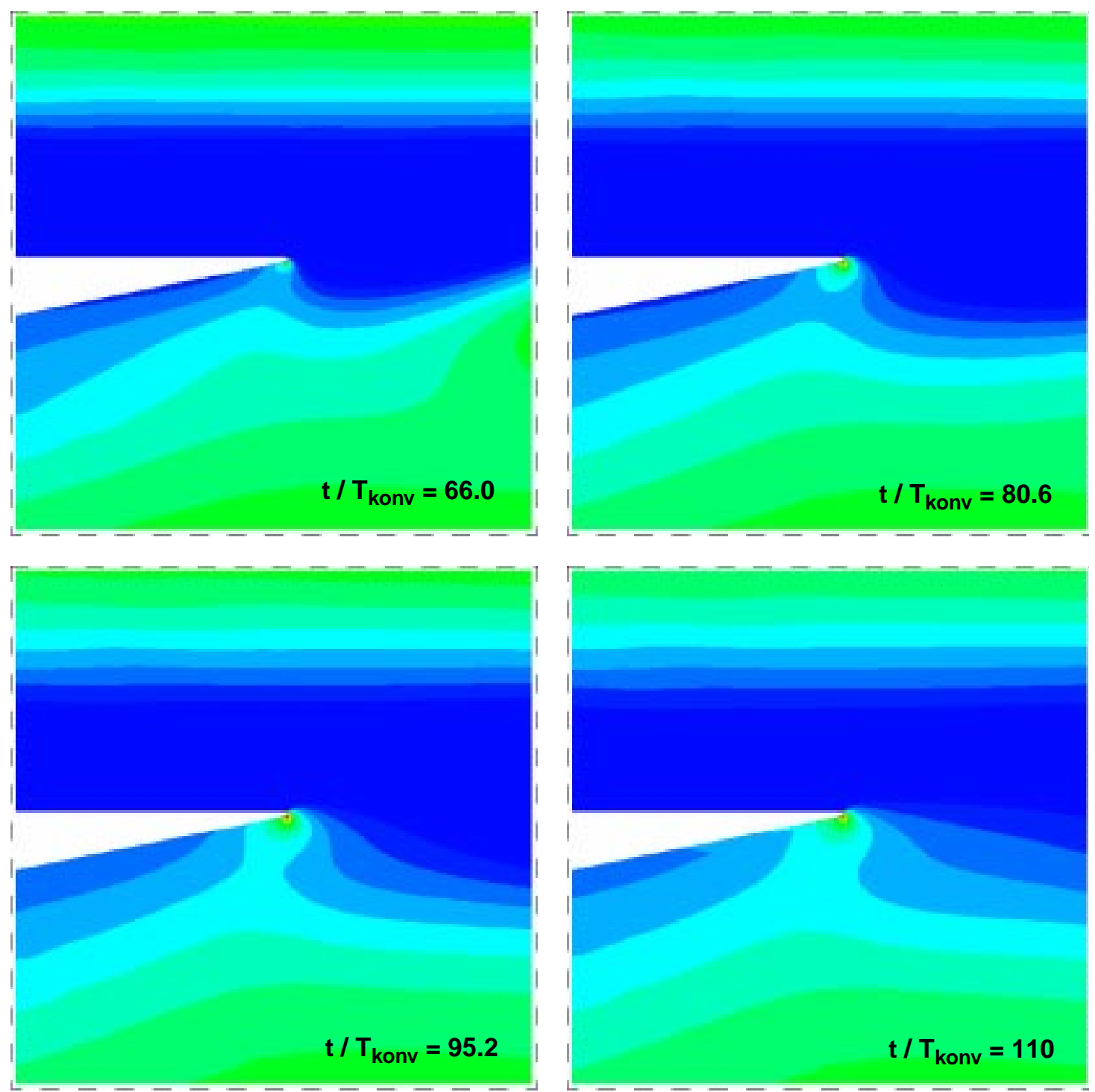

Abbildung 6.5: Die Folge der Felder der Wechselwirbeligkeit zeigt ein erneutes Absinken der Schicht negativer Wirbeligkeit nach dem Durchzug des Gebietes positiver Wirbeligkeit.

Im Vergleich zum vorhergehenden Stadium ist der Wirbel der Wechselgeschwindigkeiten weiter stromab konvektiert, wie die Stromlinien in Abbildung 6.6 (links) zeigen. Die Umströmung der Kante erfolgt weiträumiger. Sie ist erst im Zustand der nahezu vollständigen Relaxation Abbildung 6.6 (rechts) abgebaut. Die überlagerte Strömung fließt tangential ab, daher ist die Kutta-Bedingung wieder erfüllt. Die dazu notwendige Verteilung der Wechselwirbeligkeit ist im folgenden Abschnitt dargestellt. 


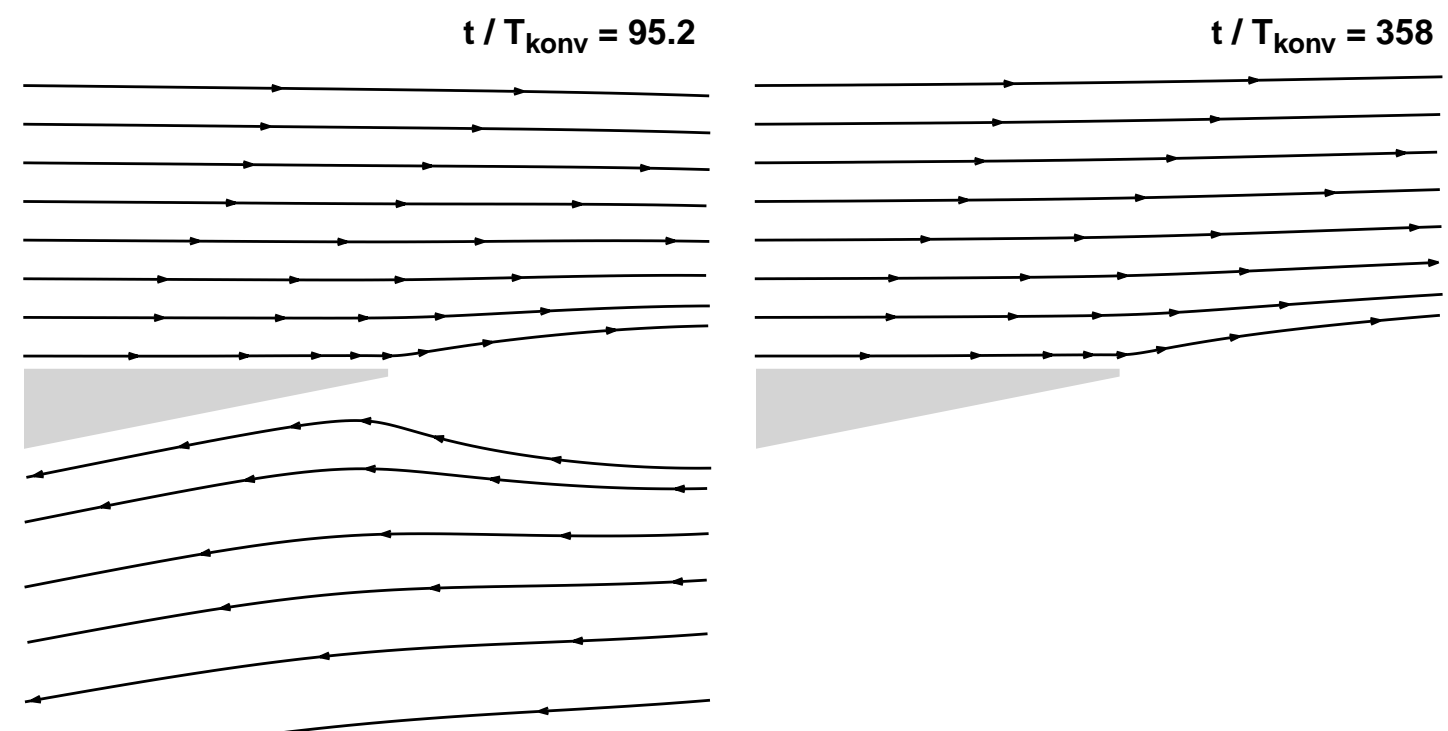

Abbildung 6.6: Stromlinien nach Konvektion des Wirbels der Wechselgeschwindigkeit (links) und nach fast vollständiger Relaxation der Scherschicht (rechts)

\subsection{Finale Relaxation}

Wie sich bereits in den letzten Zeitschritten der vorhergehend dargestellten Phase andeutete erfolgt die endgültige Relaxation der Scherschicht durch eine Umverteilung der Wechselwirbeligkeit von den Platte weg in die Strömung hinein. Abbildung 6.7 zeigt dies in einer Folge von Feldern der Wechselwirbeligkeit im letzten Stadium der Relaxation.

Die Verteilung der Wechselwirbeligkeit, bzw. deren Änderung, ist deutlich von der Wirkungsweise der elementaren Mechanismen der Konvektion und der Diffusion geprägt. Durch Konvektion wirbeligkeitsbehafteter Fluidelemente erfolgt ein Transport der Wechselwirbeligkeit in Hauptströmungsrichtung (vgl. z.B. Zeitschritt $\mathrm{t} / \mathrm{T}_{\mathrm{konv}}=285$ ). Durch Diffusion kommt es zu einer gleichmäßigen Abnahme der Wirbeligkeit an der Platte, wie der Vergleich aufeinander folgender Zeitschritte in Abbildung 6.7 deutlich zeigt. Die Schlussfolgerungen, die sich aus diesen und den Ergebnissen anderer Abschnitte ergeben, finden sich im folgenden Kapitel. 

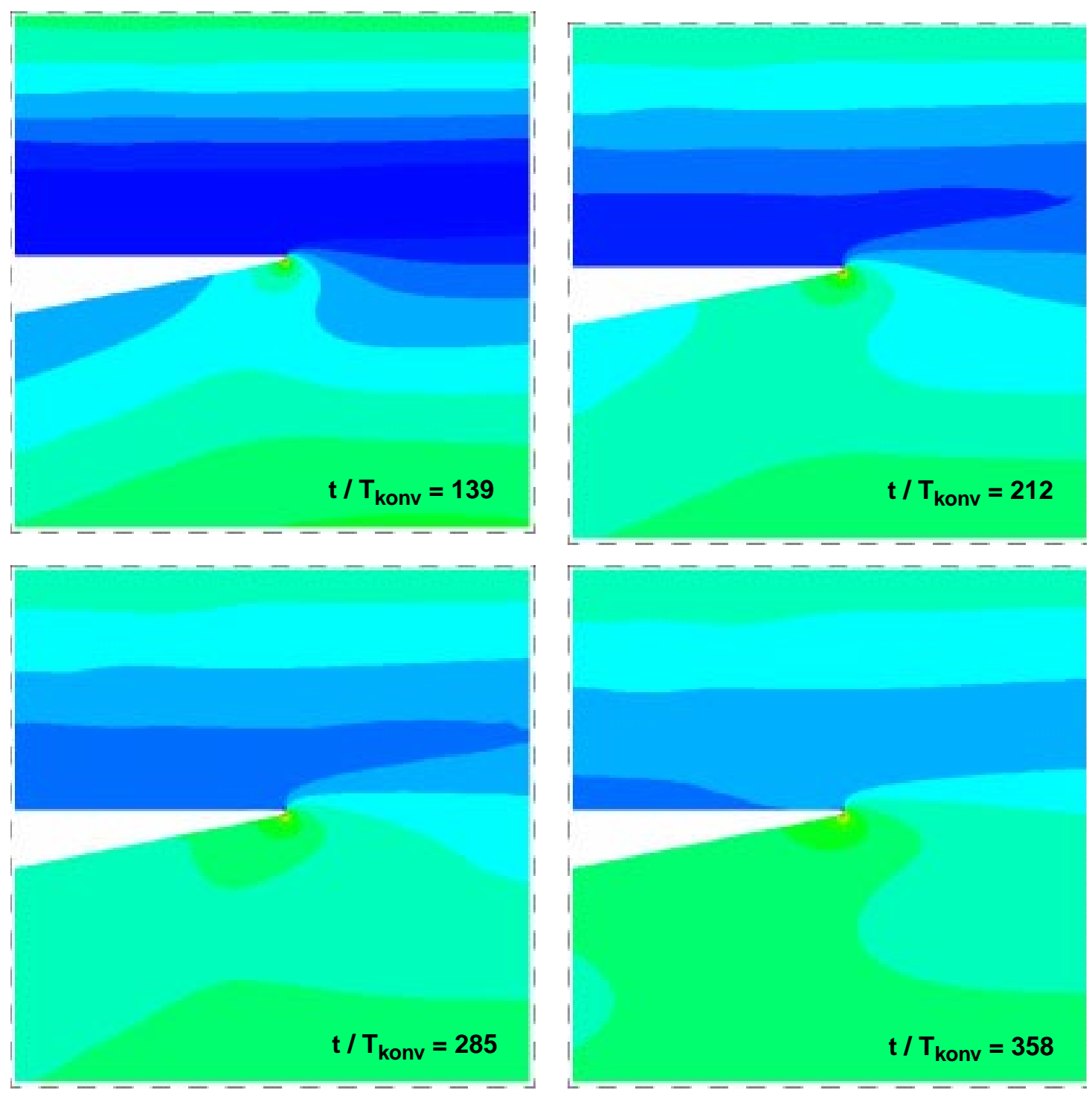

Abbildung 6.7: Endgültige Relaxation der Scherschicht durch Diffusion und Konvektion von Wechselwirbeligkeit von der Platte weg in die Strömung hinein 


\section{Zusammenfassung, Schlussfolgerungen und Ausblick}

Ziel dieser Arbeit war die Konzeption und Realisierung numerischer Simulationen zur Untersuchung der instationären Kutta-Bedingung für die Strömungsablösung einer Wirbeligkeitsschicht an einer Hinterkante. Diese Scherschicht sollte ausgelenkt und ihre Relaxation durch Umverteilung von Wirbeligkeit im Zeitbereich analysiert werden. Die dazu notwendige impulsartige Anregung führt zu großen Gradienten, besonders im zu untersuchenden Bereich der Hinterkante. Daraus resultieren hohe Anforderungen an die räumliche Auflösung der numerischen Strömungsberechnung, die im Experiment i.a. nicht erreicht werden kann. Um diese zu gewährleisten, wurden mehrere problemspezifische Studien durchgeführt, in denen die Eigenschaften des Zusammenwirkens von Rechengitter und Strömungslöser bestimmt wurden. Auf diese Weise konnte sichergestellt werden, dass das Simulationsergebnis nicht von Programmparametern oder numerischen Artefakten abhängig ist. Da ein im DLR neuentwickelter Strömungslöser verwendet wurde und die Simulationen dieser Arbeit mit zu seinen ersten Anwendungen gehörten, erfolgte auch eine Validierung nach der DFGBenchmark-Studie. Es konnte gezeigt werden, dass die erreichbare Genauigkeit ausreichend ist, um das Problem zu lösen.

Nach diesen Vorarbeiten erfolgte die zunächst getrennte Simulation der stationären Gleichströmung und des später zu überlagernden anregenden Feldes. Die Grundströmung wurde zur besseren Vergleichbarkeit der Ergebnisse aus Experimenten abgeleitet. Dies konnte durch Einströmrandbedingungen erreicht werden, die aus der gesonderten Berechnung einer Kanalgeometrie stammen, welche die Strömungsverhältnisse des für die Experimente verwendeten Wasserkanals nachbildet. Damit die Anregung der durch einen Druckgradienten erfolgten Anregung entspricht, soll das überlagerte Feld die Eigenschaften einer Potenzialströmung haben, d.h. im Innern wirbelfrei sein. Am Rand soll durch eine Anschlussbedingung die Diffusion von Wirbeligkeit, entstanden in einer infinitesimal dünnen Schicht an festen Oberflächen, in die Strömung hinein nachgebildet werden. Dies konnte durch die Vorgabe zeitabhängiger Einströmrandbedingungen realisiert werden, wie die Eigenschaften der berechneten Strömung zeigen. 
Die Ergebnisse der Simulationen der Anregung der Scherschicht wurden zunächst anhand des 1ten Momentes der Wirbeligkeitsverteilung nach Abzug der Verteilung der Grundströmung analysiert. Das Moment dient zur Quantifizierung der Auslenkung der Wirbeligkeitsschicht. Zur besseren Vergleichbarkeit der Resultate aus den Berechnungen unterschiedlicher Strömungen wurde eine Normierung eingeführt. Diese erwies sich als sehr gut anwendbar, was zeigt, dass die dort eingebrachten Annahmen über die Abhängigkeit der Auslenkungsamplitude von den Parametern der Gleichströmung und der Anregung zutreffend sind.

Die anschließende Untersuchung der zeitlichen Ausbreitung der Anregung stromab der Hinterkante zeigte, dass sich mehrere Bereiche unterscheiden lassen. In einer Region unmittelbar an der Kante sind Reibungseffekte aufgrund der Grenzschicht dominant. Diese Region wurde gesondert von dem angrenzenden Bereich der ungestörten Scherschichtauslenkung untersucht. Die zeitliche Entwicklung der Einflüsse der jeweils maßgeblichen Effekte wurde in einem Vergleich der Ausbreitungsgeschwindigkeiten von Grenzschicht und maximaler Scherschichtauslenkung gegeneinander abgeschätzt. Weiter stromab kommt es zu Kelvin-Helmholtz-Instabilitäten, die bereits Gegenstand anderer Arbeiten sind.

Aus dem zeitlichen Verlauf des 1ten Momentes nach impulsartiger Anregung der Strömung konnte die aus Experimenten mit periodischer Anregung bekannte Relaxationszeit der Strömung nicht ermittelt werden. Die Rückkehr der Wirbeligkeitsschicht in einen stationären Endzustand erfolgte nicht monoton. Daher ließ sich die Dynamik der Scherschicht nicht durch eine einfache mathematische Formulierung mit der Relaxationszeit als Parameter beschreiben. Wie die Analyse des Feldes der Wechselwirbeligkeit für eine Folge von Zeitschritten zeigt, ist dieser Sachverhalt in der Entstehung positiver Wirbeligkeit begründet. Diese führt zu einer Störung der Umverteilung der negativen Wirbeligkeit der Scherschicht. Der Relaxationsprozess nach einer impulsartigen Anregung läßt sich somit nicht ausschließlich durch Konvektion und Diffusion von Wirbeligkeit beschreiben, die zum Zeitpunkt $\mathrm{t}=0$ an der Plattenoberfläche erzeugt wird. 


\section{Schlussfolgerung}

Die Produktion von positiver Wirbeligkeit innerhalb einer Schicht negativer Wirbeligkeit als Reaktion der Strömung auf die Anregung kann nicht mit dem vorgestellten Modell erklärt werden. Die vereinfachte Betrachtung der Wirbeligkeit als Transportgröße ist in diesem Fall nicht zulässig. Durch Umverteilung einer passiven Substanz wird die Strömung nicht beeinflusst. Wirbeligkeit hingegen leitet sich aus den lokalen Strömungsgeschwindigkeiten ab, die Veränderung ihrer Verteilung wirkt daher auch auf die Strömung. Dieser Sachverhalt wird in dem Modell bisher nicht nachgebildet, ist jedoch zur allgemeinen Beschreibung der Relaxation einer Wirbeligkeitsschicht zu berücksichtigen, wie die Simulationen zeigen.

\section{Ausblick}

Nachdem u.a. die notwendigen Voraussetzungen für die zuverlässige Berechnung der instationären Scherschichtablösung im Rahmen dieser Arbeit geschaffen wurden, bietet gerade die numerische Simulation gute Möglichkeiten weiteren Aufschluss über die Art der notwendigen Modellerweiterung zu erhalten. Es erscheint sinnvoll, zunächst in einer Studie die Produktion von positiver Wirbeligkeit in Abhängigkeit der charakteristischen Zeiten des Modells genauer zu untersuchen. Da die Störung der Relaxation an der Hinterkante der Platte entsteht, verspricht die Analyse z.B. der Wandschubspannung einen Ansatzpunkt zum Vergleich mit der quasistationären Kutta-Bedingung. Die Verfügbarkeit des Drucks an jedem Gitterpunkt bietet eine weitere Möglichkeit die Bedingungen für die Gültigkeit der verschiedenen Ablösebedingungen zu untersuchen.

Um eine bessere Vergleichbarkeit der vom Modell beschriebenen Umverteilung der Wirbeligkeit mit der Wechselwirbeligkeit der Simulation zu erzielen, sollten deren Anteile je nach Entstehungsursache separiert werden. Es sollte eine Trennung der Wechselwirbeligkeit entstanden an der Platte von der durch die Verschiebung der stationären Strömung entstandenen Wechselwirbeligkeit erfolgen. Dazu muss letztere während des gesamten Relaxationsprozesses separat als passive Transportgröße behandelt werden. Die nach der Anregung an der Oberfläche der Platte entstandene Wirbeligkeit kann dann aus der Wechselwirbeligkeit nach Abzug der umverteilten Gleichwirbeligkeit zum jeweiligen Zeitpunkt erhalten werden. Da die betrachteten 
Effekte - bezogen auf die Grundströmung - im Promillebereich liegen, ist zuvor durch sorgfältige Validierung die Genauigkeit der Lösung der Transportgleichungen zu bestimmen.

Die numerische Simulation ermöglicht die vergleichsweise einfache Bestimmung des Einflusses unterschiedlicher Geometrieparameter (vgl. [Chen \& Ho (1987)]), wie z.B. der Plattendicke oder des Öffnungswinkel der Hinterkante. Die Berechnung der Strömung über eine ebene Platte gestattet z.B. einen vereinfachten Vergleich der Simulationsergebnisse mit theoretischen Vorhersagen. 


\section{Literaturverzeichnis}

B. C. Basu, G. J. Hancock

The unsteady motion of a two-dimensional aerofoil in incompressible inviscid flow

J. Fluid Mech., vol 87, pp. 159-178, 1978

D. W. Bechert

Sound absorption caused by vorticity shedding, demonstrated with a jet flow

J. of Sound and Vib. (1980), 70(3), pp. 389-405

D. W. Bechert

Excitation of instability waves in free shear layers

Part 1. Theory

J. Fluid Mech., vol 186, pp. 47-62, 1988

D. Bechert, U. Michel

The Control of a Thin free Shear Layer with and without a Semi-Infinite Plate by a Pulsating Flow Field

Acoustica, 1975, Vol. 33, No. 5, pp. 287-307

D. Bechert, E. Pfitzenmaier

Optical compensation measurement on the unsteady exit condition at a nozzle discharge edge

J. Fluid Mech., 1975, vol. 71, pp. 123-144

D. W. Bechert, B. Stahl

Excitation of instability waves in free shear layers.

Part 2. Experiments

J. Fluid Mech., 1988, Vol. 186, pp. 63-84

S.-H. Chen, C.-M. Ho

Near Wake of an Unsteady Symmetric Airfoil

J. Fluid and Structures (1987) 1, pp. 151-164

T. Colonius

Numerically Nonreflecting Boundary Conditions

AIAA Paper 96-1661, 1996 
D. G. Crighton

The Kutta Condition in Unsteady Flow

Ann. Rev. Fluid Mech. 1985, 17, pp. 411-445

P. G. Daniels

On the Unsteady Kutta Condition

Quaterly Journal of Mechanics and Applied Mathematics, 1978, Vol. 31, pp. 49-75

C. Dierken

Rezeptivität einer ablösenden Strömungsscherschicht bei hohen Frequenzen

Diplomarbeit, III. Physikalisches Institut, Univ. Göttingen, 1995

L. Enghardt

Adaptive Unterdrückung der Kelvin-Helmholtz-Instabilität einer zweidimensionalen laminaren Scherschicht

Diss. Math.-Nat- Fak. Univ. Göttingen, 1996

J. H. Ferziger, M. Peric

Computational Methods for Fluid Dynamics

2. Auflage, Springer Verlag, 1997

R. Graf

Instationäre Strömungsablösung und Wirbeltransport an der Hinterkante einer Splitterplatte

Diplomarbeit, III. Physikalisches Institut, Univ. Göttingen, 1998

M. Griebel, T. Dornseifer, T. Neunhoeffer

Numerische Simulation in der Strömungsmechanik

Vieweg, 1995

V. Grützmacher

Unterdrückung von Instabilitätswellen in einer dreidimensionalen abgelösten Scherschicht

Diss. Math.-Nat- Fak. Univ. Göttingen, 1993 
K. Hannemann

Numerische Simulation und stabilitätstheoretische Untersuchung des absolut und konvektiv instabilen Nachlaufs

DFVLR-Forschungsbericht 88-09, 1988

M. S. Howe

A Review of the Theory of Trailing Edge Noise

J. of Sound and Vib. (1978), 61(3), pp. 437-465

von Karman Institute (VKI)

Introduction to Computational Fluid Dynamics

Lecture Series, Jan. 1999

R. Kessler

Numerische Gitter und deren Eigenschaften

Kursunterlagen zu NUMET '91, Numerische Methoden zur Berechnung von Strömungs- und Wärmeübergangsproblemen, 21.-24.10.91 in Erlangen

U. B. Mehta

Credible Computational Fluid Dynamics Simulations

AIAA Journal, Vol. 36, No. 5, May 1998, pp. 665-667

U. B. Mehta

Guide to Credible Computer Simulations of Fluid Flows

Journal of Propulsion and Power, Vol. 12, no. 5, 1996, pp. $940-948$

P. Neuwald

Anregung der Kelvin-Helmholtz-Instabilität durch eine einlaufende Tollmien-Schlichting-Welle

Diss. Math.-Nat.-Fak. Univ. Göttingen, 1990

S. A. Orszag, S. C. Crow

Instability of a Vortex Sheet Leaving a Semi-Infinite Plate

Stud. in Appl. Math., 1970, Vol. XLIX, No. 2, pp. 167-181

D. R. Poling, D. P. Telionis

The Response of Airfoils to Periodic Disturbances -

The Unsteady Kutta Condition

AIAA-Journal, 24(2), pp. 193-199, 1986 
L. Prandtl, K. Oswatitsch, K. Wieghardt

Führer durch die Strömungslehre

9. Auflage, Vieweg, 1990

J. Rebel

Aktive Beeinflussung einer ablösenden dreidimensionalen Plattengrenzschicht mittels Tollmien-Schlichting-Wellen

Diss. Math.-Nat- Fak. Univ. Göttingen, 1992

J. Rebel, D. Ronneberger

The Effect of Shear Stress on the Propagation and Scattering of Sound in Flow Ducts

J. of Sound and Vib.; 1992; 158(3); p. 469-496

S. W. Rienstra

Sound diffraction at a trailing edge

J. Fluid Mech. (1981), vol. 108, pp. 443-460

A. Rizzi, J. Vos

Towards Establishing Credibility in Computational Fluid Dynamics Simulations

AIAA Journal, Vol. 36, No. 5, May 1998, pp. 668-675

D. Ronneberger

Theoretische und experimentelle Untersuchung der Schallausbreitung durch Querschnittssprünge und Lochplatten in Strömungskanälen

Abschlußbericht zum DFG-Forschungsvorhaben „Akustische Reflexion und Transmission von Einbauten in durchströmten Kanälen“, Göttingen, 1987

D. Ronneberger

Experimentelle Untersuchungen zum akustischen Reflexionsfaktor von unstetigen Querschnittsänderungen in einem luftdurchströmten Rohr

Acustica, Vol. 19, 1967/68, pp. 222-235

B. Satyanarayana, S. Davis

Experimental Studies of Unsteady

Trailing-Edge Conditions

AIAA Journal, Vol. 16, No. 2, February 1978, pp. 125-129 
M. Schäfer, S. Turek

Benchmark Computations of Laminar Flow

Around a Cylinder

in E.H. Hirschel (editor), Flow Simulation with High-

Performance Computers II, Volume 52 of Notes on Numerical

Fluid Mechanics, pp. 547-566, Vieweg, 1996

H. Schlichting

Grenzschicht-Theorie

Verlag G. Braun, Karlsruhe, 3. Auflage, 1951

D. Schwamborn, T. Gerhold, R. Kessler

DLR-TAU Code - an Overview

1st ONERA/DLR Aerospace Symposium, ONERA, pp. S4-2 - S4-10, 1999

J. H. Spurk

Strömungslehre

Springer-Verlag, 4. Auflage, 1996

M. Türkyilmazoglu, J. S. B. Gajjar, A. I. Ruban

The Absolute Instability of Thin Wakes in an Incompressible/Compressible Fluid

Theoret. Comput. Fluid Dynamics 13 (1999), pp. 91-114

H. Wedekind, G. Görz, R. Kötter, R. Inhetveen

Modellierung, Simulation, Visualisierung:

$\mathrm{Zu}$ aktuellen Aufgaben der Informatik

Informatik-Spektrum 21 (1998), pp. 265-272, Springer-Verlag 


\section{A. Anhang}

\section{A.1 Charakterisierende strömungsphysikalische Größen}

In den folgenden Abschnitten werden die in dieser Arbeit mehrfach verwendeten strömungsphysikalischen Größen und ihre Definition kurz erläutert.

\section{Wirbeligkeit}

Bei der Interpretation der gewonnen Ergebnisse ist die Wirbeligkeit eine wichtige charakterisierende Größe. Sie ist zu verstehen als eine auf einen Punkt und nicht auf ein Gebiet bezogene, lokale Wirbelstärke und ist im Zweidimensionalen definiert gemäß:

$$
\omega=\frac{\partial v}{\partial x}-\frac{\partial u}{\partial y}
$$

\section{Impulsverlustdicke}

Die Impulsverlustdicke quantifiziert die Verminderung des Impulses durch Abbremsen der Strömung in der Grenzschicht. Anschaulich ist dies die Breite einer an der Wand gelegenen Schicht, durch die eine ideale, reibungsfreie Flüssigkeit strömt, deren Impulsfluss pro Zeiteinheit mit dem Impulsverlust übereinstimmen, den eine realen Strömung infolge der Reibungswirkung innerhalb der Grenzschicht erfährt.

Die Impulsverlustdicke $\Theta$ ist definiert als:

$$
\Theta=\int_{0}^{\infty} \frac{\mathrm{u}}{\mathrm{U}}\left(1-\frac{\mathrm{u}}{\mathrm{U}}\right) \mathrm{dy}
$$

\section{Momente der Wirbeligkeitsverteilung}

Einige Eigenschaften der Scherschicht können gut anhand der Momente ihrer Wirbeligkeitsverteilung beschrieben werden. Das nullte Moment $\Omega_{\text {mom }}(\mathrm{x})$ gibt die Gesamtwirbeligkeit in Abhängigkeit der x-Koordinate an. Es ist definiert als:

$$
\Omega_{\text {mom }}(\mathrm{x})=\int_{-\infty}^{\infty} \omega(\mathrm{x}, \mathrm{y}) \mathrm{dy}
$$


Die Lage des Schwerpunkts der Verteilung wird durch das erste Moment angegeben:

$$
\mathrm{Y}_{\mathrm{mom}}(\mathrm{x})=\frac{1}{\Omega_{\mathrm{mom}}(\mathrm{x})} \int_{-\infty}^{\infty} \omega(\mathrm{x}, \mathrm{y}) \mathrm{ydy}
$$

Das zweite Moment ist ein Maß für die Breite der Verteilung:

$$
\left(\delta_{\text {mom }}(\mathrm{x})\right)^{2}=\frac{1}{\Omega_{\mathrm{mom}}(\mathrm{x})} \int_{-\infty}^{\infty} \omega(\mathrm{x}, \mathrm{y})\left(\mathrm{y}-\mathrm{Y}_{\mathrm{mom}}(\mathrm{x})\right)^{2} \mathrm{dy}
$$

Da die Momente der Wirbeligkeit nicht im gesamten Simulationsgebiet erfasst werden sollen, sondern nur der Bereich der zu charakterisierenden Scherschicht maßgeblich ist, sind geeignete, d.h. physikalisch sinnvolle Integrationsgrenzen zu verwenden. Wie bereits in [Neuwald (1990)], so wurde auch hier vom Minimum der Geschwindigkeit im Nachlauf der Platte bis über die Plattengrenzschicht in die Hauptströmung hinein integriert. Das Minimum des Geschwindigkeitsprofils entspricht einem Vorzeichenwechsel des Wirbeligkeitsprofils. In Abbildung A.1 ist der Zusammenhang zwischen der u-Komponente und der Wirbeligkeit exemplarisch in einem Schnitt stromab der Hinterkante dargestellt.

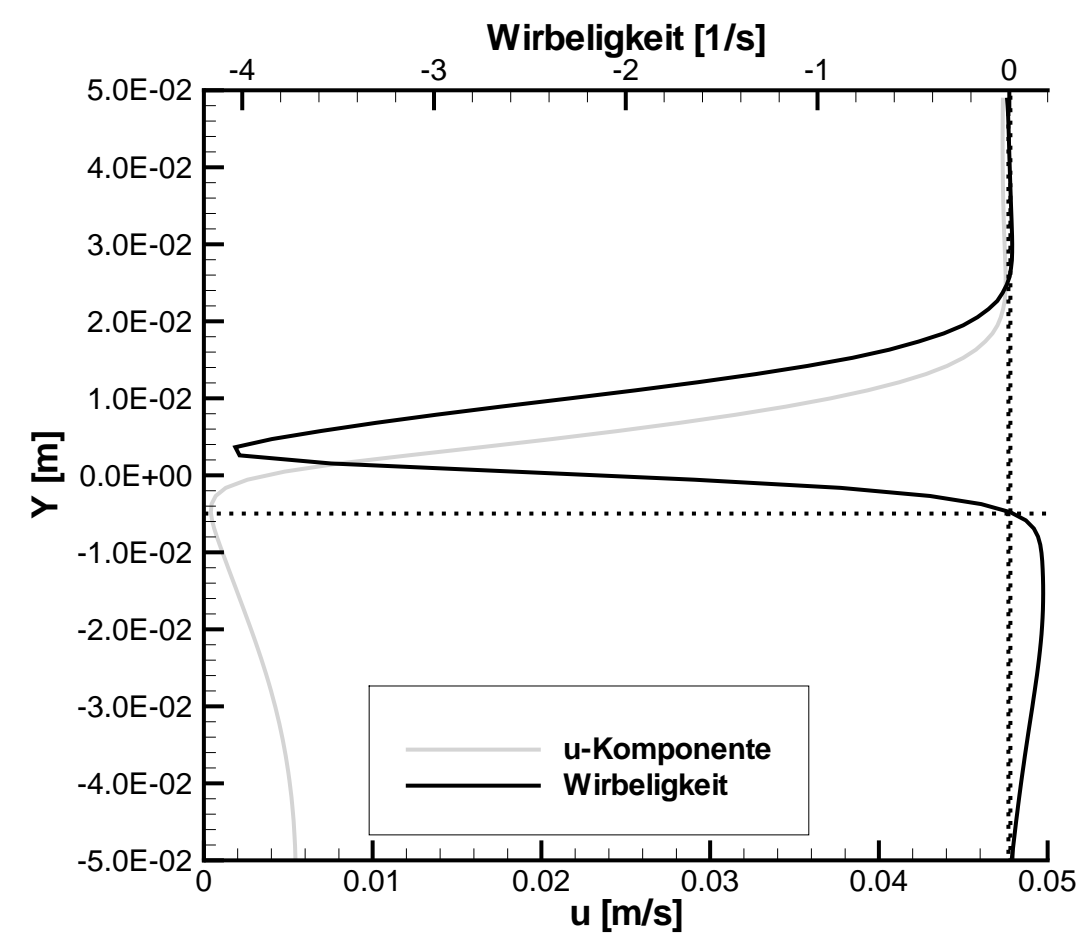

Abbildung A.1: u-Komponente und Wirbeligkeit im Nachlauf der Platte $\left(10^{-2} \mathrm{~m}\right.$ stromab der Kante) zur Veranschaulichung der Wahl der unteren Integrationsgrenze bei der Bestimmung der Momente der Wirbeligkeitsverteilung 


\section{A.2 Validierung nach der DFG-Benchmarkstudie}

Der $\tau^{\mathrm{i}}$-Code lag in einer ersten für Anwender freigegebenen Version vor. Da aus diesem Grund noch keine ausreichenden Erfahrungen und Vergleichsstudien zur Qualität der Lösung verfügbar waren, wurde eine Validierung nach der DFG-Benchmarkstudie [Schäfer \& Turek (1996)] durchgeführt. Eine Abschätzung der Genauigkeit der Simulation ist besonders vor dem Hintergrund der Bearbeitung einer Problematik aus der Grundlagenforschung notwendig, da nicht auf vielfältige Validierungsexperimente oder aber Simulationsergebnisse anderer Gruppen zurückgegriffen werden kann.

Es ist somit nachzuweisen, dass die erreichte Genauigkeit, bzw. Zuverlässigkeit der Simulation für ihre spezielle Anwendung zur Untersuchung der vorliegenden Fragestellung ausreicht. Dies läßt sich unter zwei Aspekten betrachten, zum einen der numerischen Genauigkeit und zum anderen der strömungsmechanischen Genauigkeit. $\mathrm{Zu}$ deren Quantifizierung sind unterschiedliche Methoden anzuwenden, wie in Abbildung A.2 dargestellt [Mehta (1996)].

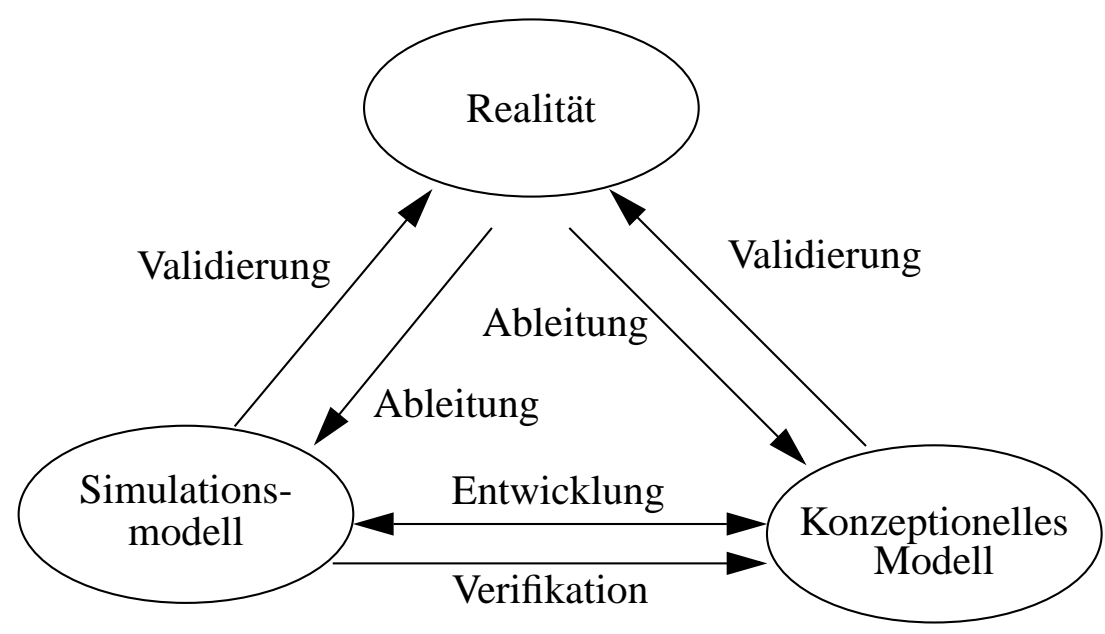

Abbildung A.2: Zusammenhang zwischen Realität, konzeptionellem Modell und Simulationsmodell und deren Verknüpfungen untereinander

Die Verläßlichkeit einer Simulation wird in Verifikations- und Validierungsprozessen gemessen. Verifikation bestimmt die Zuverlässigkeit der Simulation, unter dem Aspekt ihrer konkreten Anwendung. Hierzu wird der Grad abgeschätzt, in dem das Simulationsmodell eine genaue Repräsentation des konzeptionellen Modells, d.h. der Beschreibung der Strömung durch die Navier-Stokes-Gleichungen, darstellt [Mehta (1998)]. 
Die Verifikation muß zeigen, dass das Simulationswerkzeug unter Verwendung gegebener numerischer Parameter, wie Randbedingungen und Gitterpunkten, die zugrunde liegenden, die Physik beschreibenden Gleichungen, korrekt löst [Rizzi \& Vos (1998)]. Validierung hingegen führt zu einer Abschätzung des Grades, in dem die Realität korrekt wiedergegeben wird. Validierung stellt sicher, dass ein richtiges Ergebnis erreicht wird und Verifikation, dass das Ergebnis richtig erreicht wird [Mehta (1998)].

Es ist darüberhinaus notwendig, die Unsicherheit, mit der die Simulation behaftet ist, zu bestimmen, d.h Fehler zu quantifizieren. Im mathematischen Sinne ist ein Fehler definiert als die Differenz einer beobachteten, bzw. berechneten Größe zu ihrem wahren Wert. Ist nun aber dieser wahre Wert unbekannt oder unsicher bestimmt, kann der Fehler nur abgeschätzt werden. Das entspricht der Bestimmung der Unsicherheit der Simulation.

Zur Beurteilung der Qualität der Simulation und damit auch deren Zuverlässigkeit, ist eine Validierung unumgänglich.

\section{A.2.1 Allgemeine Spezifikationen}

Von der Deutschen Forschungsgemeinschaft (DFG) wurde eine Studie gefördert, die einen Überblick über die Ergebnisse verschiedener Strömungslöser von Universitäten und Industrieunternehmen ermöglicht [Schäfer \& Turek (1996)]. Die verwendeten Programme nutzen z.T. neuerer Verfahren und Algorithmen, wie unstrukturierte Gitter, den Mehrgitteralgorithmus, Gebietszerlegung, Gitteranpassung (Adaption), deren Vorteile und deren Anforderungen hinsichtlich Genauigkeit und Effizienz untersucht werden sollten. Voraussetzung für die Vergleichbarkeit der Ergebnisse ist eine genaue Definition der Rand- und Startbedingungen. Daher wurde als Testfall zur Lösung der inkompressiblen Navier-Stokes-Gleichung die zwei- und dreidimensionale Simulation der laminaren Zylinderumströmung gewählt. Die in dieser Arbeit durchgeführten Simulationen sind ausschließlich zweidimensional. Im Folgenden werden daher die Ergebnisse des $\tau^{\mathrm{i}}$-Codes für die zweidimensionalen Testfälle vorgestellt. 
Der Zylinder befindet sich nicht ganz in der Mitte eines Kanals (s. auch Abbildungen des Rechengitters weiter unten) und wird von einer parallelen Kanalströmung angeströmt. Das verwendete Medium ist ein inkompressibles Newtonsches Fluid, für welches die Massen- und Impulserhaltung gilt. Die kinematische Viskosität ist definiert als $v=10^{-3} \mathrm{~m}^{2} / \mathrm{s}$ und die Dichte ist $\rho=1.0 \mathrm{~kg} / \mathrm{m}^{3}$. Die Abmessungen und die Randbedingungen des Berechnungsgebietes sowie weitere Definitionen sind in [Schäfer \& Turek (1996)] dokumentiert.

Um die Richtigkeit der Simulation verifizieren zu können, bzw. die Fehler quantifizieren zu können, sind geeignete, die Strömung beschreibende Größen aus den Simulationsdaten zu extrahieren. Dabei muss sicher gestellt werden, dass sich eventuelle Fehler beim Lösen der verschiedenen Gleichungen tatsächlich in einem Abweichen der Vergleichsgröße von einem als richtig erachteten Wert niederschlagen. Innerhalb welcher Grenzen ein Wert als korrekt angenommen wird, kann je nach Validierungsfall aus dem Vergleich mit experimentell gewonnenen Messwerten, für einige ausgewählte Strömungen aus theoretischen Berechnungen oder aber durch den Vergleich der Ergebnisse mehrerer numerischer Simulationen unterschiedlicher (validierter) Programme erfolgen.

Da verschiedene Teilprogramme, z.B. zur Lösung der Impulsgleichung oder zur Lösung der Druckgleichung oder zur Interpolation der Daten zwischen den Mehrgitterebenen, unterschiedliche Fehler in der Lösung verursachen können, sind i.a. mehrere Größen abzuleiten. In dieser Studie wird die Charakteristik der simulierten Strömung u.a. durch folgende Größen (jeweils in SI-Einheiten) beschrieben:

Tabelle A.1: Vergleichsgrößen

\begin{tabular}{|l|l|}
\hline Symbol & Beschreibung \\
\hline $\mathrm{c}_{\mathrm{D}}$ & Widerstandskoeffizient des Zylinders \\
\hline $\mathrm{c}_{\mathrm{L}}$ & Auftriebskoeffizient des Zylinders \\
\hline $\mathrm{L}_{\mathrm{a}}$ & Länge des Ablösegebietes hinter dem Zylinder \\
\hline$\Delta \mathrm{P}$ & Druckdifferenz zwischen Vorder- und Rückseite des Zylinders \\
\hline $\mathrm{St}$ & Strouhalzahl des instationären Zylindernachlaufs \\
\hline
\end{tabular}


Um den Einfluss des Gitters auf das Ergebnis abschätzen zu können, wurden die Simulationen für drei verschieden feine räumliche Diskretisierungen durchgeführt. Abbildung A.3 zeigt das gröbste, Abbildung A.4 das mittelfeine und Abbildung A.5 das feinste Rechengitter.

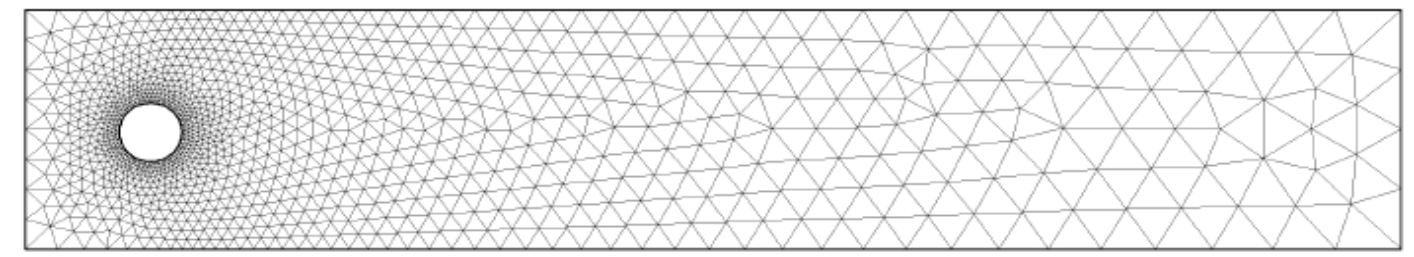

Abbildung A.3: Grobes Gitter für die DFG-Benchmark-Studie

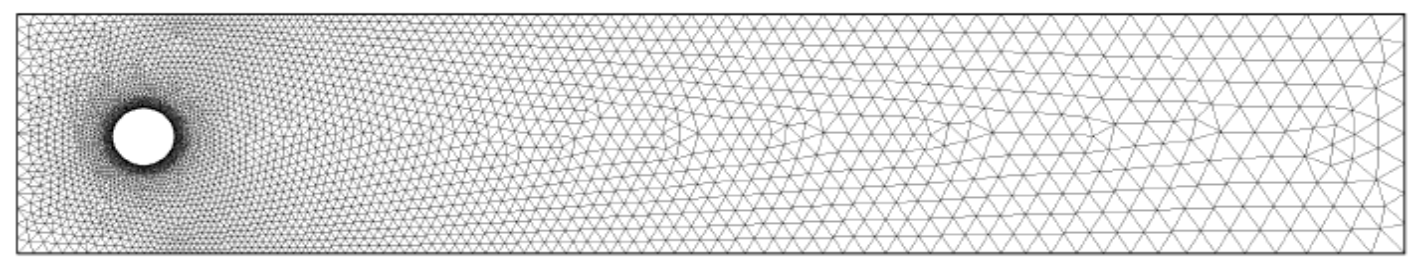

Abbildung A.4: Mittelfeines Gitter für die DFG-Benchmark-Studie

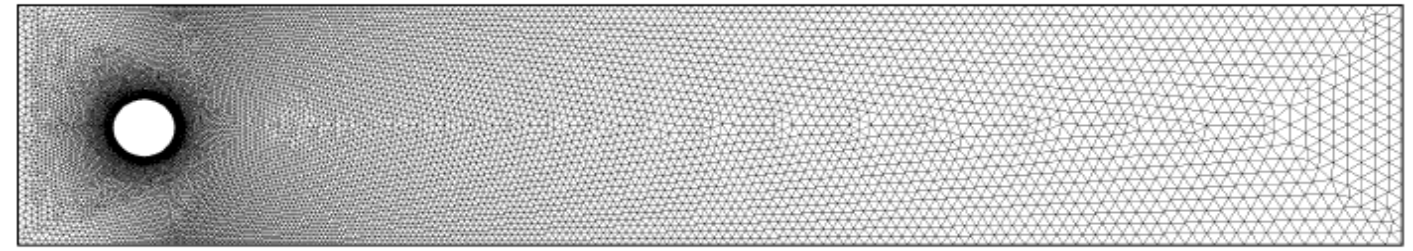

Abbildung A.5: Feinstes Gitter für die DFG-Benchmark-Studie

\section{A.2.2 Stationäre Strömung}

Eine mittleren Einströmgeschwindigkeit von $0.3 \mathrm{~m} / \mathrm{s}$ führt zu einer stationären Zylinderumströmung, wie in Abbildung A.6 dargestellt. Die Reynoldszahl, gebildet mit dem Durchmesser des Zylinders ist in diesem Fall 20.

In Tabelle A.2 finden sich die strömungsphysikalischen Vergleichsgrößen und die empfohlenen Grenzen, innerhalb derer diese Werte liegen sollten. Das >- und das <Zeichen geben ggf. an, ob der aus der Simulation berechnete Wert oberhalb bzw. unterhalb des Intervalls der richtigen Größen liegt. Mit dem Übergang vom groben über das 


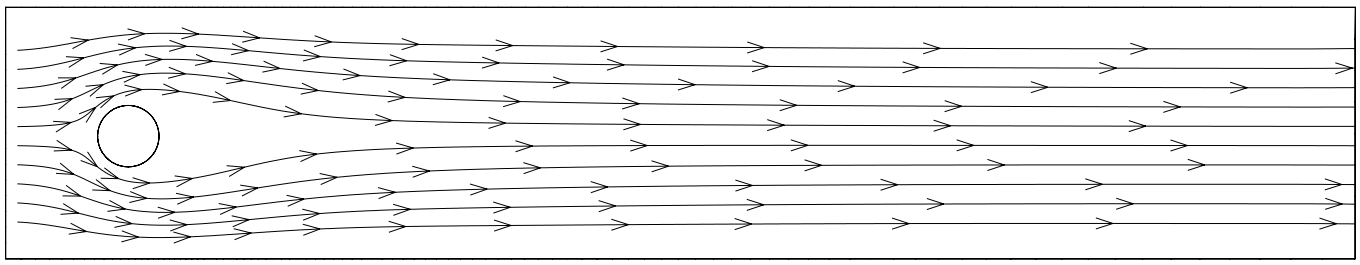

Abbildung A.6: Stromlinien der stationären Zylinderumströmung

mittelfeine zum feinen Gitter verbessern sich alle Vergleichsgrößen. Für das feine Gitter liegen die Werte ausnahmslos innerhalb der Grenzen, d.h. die Validierung ist für den stationären zweidimensionalen Fall in allen Aspekten erfolgreich.

Tabelle A.2:Stationärer Fall 2D-1

\begin{tabular}{|l|l|l|l|l|}
\hline Gitterpunkte & $\mathrm{c}_{\mathrm{D}}$ & $\mathrm{c}_{\mathrm{L}}$ & $\mathrm{L}_{\mathrm{a}}$ & $\Delta \mathrm{P}$ \\
\hline \hline 27162 & 5.5755 & 0.0106 & 0.0849 & 0.1172 \\
\hline 7502 & $5.5666<$ & 0.0109 & 0.0849 & $0.1179>$ \\
\hline 2390 & $5.5252<$ & $0.01261>$ & $0.0822<$ & $0.1187>$ \\
\hline \hline Untere Grenze & 5.5700 & 0.0104 & 0.0842 & 0.1172 \\
\hline Obere Grenze & 5.5900 & 0.0110 & 0.0852 & 0.1176 \\
\hline
\end{tabular}

\section{A.2.3 Instationäre Strömung}

Bei einer Reynoldszahl von 100 entwickelt sich eine instationäre Zylinderumströmung. Dies wird bei einer mittleren Einströmgeschwindigkeit von $1.5 \mathrm{~m} / \mathrm{s}$ erreicht und ist (für einen Zeitpunkt) in Abbildung A.7 dargestellt.

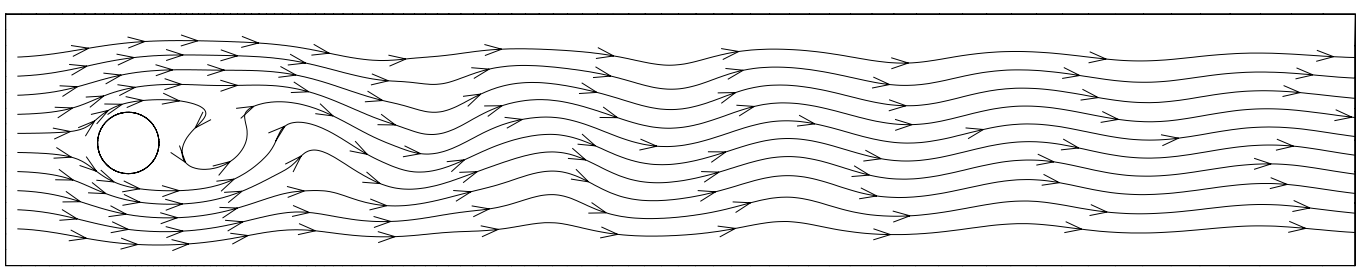

Abbildung A.7: Stromlinien der instationären Zylinderumströmung 
Bei der Validierung der Simulation einer instationären Strömung ist im Gegensatz zur stationären Strömung auch die Zeitdiskretisierung maßgeblich. Für die drei verschiedenen Gitter wurden mit unterschiedlichen Zeitschritten Rechnungen durchgeführt und die Ergebnisse in Tabelle A.3 zusammengestellt.

Tabelle A.3:Instationärer Fall 2D-2

\begin{tabular}{|l|l|l|l|l|}
\hline Gitterpunkte & $\begin{array}{l}\text { Zeitschritte } \\
\text { pro Periode }\end{array}$ & $\mathrm{c}_{\text {Dmax }}$ & $\mathrm{c}_{\text {Lmax }}$ & $\mathrm{St}$ \\
\hline \hline 27162 & 17 & $3.407>$ & $1.892>$ & $0.1961<$ \\
\hline 27162 & 612 & 3.2330 & $1.0153>$ & $0.3139>$ \\
\hline 27162 & 1225 & 3.2348 & $1.0200>$ & $0.3139>$ \\
\hline 7502 & 128 & $3.2699>$ & $1.1789>$ & $0.2609<$ \\
\hline 7502 & 301 & $3.2603>$ & $1.1107>$ & $0.2765<$ \\
\hline 7502 & 525 & $3.2611>$ & $1.1125>$ & $0.3174>$ \\
\hline 2390 & 134 & $3.3988>$ & $1.3494>$ & $0.2664<$ \\
\hline 2390 & 235 & $3.3913>$ & $1.3081>$ & $0.2842<$ \\
\hline 2390 & 459 & $3.3876>$ & $1.2875>$ & $0.2725<$ \\
\hline \hline Untere Grenze & & 3.2200 & 0.9900 & 0.2950 \\
\hline Obere Grenze & & 3.2400 & 1.0100 & 0.3050 \\
\hline
\end{tabular}

Auch hier ist, wie bereits beim stationären Testfall, eine Verbesserung der Ergebnisse mit dem Übergang vom groben zum feinen Gitter feststellbar. Ist selbst bei ausreichender räumlicher Quantisierung der Zeitschritt zu groß, z.B. 17 Zeitschritte pro Periode auf dem feinsten Gitter, so können die Resultate schlechter sein, als bei akzeptabler zeitlicher Auflösung auf gröberen Rechengittern, z.B. 128 Zeitschritte pro Periode auf dem Gitter mittlerer Feinheit.

Entgegen den Ergebnissen des stationären Testfalls liegen die Vergleichsgrößen der zeitabhängigen Simulation nicht alle genau in den vorgegebenen Grenzen. Dies liegt mit daran, dass die derzeitigen Version des $\tau^{\mathrm{i}}$-Codes eine zeitliche Diskretisierung von nur erster Ordnung zuläßt. Die größten Abweichungen weist ein Vergleich der Strouhalzahl auf. Diese sind mit ca. 3\% jedoch nicht größer als Messfehler im Experiment und in jedem Fall für die hier untersuchte Fragestellung akzeptabel. 


\section{A. Anhang}

Neben einer dem Problem angepassten örtlichen Auflösung relevanter Strömungseigenschaften ist bei der Simulation einer instationären Strömung die Wahl des Zeitschrittes ebenso bedeutend. Um zuverlässige Ergebnisse zu erhalten ist daher auf eine ausgewogenen Diskretisierung des Simulationsgebietes und der relevanten Zeitskalen $\mathrm{zu}$ achten. 


\section{Danksagung}

Diese Arbeit wurde von Herrn Prof. Dr. D. Ronneberger betreut. Bei ihm bedanke ich mich für die intensiven Diskussionen und zahlreichen Hinweise, die zum Gelingen beigetragen haben.

Das verwendete Simulationsprogramm, der $\tau^{\mathrm{i}}$-Code, wurde von Herrn Dr. R. Kessler zur Verfügung gestellt. Ihm danke ich ganz besonders u.a. für die vielen lehrreichen Diskussionen über die numerischen Aspekte dieser Arbeit und seine große Hilfsbereitschaft in Fragen der Anwendung des Codes.

Die Arbeit wurde am Institut für Strömungsmechanik des Deutschen Zentrums für Luft- und Raumfahrt e. V. in Göttingen in der Abteilung Messverfahren und Strömungsanalyse durchgeführt. Ihrem Leiter, Herrn Dr. K.-A. Bütefisch, danke ich für seine Förderung, die Nutzung u.a. der Computerressourcen und sein Interesse am Fortschritt dieser Arbeit und ihren Ergebnissen.

Prof. Dr. H. Eckelmann danke ich für die Übernahme des Korreferates und seine hilfreichen Diskussionsbeiträge u.a. im Seminar.

Ebenso danke ich allen Kollegen für die ein oder andere Hilfe bei der Realisierung dieser Arbeit. Besonders zu nennen sind hier meine „Berater und Mitstreiter“ Witold, Stefan, Thorsten und Andreas.

Das Graduierten-Kolleg „Strömungsinstabilitäten und Turbulenz“ ermöglichte mir die Verbreiterung meiner wissenschaftlichen Basis. Seinen Veranstaltungen entsprang manche Anregung. Allen Beteiligten sei hier gedankt.

Meinen Eltern danke ich, dass sie mir das Studium ermöglicht haben und mich stets mit Interesse und Anteilnahme auf meinem Weg begleitet haben.

Und Birte danke ich für ihr Verständnis und ihre Unterstützung, besonders in der Endphase der Promotion. 


\section{Lebenslauf}

10. September 1969

$1976-1980$

$1980-1989$

$06 / 89-08 / 90$

$10 / 90-04 / 93$

$04 / 93-11 / 95$

$11 / 95-11 / 96$

$02 / 96-12 / 96$

seit $12 / 96$ geboren in Kleve, deutsche Staatsangehörigkeit

Christus-König-Grundschule Kleve

Johanna-Sebus-Gymnasium Kleve

Abschluß: Allgem. Hochschulreife

Grundwehrdienst

Westfälische Wilhelms-Universität Münster

Studium der Physik

10/92 Diplomvorprüfung

Georg-August-Universität Göttingen

Fortsetzung des Physikstudiums

11/ 95 Diplomhauptprüfung

04/94 - 09/95 Anfertigung der Diplomarbeit am Institut für Medizinische Physik und Biophysik

Gastwissenschaftler am Institut für Medizinische Physik und Biophysik

wissenschaftliche/studentische Hilfskraft bei der Gesellschaft für wissenschaftliche Datenverarbeitung $\mathrm{mbH}$ Göttingen

Beschäftigung beim Deutschen Zentrum für Luft- und Raumfahrt e.V., Göttingen; Mitglied im GraduiertenKolleg „Strömungsinstabilitäten und Turbulenz“ 